\title{
AN OVERVIEW OF THE OLD WORLD SPECIES OF PSEUDOCOLLINELLA DUDA (DIPTERA: SPHAEROCERIDAE) WITH DESCRIPTION OF A NEW SUBGENUS
}

\author{
LÁSZLó PAPP \\ H-1182 Budapest, Beremend u. 43, Hungary \\ E-mail:flyer.papp@gmail.com
}

\begin{abstract}
A new subgenus of Pseudocollinella Duda, Setiopacifrons subgen. n. (type species P. (Setiopacifrons) dupliciseta (Duda, 1925)) is described with five new species from the Afrotropical region (P. (S.) congoana sp. n., $P$. (S.) normalis sp. n., $P$. (S.) prima sp. n., $P$. (S.) setisternalis sp. n. and $P$. (S.) vulnerata sp. n.) and 11 new species from other Old World regions (P. (S.) communis sp. n. (Thailand), $P$. (S.) formosensis sp. n. (Taiwan), $P$. (S.) japonica sp. n., $P$. (S.) koreana sp. n., $P$. (S.) paradupliciseta sp. n. (Taiwan), P. (S.) pilitibia sp. n. (Thailand), P. (S.) setipuga sp. n. (Thailand), $P$. (S.) simplicisternum sp. n. (Taiwan), P. (S.) tercia sp. n. (Thailand), $P$. (S.) trifida sp. n. (Taiwan) and P. (S.) vietnamensis sp. n.). P. (Pseudocollinella) marshalli sp. n. and P. (P.) mongolica sp. n. are described from Mongolia. Spinotarsella Richards, 1929 has been reinstated as a subgenus of Pseudocollinella Duda, 1924 with the description of $P$. (Spinotarsella) pseudohumida sp. $\mathbf{n}$. from the P. R. of China. Keys to the subgenera and to the species of Setiopacifrons are given. With 229 original figures.
\end{abstract}

\section{INTRODUCTION}

Pseudocollinella Duda, 1924 is a species rich genus of the subfamily Limosininae. The former "old" (wider) concept of the genus Leptocera Olivier, 1913 was given up by the sphaerocerid taxonomist beginning with the 1990's years. Although one can give a number of features, which characterise the wider concept (mid tibia with distinct ventral preapical seta, ventroapical seta usually absent (except for Pteremis); when ventral preapical missing (i.e. mid tibia without any longer seta ventrally), then mid basitarsus ventrally with a distinct seta, 2 frontoorbital, ocellar, outer and inner vertical, outer and inner occipital setal pairs are strong, postocellar small (or even missing), hypandrium fused to epandrium.

All the evidences found hitherto corroborate that the Leptocera + Rachispo$d a$ clade is obviously monophyletic. But inclusion of Opacifrons Duda into this clade is biased (see more in Marshall \& Smith 1993, Marshall \& LangstafF 1998). Although the characterisation of Opacifrons had been made correctly as early as Roháčé (1975) - works like Marshall and Langstaff (1998), RонÁčеK (1998), PAPP (2012), etc., only corroborated Roháček's concept. As for the severe distinction of Opacifrons Duda and Pseudocollinella Duda, that was not established for a period of time. MARSHALL and SMITH (1993) characterised correctly that genus, based on the species in the Nearctic region (numerous ones were described as new), with inclusion of Spinotarsella Richards, 1929. 
PAPP, L.

This last action was made without maintaining even the subgeneric status of Spinotarsella. A number of the formerly described species of the Leptocera complex were correctly included in Pseudocollinella (see below). For some of the species relegated to Opacifrons, they expressed their bias.

This project was initiated by our work for the Afrotropical manual (PAPP \& RонÁ ̌́Ек 2016), since it became clear that there was no former record of the genus Pseudocollinella Duda from the Afrotropical region.

\section{MATERIAL AND METHODS}

This paper is based on studies of many double mounted (mostly minuten pinned) specimens of Pseudocollinella, which are preserved in the Diptera Collection of the Hungarian Natural History Museum, Budapest (below: HNHM), in Institute royal des Sciences naturelles de Belgique, Brussels (below: IRSNB) and in the Entomology Department, National Museum, Bloemfontein, South Africa (below: BMSA). In the case of type specimens these abbreviations are given in every case. A limited number of specimens from other collections are specifically mentioned.

Abdomina of several (at least one) specimens of each species were removed (after softening them in a closed water vapour glass) and treated with sodium-hydroxide and lactic acid, washed, etc. on the standard way; those abdomina with genitalia are kept in polyethylene microvial with glycerol. I have to note that the abdomina of the Pseudocollinella species are connected to the metathorax on a very broad surface. As a consequence, removing abdomina is difficult and it occurs frequently, that the abdomen comes with a part of metathorax or including also hind leg(s). In the latter cases also those parts are kept in the microvial. In cases a short "gen. prep." is given when the abdomina with genitalia are prepared and preserved in a plastic microvial with glycerol (on the pin of the specimen).

The figures were made under a normal 1/1 cover glass put over some glycerol on a slightly hollowed microscopic slide. The genitalia were positioned under an OLYMPUS SZ-ST stereomicroscope, usually under 100× magnification; figures were made on an OLYMPUS BX40 microscope with an OLYMPUS U-DA device. The figures were made almost exclusively under a $667 \times$ magnification.

Measurement units are not used in this paper but all measurements are given in $\mathrm{mm}$. Length of some of the abdomina were measured after preparation.

In the records below, label data are quoted letter by letter; hand-written label data are given in quotation marks, whereas supplementary letters and my annotations of label data are in square brackets. Data on subsequent labels are separated by a -.

\section{TAXONOMY}

\section{Genus Pseudocollinella Duda, 1924}

Type species: Limosina septentrionalis Stenhammar, 1855 (mon).

The generic features of Pseudocollinella have been summarised by MARSHALl and SMith (1993) and RoháčEK (1998). Here we show those of the subgenera and of the genus Opacifons in the following table: 


\begin{tabular}{|c|c|c|c|c|}
\hline & Opacifrons & Pseudocollinella & Spinotarsella & Setiopacifrons \\
\hline $\begin{array}{l}\text { Inner orbital } \\
\text { setae }\end{array}$ & no & mostly no & $\begin{array}{c}2+\text { several } \\
\text { short }\end{array}$ & $\begin{array}{l}2 \text { strong (or }+ \\
\text { several short) }\end{array}$ \\
\hline $\begin{array}{l}\text { Posterior } p d \text { seta } \\
\text { on } t 2\end{array}$ & no & yes & yes & yes \\
\hline $\begin{array}{l}\text { Dorsocentral } \\
\text { setae }\end{array}$ & $\begin{array}{l}0+2(3) \text {, ant. } \\
\text { short }\end{array}$ & $1+2$ & $1+2$ & $\begin{array}{c}0+2, \text { or }+1 \text { small } \\
\text { presut }\end{array}$ \\
\hline Acrostichal setae & 8 rows & 6 rows & 6 rows & $6-8$ rows \\
\hline $\begin{array}{l}\text { Hypandrial } \\
\text { apodeme }\end{array}$ & no & yes (fused) & long, separate & $\begin{array}{c}\text { no or yes } \\
\text { (fused) }\end{array}$ \\
\hline Postgonite(s) & $\begin{array}{l}\text { simple, no } \\
\text { setulae }\end{array}$ & $\begin{array}{l}\text { simple, longish, } \\
\text { with/without } \\
\text { setulae }\end{array}$ & $\begin{array}{l}\text { simple, with- } \\
\text { out setulae }\end{array}$ & $\begin{array}{l}\text { bipartite or } \\
\text { simple }\end{array}$ \\
\hline Surstylus & single pair & ant. + post. sur & ant. + post. sur & ant. + post. sur \\
\hline Male sternite 5 & $\begin{array}{l}\text { rather simple } \\
\text { with/without } \\
\text { caudal process }\end{array}$ & $\begin{array}{c}\text { mostly with } \\
\text { paired caudal } \\
\text { processes/lobes }\end{array}$ & simple & various \\
\hline $\begin{array}{l}\text { Female epiproct } \\
\text { and cerci }\end{array}$ & separated & separated & separated & fused \\
\hline
\end{tabular}

Remark. Although it is a generally accepted convention that in the family Sphaeroceridae we do not speak about two pairs of surstyli, in males of $P_{s e u-}$ docollinella surstylus is divided into two. For short, I do not name the anterior one as "anterior part of surstylus", but mostly simply anterior surstylus, and, posterior surstylus, as regards the posterior part.

A key for the quick identification of the subgenera is given here.

\section{Key to the subgenera of Pseudocollinella Duda}

1. Mostly no inner orbital setae. A pair of strong presutural dorsocentral setae is always present. Subepandrial sclerite with a strong ventral process. Female epiproct and cerci separated.

Pseudocollinella Duda

- Always 2 inner orbital pairs (or even additional short ior setae) present. Presutural dorsocentral setae lacking or rather weak.

2. A pair of strong presutural acrostichal setae present. Presutural $d c$ pair present. Male hypandrial apodeme large, separate from arms. Subepandrial sclerite with a strong ventral process. Distiphallus very short, basiphallus very short, forming a ring. Female epiproct and cerci separated.

Spinotarsella Richards 
- No strong presutural acrostichal setae. Presutural $d c$ pair mostly lacking. Male with or without hypandrial apodeme, if yes, fused to arms. Subepandrial sclerite without a long ventral process. Distiphallus large and structured, basiphallus usually longer than broad with a distinct epiphallus. Female epiproct and cerci fused. Setiopacifrons subgen. $\mathrm{n}$.

\section{Subgenus Pseudocollinella Duda}

Some of the features, which define Pseudocollinella s.str., are summarised below:

Male sternite 5 with a pair of hairy lobes medio-caudally; the paired lamella of subepandrial sclerite joins to the dorsal edge of cerci (cf. Marshall \& SмITH 1993), this lamella curving anteriorly and inside forms a nearly parallel plate with cerci; subepandrial sclerite (sternite 10 of MARshall \& SMITH 1993) with a pair of long ventral processes which represent a third genital forceps driven surstylar pair apart; however, that long ventral forceps do not join either to epandrium or surstyli; caudally to this there is a pair of narrow lobes, which connected to pseudocerci. Epandrial lobe (e.g. figs 53-54 of MARshall \& SMITH 1993) overhanging posterior part of surstylus, anterior surstylus bilobed, posterior surstylus elongate and lacking the large setae that characterise closely related genera. Male sternite 5 and female sternite 8 are similar throughout the genus.

The only common European species of Pseudocollinella s.str., P. septentrionalis (Stenhammar, 1855) as well as the two new species described below from Mongolia belong to the P. septentrionalis group of Marshall \& SMith (1993). There are two known Nearctic species in this group: P. aequilifrons Marshall in Marshall \& Smith, 1993 and P. caelobata (Spuler, 1924). The most characteristic (mostly monophyletic) features of the group are as follow: arista subequal the height of head; 3 or more rows of genal setae; epandrial lobe not fused with epandrium; subepandrial sclerite elongate with inner fringe; male sternite 5 with posteromedial lobes; anterior surstylus bulbous, basiphallus elongate; postgonite setulose posteriorly.

\section{Pseudocollinella septentrionalis (Stenhammar, 1855)}

(Figs 17-24, 29, 31-33)

Material studied (HNHM): Bulgaria: 1 male: “Mussalla 929. VIII. 30. 2600 [?m] - Bulgaria Szilády; 1 female: Mt. Balkan 928. Szilády, VII. 11. - “Gyumruk esul 2000-2300 m”. Hungary: 1 female: Mecsek-hegys., “Zobák. [illeg.] “ [Komló, Zobákpuszta]- “1964. VI. 23." Dr. Wéber.

The specimen from the Magadan obl., Semichanka (PAPP 1977) is in all probability not conspecific with P. septentrionalis. 
Through the courtesy of Dr. J. Roháček (Opava, Czech Republic) several years ago I studied a female from Denmark (Dania: Sjaelland, Furesø, shore, 8. 7. 1969, M. Chvála - "Opacifrons septentrionalis (Stenh.) \&", J. Roháček det. 1969 - "Pseudocollinella septentrionalis (Stenh.) + " J. Roháček det. 2001). That time this specimen was very important for me in order to have a correct characterisation of this species. I would quote here my notices, as follow:

Body length $3.02 \mathrm{~mm}$, wing length $2.67 \mathrm{~mm}$, wing width $1.10 \mathrm{~mm}$. Frons pink anteriorly and between interfrontal and orbital plates. On the right side 4, on the left side 5 strong interfrontal setae. 5 colourless setae on parafacialia. 4 rows of acrostichals behind suture, 6 rows anteriorly. Longest of the 3 anterior setae of mid tibia $0.13 \mathrm{~mm}$. Mid tibia with preapical seta $0.23 \mathrm{~mm}$, mid basitarsal ventral seta $0.013 \mathrm{~mm}$ long.

Male sternite 5 (Fig. 17) shorter than that of both Mongolian species, medio-caudal part with a sagittal incision, marginal row of short setulae shorter and oblique (not transverse as in the Mongolian species). Synsternite (Fig. 18) with very broad (lengthened to the right) sternite 6 part, sternite 7 part less broad, sternite 8 part without darker ribs on its ventral surface. Ventral appendix of the subepandrial sclerite (Figs 21-22) slightly curved with thin setulae on all along medial margin (some of them are also on lateral margin). Anterior (medial) lobe of anterior surstylus (Fig. 20) comparatively small, caudal lobe seems to be "cut" apically. Apical half of posterior surstylus (Fig. 19) long and thin. Apical half of postgonite (Fig. 23) shorter and comparatively rather thick. Apical part of distiphallus (Fig. 24) long and strongly dorsally curved.

Female sternite 7 (Fig. 31) with a deep groove medio-caudally, which filled up by less sclerotised membrane covered by fine hairs; caudal half of the sternite with numerous medium-long setae. Medially directed process of tergite 8 (Fig. 29) more oblique than in $P$. mongolica, its latero-caudal setal pair is rather long. Epiproct (tergite 9, Fig. 32) with a pair of small hairy caudal processes, its dorsal pair of setae very long. Spermathecae (Fig. 33) ovoid in shape but surface with small swellings, so spermathecae are similar to the fruit of Morus trees.

The differentiating features of $P$. septentrionalis and those of the two new species from Mongolia are given in the key below.

\section{Pseudocollinella (P.) marshalli sp. n.}

(Figs 1-8, 25-28)

Leptocera (Opacifrons) septentrionalis: L.Papp 1973: 417-418, misidentification.

Holotype male (HNHM): Mongolia: Uvs aimak, am Fluss Baruun-turuun gol neben Somon Baruun-turuun, 1280 m, Exp. Dr. Z. Kaszab, 1968 - Nr. 1012, 25. VI. 1968.

Paratypes (HNHM): 6 males 7 females: same as for the holotype; 1 female: ibid., Chövsgöl aimak, Alag Mort, $42 \mathrm{~km}$ NO von Pass Chaldzan Sogotyn davaa, am Fluss Tesijngol, 1900 m - Nr. 1110, 14. VII. 1968; 1 male (Univ. Guelph Insect Collection): [Russia]: Siberia, Altai region, Teletskoya Lk; shore sweeps, 15-20. vii. 1991, S. A. Marshall. 


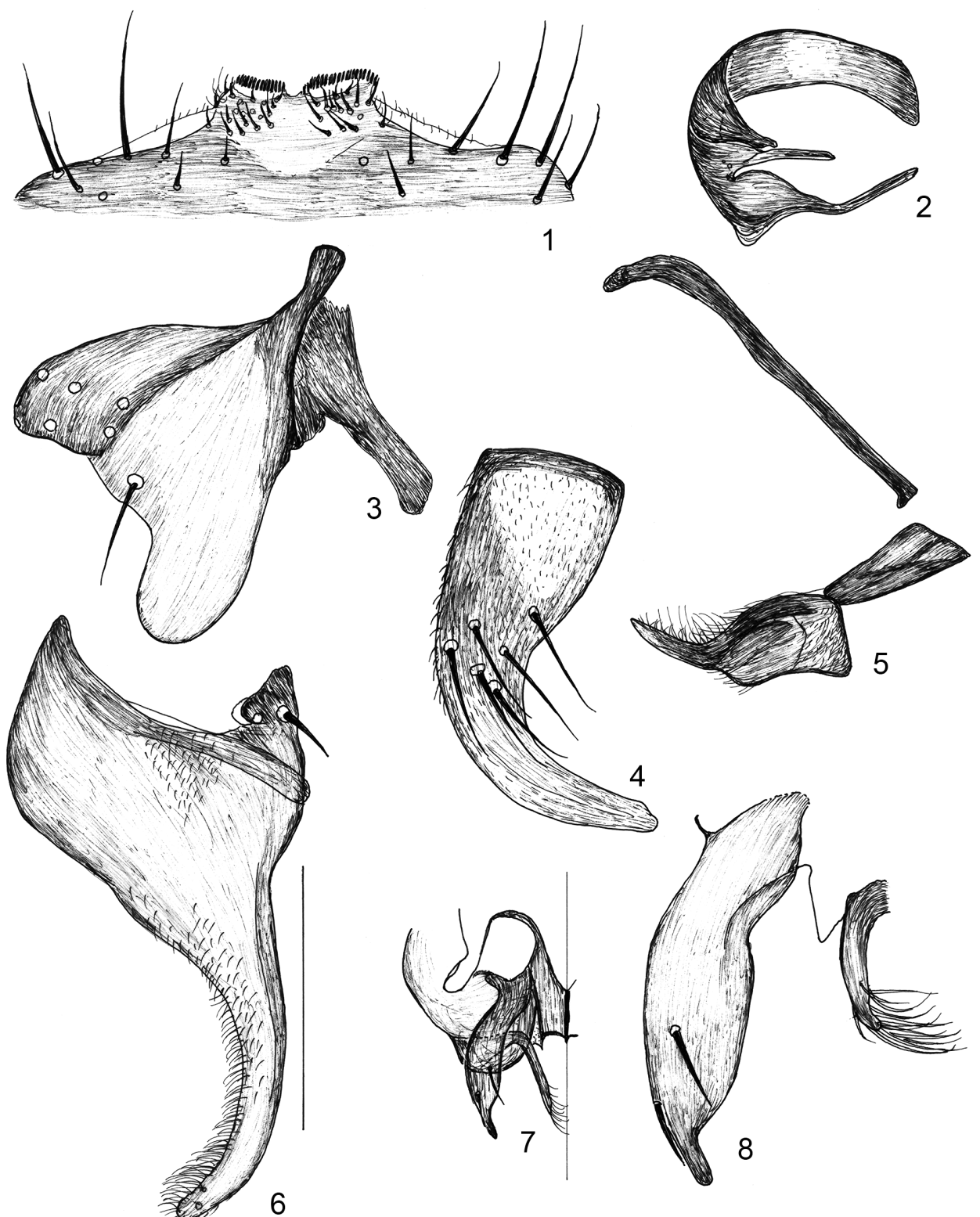

Figs 1-8. Pseudocollinella marshalli sp.n., male sternite 5 and genitalia: $1=$ sternite 5 , ventral view, 2 = synsternite, ventral-subventral view, 3 = anterior surstylus, broadest (ventralsubventral) view, 4 = posterior surstylus, broadest (sublateral-subcaudal) view, 5 = phallus and phallapodeme, lateral view, 6 = postgonite, broadest extension, sublateral, slightly subanterior view, 7 = subepandrial sclerite, its appendix and posterior surstylus, anterior view; $8=$ posterior surstylus and appendix of the subepandrial process, broadest (sublateral) view. Scale: $0.4 \mathrm{~mm}$ for Fig. 2, $0.2 \mathrm{~mm}$ for Figs 1, 5, 7, $0.1 \mathrm{~mm}$ for Figs 3-4, 6, 8 
Measurements in mm: body length 2.50 (holotype), 2.34-2.73 (paratypes), wing length 2.92 (holotype), 2.74-3.10 (paratypes), wing width 1.13 (holotype), 1.06-1.18 (paratypes).

Medio-caudal part of male sternite 5 (Fig. 1) in the groove of the anterior larger part of the sternite less sclerotised with comparatively long setae. There are well ordered evenly short thick setulae on the margin. Synsternite (Fig. 2) with narrow sternite 6 part, sternite 7 part shorter than in mongolica, sternite 8 without ribs on vental surface. Subepandrial sclerite (Figs 7-8) small, setae on its ventral appendix concentrated to its apex. Anterior surstylus (Fig. 3) with comparatively narrower anterior (medial) lobe and larger caudal lobe. Caudally curved apical half of posterior surstylus (Fig. 4) thicker than in its congeners. Apical half of postgonite (Fig. 6) narrow and evenly curved. Apical part of distiphallus (Fig. 5) thinner and less curved than in P. mongolica.

Female sternite 7 (Fig. 25) with a broad medio-caudal emargination; long setae on the caudal half of the sternite. Tergite 8 (Fig. 28) with long and thin medial process, its caudal setal pair is not particularly long. Anterior part of sternite 8 (Fig. 28) weakly sclerotised, caudally with 2 pair of thick setae. Dorsal subbasal pair of setae on epiproct (Fig. 26) medium-long. Spermathecae (Fig. 27) ovoid with fine transverse sculpture.

Etymology. This new species has been dedicated to Prof. Dr. Stephen A. Marshall (Guelph University) in recognition of his great contribution to the taxonomy of the Sphaeroceridae.

P. marshalli sp. $\mathrm{n}$. is member of the septentrionalis species-group of the subgenus. The differentiating features to the related Palaearctic species are given in the key below.

In the key of the Nearctic species (Marshall \& SMith 1993) it keys to couplet 8 but there is a conflict between aequilifrons and calobata; details of genital parts of the new species are different from both of the Nearctic species.

\section{Pseudocollinella (P.) mongolica sp. n.}

$$
\text { (Figs 9-16, 30, 34-36) }
$$

Leptocera (Opacifrons) septentrionalis: L. Papp 1973: 417-418, misidentification.

Holotype male (HNHM): Mongolia: Bajan Ölgij aimak, im Tal des Flusses Chavcalyn gol, 25 km O von Somon Cagannuur, 1850 m, Exp. Dr. Z. Kaszab, 1968 - Nr. 1056-7, 3. VII. 1968.

Paratypes (HNHM): 4 males 7 females: same as for the holotype (plus a seriously damaged $\delta$, not designated as a paratype); 8 males 16 females: ibid., Uvs aimak: am Fluss Baruun-turuun gol neben Somon Baruun-turuun, 1280 m - Nr. 1012, 25. VI. 1968; 1 male: ibid., Chovd aimak: am Fluss Bulgan gol, cca. 15 km N von Somon Bulgan, 1300 m - Nr. 640, 7. VII. 1966; 1 male: ibid., Bajanchongor aimak, Changaj Gebirge, Somon Zag am Fluss Zag gol, 2100 m - Nr. 709, 18. VII. 1966; 1 male: Gobi Altaj aimak, Zachuj Gobi, 10 km von Chatan Chajrchan Gebirge, 1150 m - Nr. 594, 27. VI. 1966.

Measurements in mm: body length 2.45 (holotype), 2.30-2.75 (paratypes), wing length 2.98 (holotype), 2.73-3.11 (paratypes), wing width 1.14 (holotype), 1.08-1.20 (paratypes).

Body features as in P. septentrionalis. Medio-caudal part of male sternite 5 (Fig. 9) with shorter setae than in marshalli (at least setae are thinner there, well ordered evenly short thick setulae emerge on the margin). Anterior medial part of the sternite is less mela- 
PAPP, L.

nised. Synsternite (Fig. 10) with a distinct right-side sclerite, sternite 7 part reaching farther on the right side, ventral surface of sternite 8 part with darker ribs. Subepandrial sclerite (Figs 15-16) slightly larger than in marshalli, thin setae on its ventral appendix are along its medial margin (i.e. not concentrated on its apical part). Anterior surstylus (Fig. 11) with broader anterior (medial) lobe and comparatively smaller caudal lobe, which bears thick setae. Apical (recurved) half of posterior surstylus thinner than in P. marshalli. Apical half of postgonite (Fig. 14) thicker and angularly bent. Apical part of distiphallus (Fig. 13) thicker and more dorsally curved.

Female sternite 7 (Fig. 34) subquadratic, medio-caudal emargination not straight basally, sternite 7 with long setae on its caudal half. Tergite 8 (Fig. 30) with long and thin medial process, its caudal setal pair is very long. Anterior part of sternite 8 (Fig. 30) better sclerotised, caudally with 2 pair of short setae. Epiproct (tergite 9, Fig. 35) somewhat shorter than long, its setal pair is the shortest in this species group. Spermathecae (Fig. 36) globular rather than ovoid with fine transverse sculpture.

Etymology. The specific epithet of this new species refers to its type locality (Mongolia).

P. mongolica sp. n. is a typical member of the septentrionalis species group. The differentiating features to the related Palaearctic species are given in the key below. In possession of a good material for comparison also the females are to be identified. In the key of the Nearctic species (MARshall \& SMith 1993) it keys to P. septentrionalis (Stenhammar). Indeed, its closest relative is that species (see below).

1. Frons uniformly dark grey. Mesonotum dark grey dusted (microtomentum is dark grey). Dorsal half of mid basitarsus always dark grey. Male genitalia (Figs 1-8). In female genitalia (Figs 25-28) spermathecae are ovoid with fine transverse sculpture (Fig. 27). $\quad$ P. marshalli sp. n.

- $\quad$ Anterior 1/4-1/3 of frons, facial plate, parafacialia and parts of gena yellowish or reddish. Dorsal half of mid basitarsus not seldom yellowish. 2

2. Mesonotum subshiny, microtomentum darker grey than in P. mongolica. Cilia on apex of first flagellomere at least $0.01 \mathrm{~mm}$. In the male genitalia (Figs 17-24) anterior (medial) lobe of anterior surstylus (Fig. 20) comparatively small, caudal lobe seems to be "cut" apically. In female genitalia (Figs 29, 31-33) spermathecae are ovoid in shape but surface with small swellings, so spermathecae are similar to the fruit of Morus trees.

P. septentrionalis (Stenhammar, 1855)

- Mesonotum lighter grey dusted. Cilia on apex of first flagellomere shorter than $0.01 \mathrm{~mm}$. In the male genitalia (Figs 9-16) anterior surstylus (Fig. 11) with broader anterior (medial) lobe and comparatively smaller rounded caudal lobe. In female genitalia (Figs 30, 34-36) spermathecae globular rather than ovoid with fine transverse sculpture.

P. mongolica sp. n. 


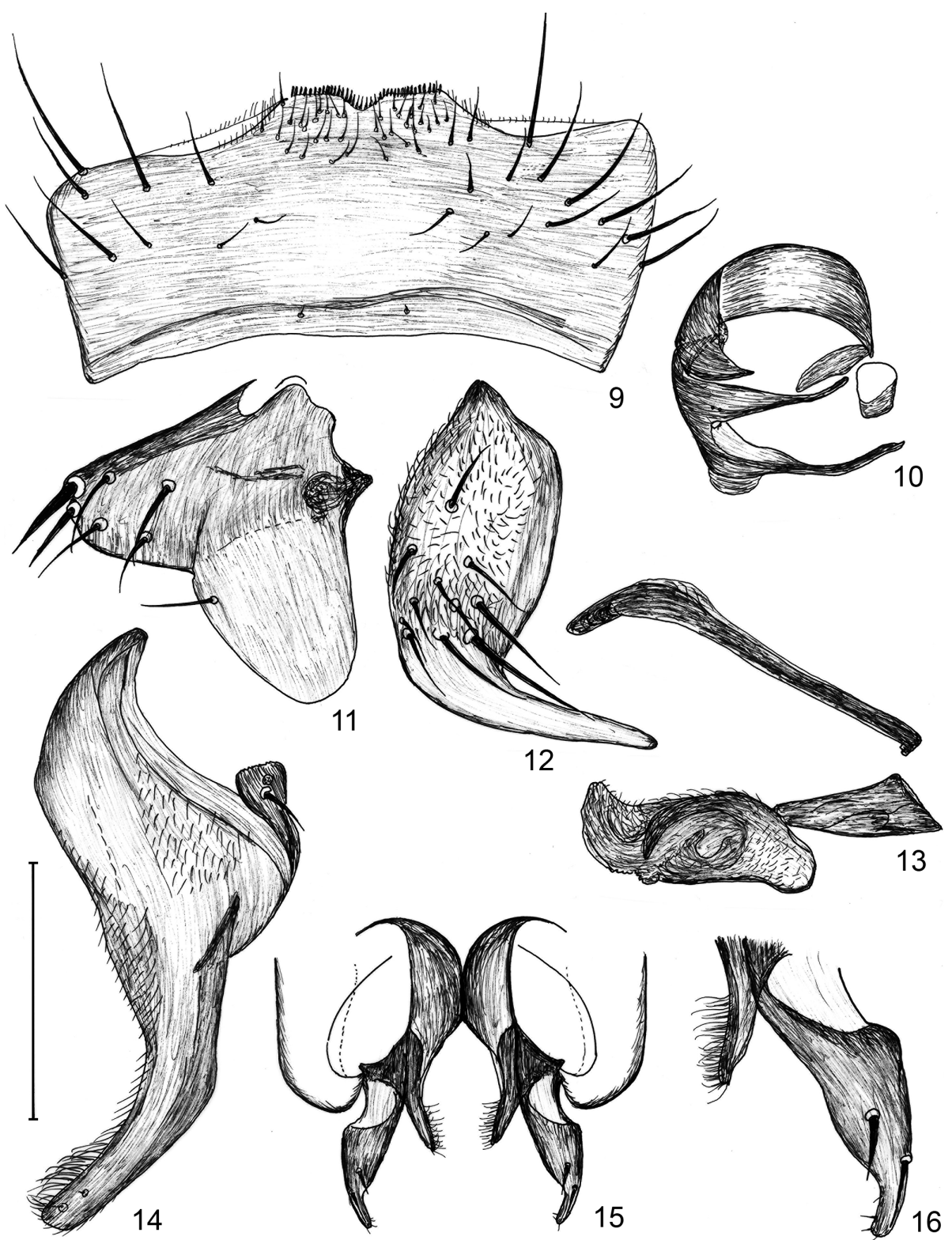

Figs 9-16. Pseudocollinella mongolica sp. n., male sternite 5 and genitalia: $9=$ sternite 5, ventral view, 10 = synsternite, ventral-subventral view, 11 = anterior surstylus, broadest (ventral-subventral) view, 12 = posterior surstylus, broadest (sublateral-subcaudal) view, $13=$ phallus and phallapodeme, lateral view, 14 = postgonite, broadest extension, sublateral, slightly subanterior view, 15 = ventral part of epandrium, subepandrial sclerite, its appendix and posterior surstylus, anterior view, $16=$ posterior surstylus and appendix of the subepandrial process, broadest (sublateral) view. Scale: $0.4 \mathrm{~mm}$ for Fig. 10, $0.2 \mathrm{~mm}$ for Figs 9, 13, 15, $0.1 \mathrm{~mm}$ for Figs 11-12, 14, 16. 


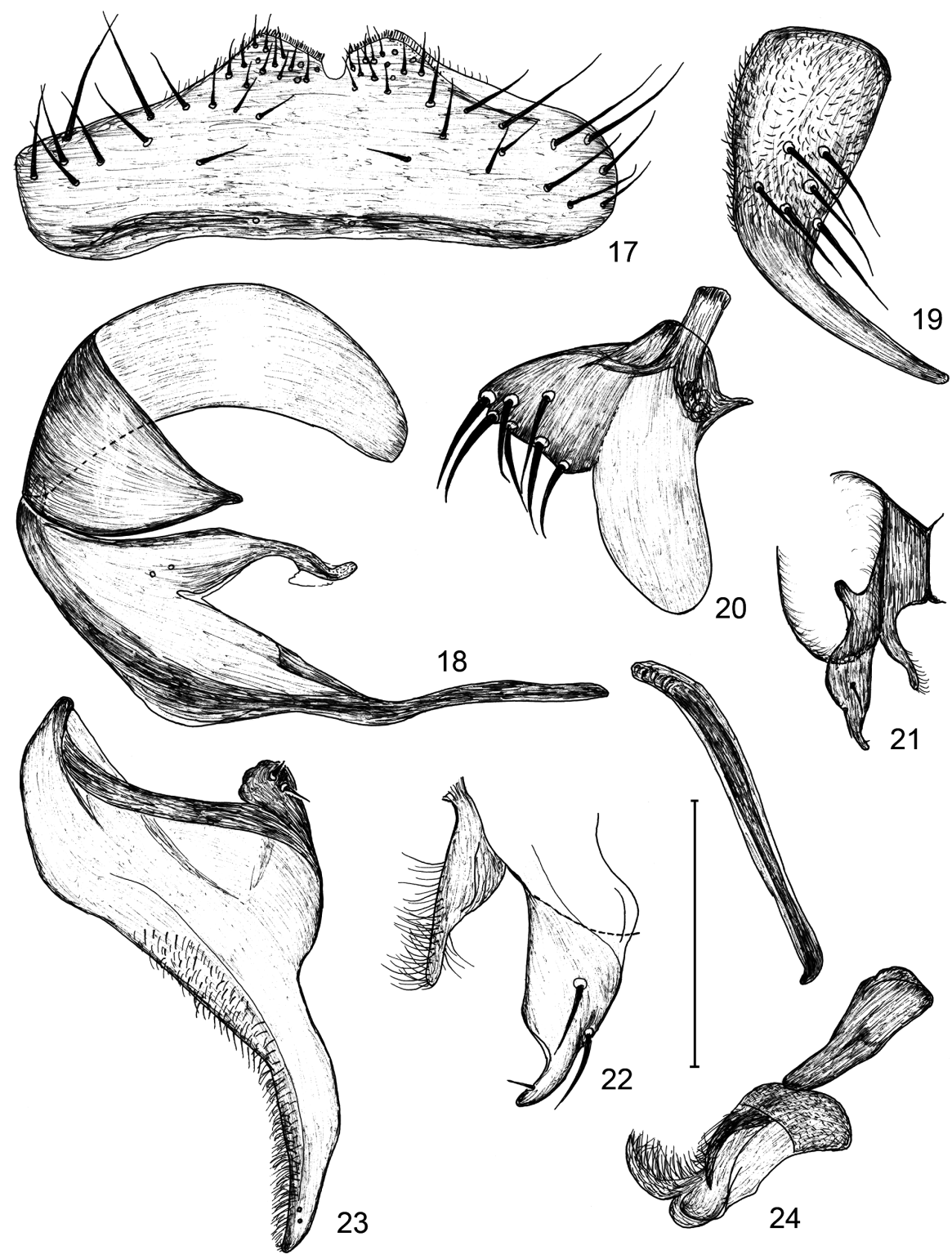

Figs 17-24. Pseudocollinella septentrionalis (Stenhammar, 1855), male sternite 5 and genitalia: 17 = sternite 5 , ventral view, 18 = synsternite, ventral view, 19 = posterior surstylus, broadest (sublateral-subcaudal) view, $20=$ anterior surstylus, broadest (ventral-subventral) view, 21 = ventral part of epandrium, subepandrial sclerite, its appendix and posterior surstylus, anterior view, 22 = posterior surstylus and appendix of the subepandrial process, broadest (sublateral) view, 23 = postgonite, broadest extension, sublateral, slightly subanterior view, 24 = phallus and phallapodeme, lateral view. Scale: $0.2 \mathrm{~mm}$ for Figs 17-18, 21, 24, $0.1 \mathrm{~mm}$ for Figs 19-20, 22-23. 


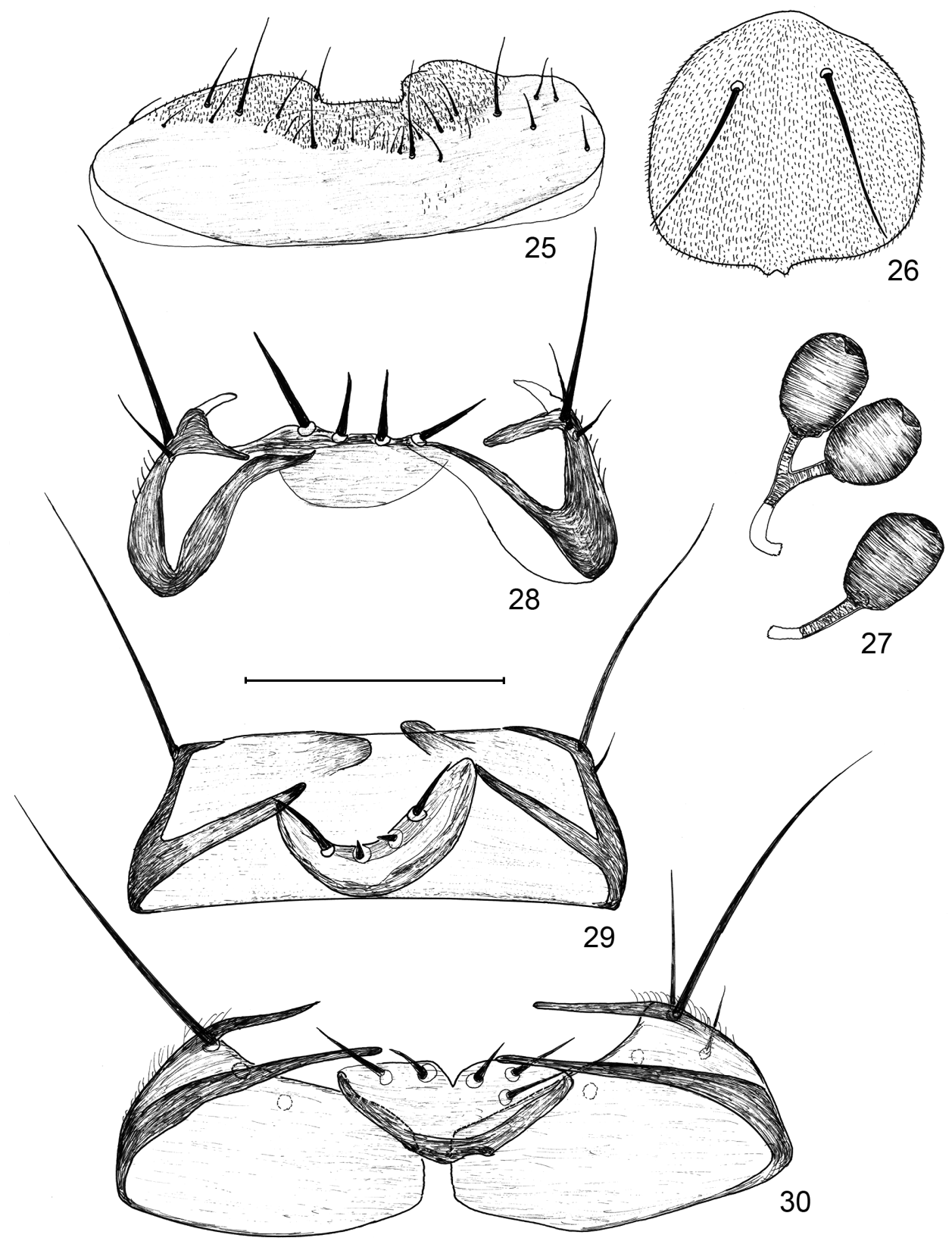

Figs 25-30. Pseudocollinella spp., female postabdomen and genitalia. 25-28= P. marshalli sp. n.: 25 = sternite 7 , ventral view. $26=$ epiproct, dorsal view, $27=$ spermathecae, $28=$ tergite and sternite 8 , ventral view; $29=P$. septentrionalis (Stenhammar, 1855), tergite and sternite 8 , ventral view; $30=P$. mongolica sp. $\mathrm{n}$., tergite and sternite 8 , ventral view. Scale: $0.2 \mathrm{~mm}$ for Fig. 25, $0.1 \mathrm{~mm}$ for Figs 26-30. 


\section{Pseudocollinella ochrea - tunisica species group}

These two species do not fit to any of the species groups (subgenera) of the genus. In lack of other evidences they have been left in the nominate subgenus.
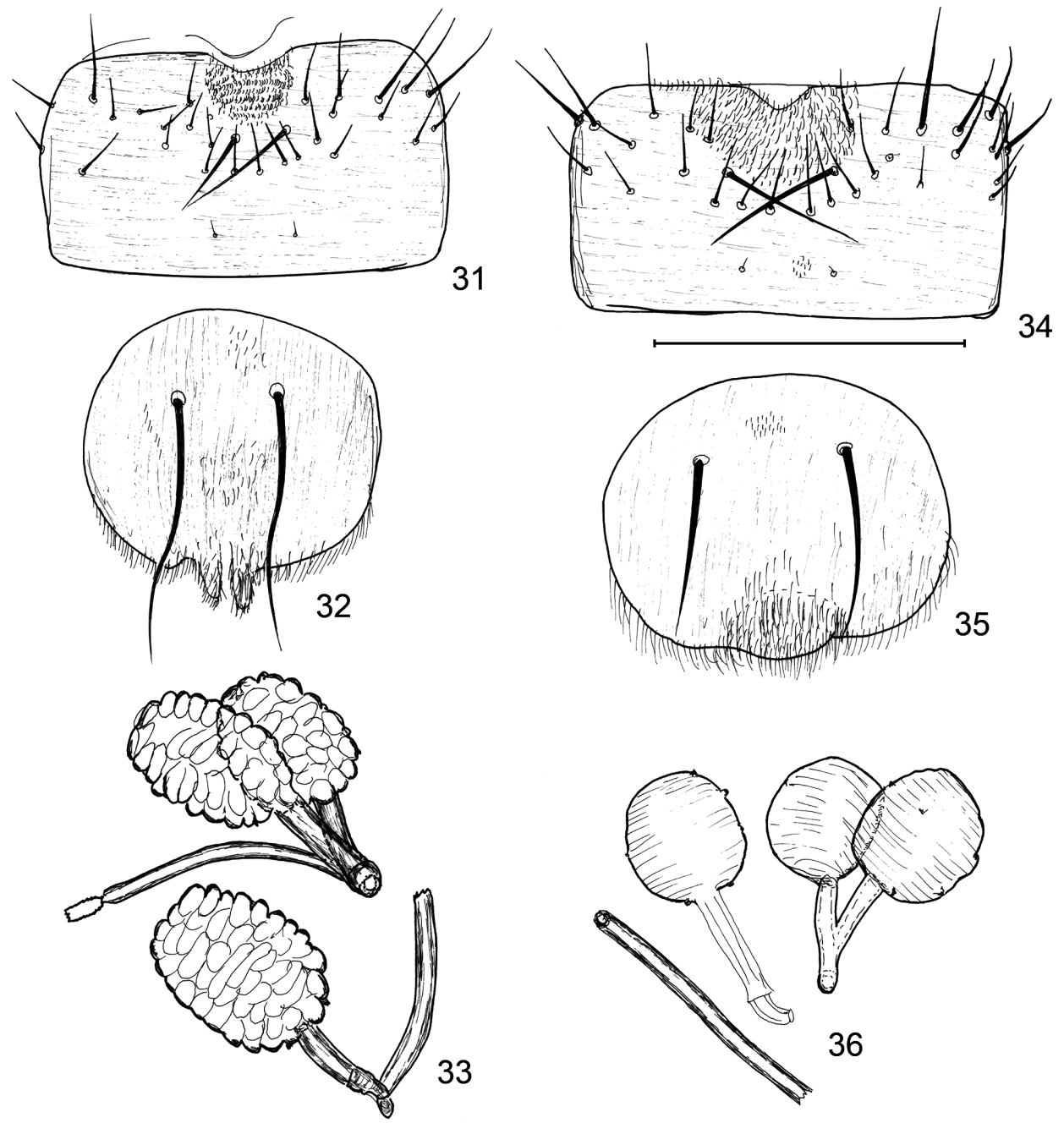

Figs 31-36. Pseudocollinella spp., female postabdomen and genitalia. 31-33 = P. septentrionalis (Stenhammar, 1855): $31=$ sternite 7 , ventral view, 32 = tergite 9, dorsal view, $33=$ spermathecae with ducts; $34-36=P$. mongolica sp. n.: $34=$ sternite 7 , ventral view, $35=$ tergite 9 , dorsal view, 36 = spermathecae with ducts. Scale: $0.2 \mathrm{~mm}$ for Figs 31, 34, $0.1 \mathrm{~mm}$ for Figs 32-33, 35-36. 
Characteristic features of the group: eyes very small, consequently gena broad, inner orbitals very short, hardly discernible (!). Postvertical pair of setae rather well developed. Mid trochanter with 2 pairs of rather long setae.

\section{Pseudocollinella ochrea (L. Papp, 1974)}

(Figs 37-39)

It was described from Mongolia (Yuzhno-Gobiyskiy aimak, bliz poselka Dund-Gol, Kerzhner, 20-21. VIII. 1969.). The holotype male and one female paratype are preserved in the Zoological Institute of the Russian Academy of Sciences, St. Petersburg (not studied now). One female was found also in Kazakhstan (PApp 1979).

Material studied: female paratype (HNHM).

Differentiating features to $P$. tunisica, other than in the original description: Costal setae minute, no longer setae on costa.

Female tergite 7 dorsally c. 3 times longer than in P. tunisica, its submedial pair of setae half as thick and $0.09 \mathrm{~mm}$ long. Sternite 6 very short, rather asymmetrical (? a morphosis, since I saw postabdomen of this female only). Dorsal part of tergite 8 short, with 3 pairs of strong setae (Fig. 37). Ventral parts of tergite 8 rather similar (with 2 pairs of processes) but sternite 8 differently shaped. Apical part of cercus (Fig. 38) with 4 short and 1 medium-long setae. Epiproct membranous with a short pair of setae (Fig. 37). Hypoproct with 2 pairs of thicker setae only (Fig. 38), which are less thick than in $P$. tunisica. Paired spermathecae: ducts much longer but there is no sclerotised common duct (Fig. 39); spermathecae, incl. unpaired one, are with somewhat stronger lateral sclerotisation, basally with fine sharp processes.

\section{Pseudocollinella tunisica (L. Papp, 1977)}

(Figs 40-43)

Material studied: female holotype (HNHM), in a Canada balsam preparation between 2 small pieces of cover glass.

Male unknown.

Female tergite 8 (Fig. 42) with 2 pairs of long thin processes (best seen in a subcaudal view). Sternite 8 (Fig. 41) not wholly symmetrical, bordered by membranous "cover". Paired spermathecae (Fig. 40) with sclerotised common duct, individual ducts shorter. Spermathecae, incl. unpaired spermatheca (Fig. 43), globular with sparse transverse lines, their basal part conical with fine sharp processes.

RoнáčEK et al. (2001) correctly placed the two species in the genus Pseudocollinella. 

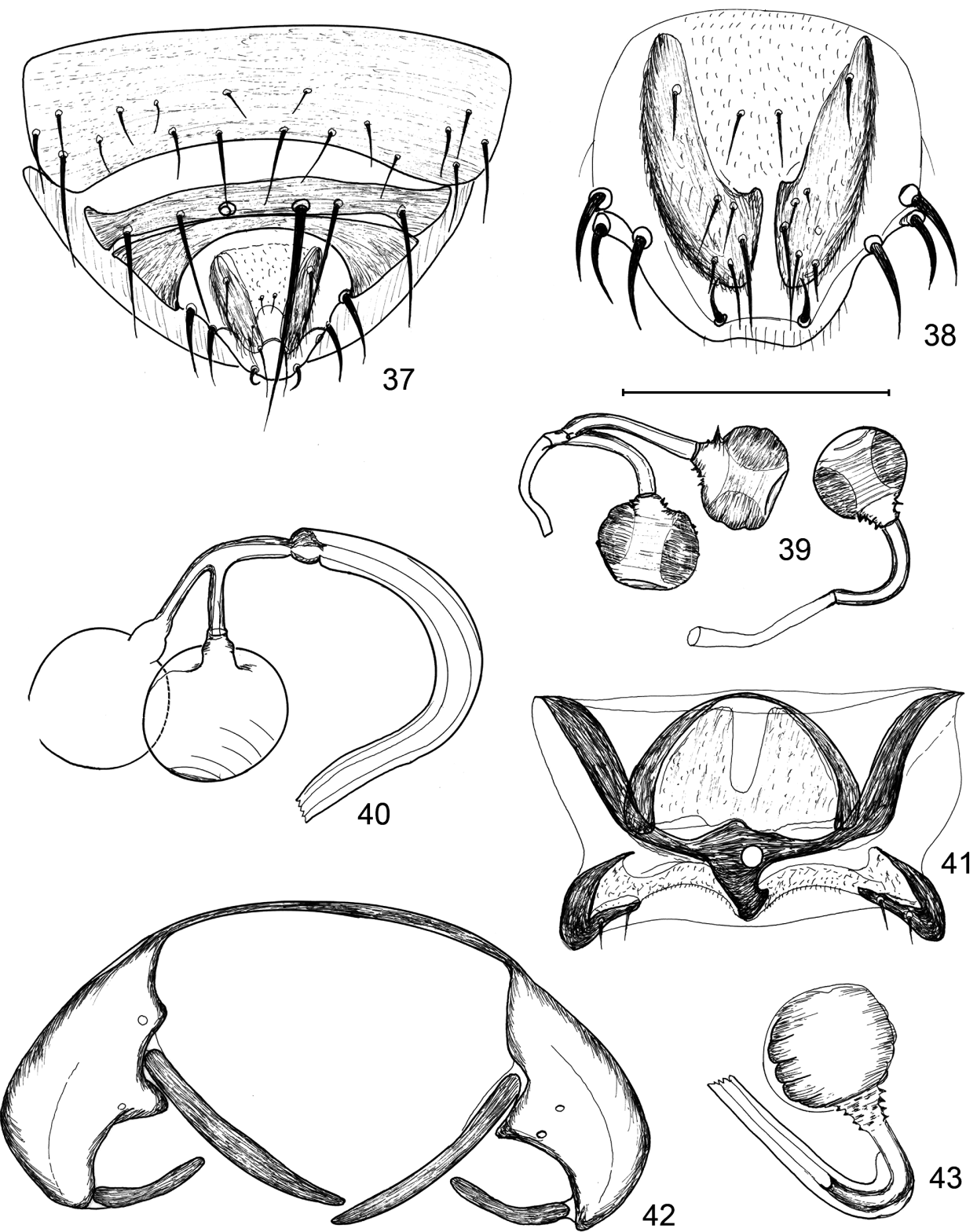

43

Figs 37-43. Pseudocollinella spp., female postabdomen and genitalia. 37-39= P. ochrea (L. Papp, 1974): 37 = postabdomen, dorsal view, 38 = epiproct, hypoproct and cerci, subdorsal-subcaudal view, $39=$ spermathecae; $40-43=P$. tunisica (L. Papp, 1974): $40=$ paired spermathecae, $41=$ sternite 8 in high magnification (not wholly symmetrical), $42=$ ventral structures of tergite 8 in high magnification, subcaudal view, 43 = unpaired spermatheca. Scale: $0.2 \mathrm{~mm}$ for Fig. 37, $0.1 \mathrm{~mm}$ for Figs 38-43. 
Pseudocollinella (Spinotarsella Richards, 1929), reinstated here as a subgenus.

Type species: Limosina humida Haliday, 1836 (mon.)

The most important features of the subgenus are listed below:

Inner orbital setae (2 pairs) always present.

A pair of very strong presutural acrostichal setae present.

Alula large and broad.

Contrarily to Pseudocollinella, male genitalia are with long hypandrial apodeme (rod).

Their subepandrial sclerite behind cerci (pseudocerci) is very broad $\mathrm{H}$ shaped, it consists of narrow plates; there are 2 narrow ventral plates (appendages), which emerge from the sagittal point of subepandrial sclerite.

The anterior surstylus is a transverse (+/- horizontal) triangle, whose lateral dorsal edge joins not much more than on one point to the edge of epandrium and hypandrium, as well as to the base of the posterior surstylus, its ventral posterior edge subapically joins to the process of the subepandrial sclerite. Posterior surstylus is simple, liguliform, its tip is not free, but connected there to the process of the subepandrial sclerite. Postgonite simple (Figs 48, 51-52). Distiphallus very short, basiphallus very short, forming a ring.

Other included species: Opacifrons jorlii Carles-Tolrá, 1990 (not studied now, see its original description).

\section{P. (Spinotarsella) humida (Haliday, 1836)}

Material studied: c. 530 specimens (HNHM) from Algeria, Tunisia, Crete, Greece, Georgia, Serbia, Bulgaria, England, Switzerland, Germany, Slovakia, Poland, Romania, Ukraine and mainly from Hungary; 49 specimens (Univ. Guelph Insect Collection): U.S.S.R., Siberia, S. A Marshall 1991: 30 : Novosibirsk Region, Cherny Mts, Lakeshore swamp, 22-29. vi.; 8 : Novosibirsk, Site 1, trail sweep, 27. vi.; 1 q : Novosibirsk, sweeps, poplar, mud, cattle, 22. vi.; 8 2 2 : Novosibirsk Region, Chany Lake Bio. Stn., on flowers, 3.

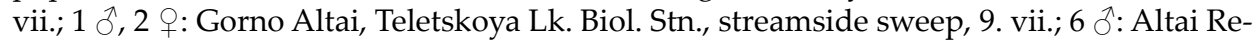
gion, Gorno Altai, roadside, seeps near river, 9. vii.; 2 o 39 : Altai region, Teletskoya Lake, 16-20. vii., pans in wet area, floaded, netties, sand; 1 : ibid., Teletskoya Lake, flooded pan traps in creek bed, 8-12.vii.; $1 \overbrace{}^{\Uparrow}$ : ibid., ca. 50 km Teletskoya Lk., 1500 m, pans, wet meadow, springs, open area, 11-12.vii.; 1 ' : ibid., $1500 \mathrm{~m}$. ca. $50 \mathrm{~km}$ SE of Teletskoya Lake, pans in wet moss, springs, open area, 11-12.vii. $1 \widehat{\jmath} 2$ 을 Altai Region, 1500 m, ca. 50 km SE Teletskoya Lake, sweep/malaise near cabin, 16.vii.; 1 : Altai Region, roadside - Typha, cow dung, nr. Barnaul, 21. vii.; 1 q: Altai Region, near Barawool, roadside typha, cow dung, 21. vi; 3 : Gorno Altai, Altai Station, streamside sweep, 9. vii.; 2 q: 100 km N Gorno Altai near Blysk, roadside sweeps, 9 . vi.

It is a Palaearctic species, the records from the Afrotropical region (VANsCHUYTBROECK 1950, 1951) are erroneous. 
Male sternite 5 without definite medio-caudal groove, caudal margin in some case with small hairy caudal lobe. The male genitalia of the specimens from England (type locality) and those from Siberia are not distinctly different. In order to demonstrate this, a figure each on (parameres) postgonites is given (Figs 51-52).

\section{P. (Spinotarsella) pseudohumida sp. $\mathrm{n}$.}

(Figs 44-50)

Holotype male (HNHM): P. R. CHINA: Fragrant Hill [Xiangshan] Park, West Mountains Nat. Reserves - 40 km NW Beijing, rocky creek shore, July 1, 1992, leg. L. Papp.

Paratypes (HNHM): 16 males 7 females: same as for the holotype.

Measurements in mm: body length 2.20 (holotype), 2.08-2.30 (paratypes), wing length 2.47 (holotype), 2.24-2.60 (paratypes), wing width 1.10 (holotype), 1.06-1.18 (paratypes).

In body characteristics this new species is very similar to those of $P$. (S.) humida. The specific differences are in the male genitalia only.

Male sternites 4 and 5 less broad than in $P$. (S.) humida, sternite 5 (Fig. 44) rather long with a small semicircular medio-caudal groove, which is filled up by a thinly sclerotised part with a pair of thin setae. Synsternite (Figs 45-46) with a rather long sternite 6 part, particularly so medially. Sternite 6 part narrowed on the right side and does not reach the right edge of sternite 8 part. Sternite 7 part continued into a medially directed narrow lobe. Cercal part of epandrium (Fig. 47) with 2 pairs of setae: a long and a medium-long ones. Subepandrial sclerite with a pair of very long ventral lobes, which bear short setae. Anterior surstylus (Fig. 49) small, short and broad (i.e. transverse and medially directed), apically rounded with 6 pairs of apical-subapical setae. Posterior surstylus (Fig. 50) of an intricate form, medial apex weakly sclerotised, rather long basally with a dark narrow caudal process. Postgonite (Fig. 48, cf. Figs 51-52) with longer anterior lobe basally, apical 2/3 straight evenly narrow with gently rounded apex.

Female of the new species cannot be separated from that of P. humida.

Etymology. The specific epithet of this new species 'pseudo' (Greek-Latin: false) + humida refers to the specific epithet of its close relative.

P. (Spinotarsella) pseudohumida sp. n. is a species close to the widespread $P$. (S.) humida. They are different in the details of male genitalia only. Male sternite 4 and 5 are very broad in $P$. (S.) humida, less broad in P. (S.) pseudohumida. Male synsternite different, sternite 7 part longer towards the right abdominal edge than in humida. Anterior surstylus longer than in humida, posterior surstylus distinctly different, less long basally. Postgonite of $P$. (S.) pseudohumida is with longer dorsally directed anterior lobe basally, apical 2/3 straight evenly narrow with gently rounded apex; anterior basal process subtriangular and anteriorly directed; apical $2 / 3$ of postgonite is slightly narrowed in humida. 


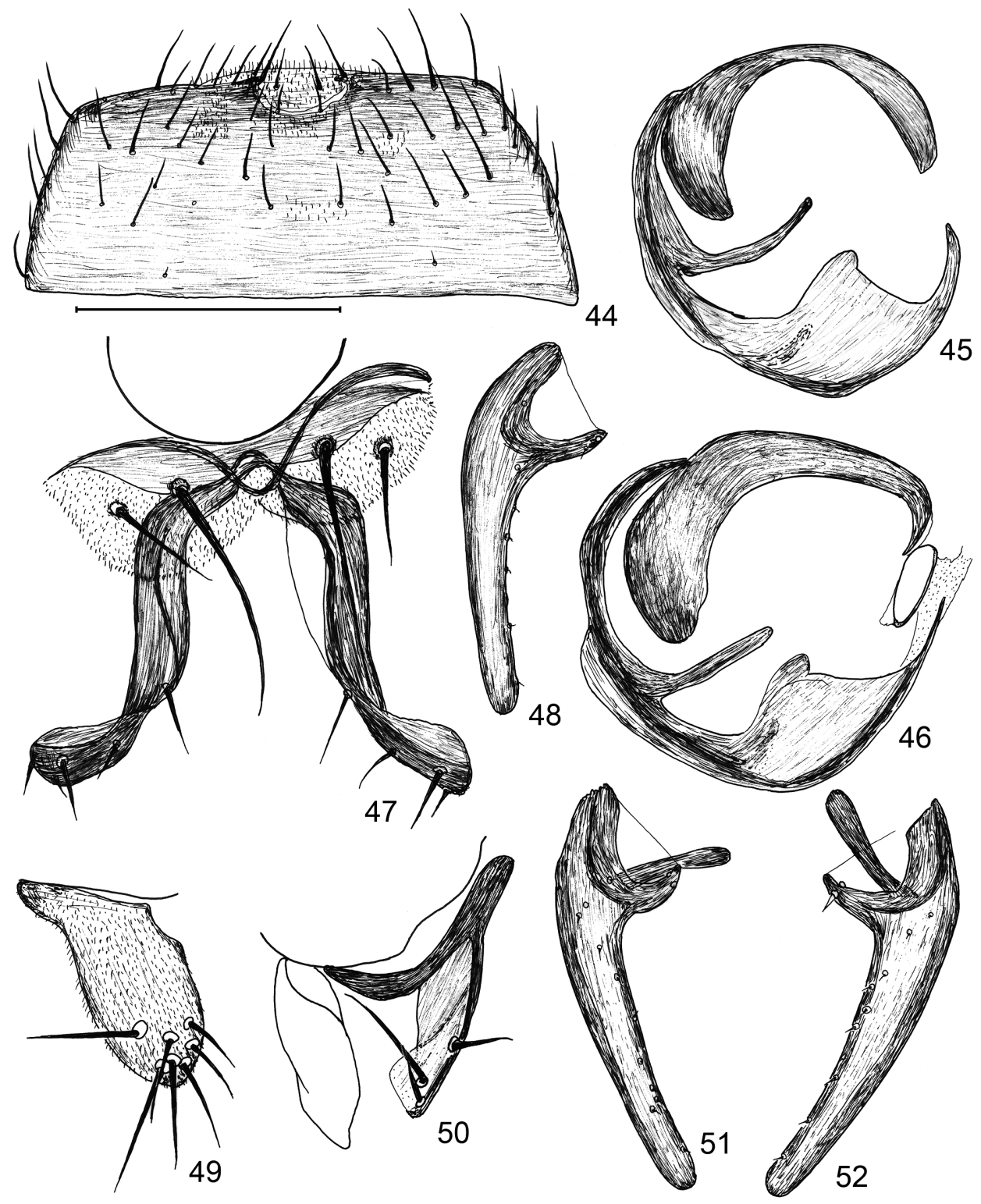

Figs 44-52. Pseudocollinella (Spinotarsella) spp., male postabdomen and genitalia. 44-50=P. (S.) pseudohumida sp. n.: $44=$ sternite 5 , ventral view, 45-46 = synsternite of two specimens in a slightly different subcaudal view, 47 = subepandrial sclerite with its appendix, caudal view, $48=$ postgonite, broadest (a slightly sublateral) view, $49=$ anterior surstylus, broadest (subanterior-sublateral) view, $50=$ posterior surstylus with the contours of the epandrium and the anterior pair, subcaudal-sublateral view; 51-52 = P. (S.) humida (Haliday, 1836), postgonite in broadest extension: $51=$ a specimen from England (type locality), $52=$ a specimen from Sibiria. Scale: $0.2 \mathrm{~mm}$ for Figs 44-46, $0.1 \mathrm{~mm}$ for Figs 47-52. 
In the collection of the HNHM there is a female of Spinotarsella from N Vietnam (Sín Chai), which belongs possibly to this new species, or to a third species. This is the most southern occurrence of the subgenus.

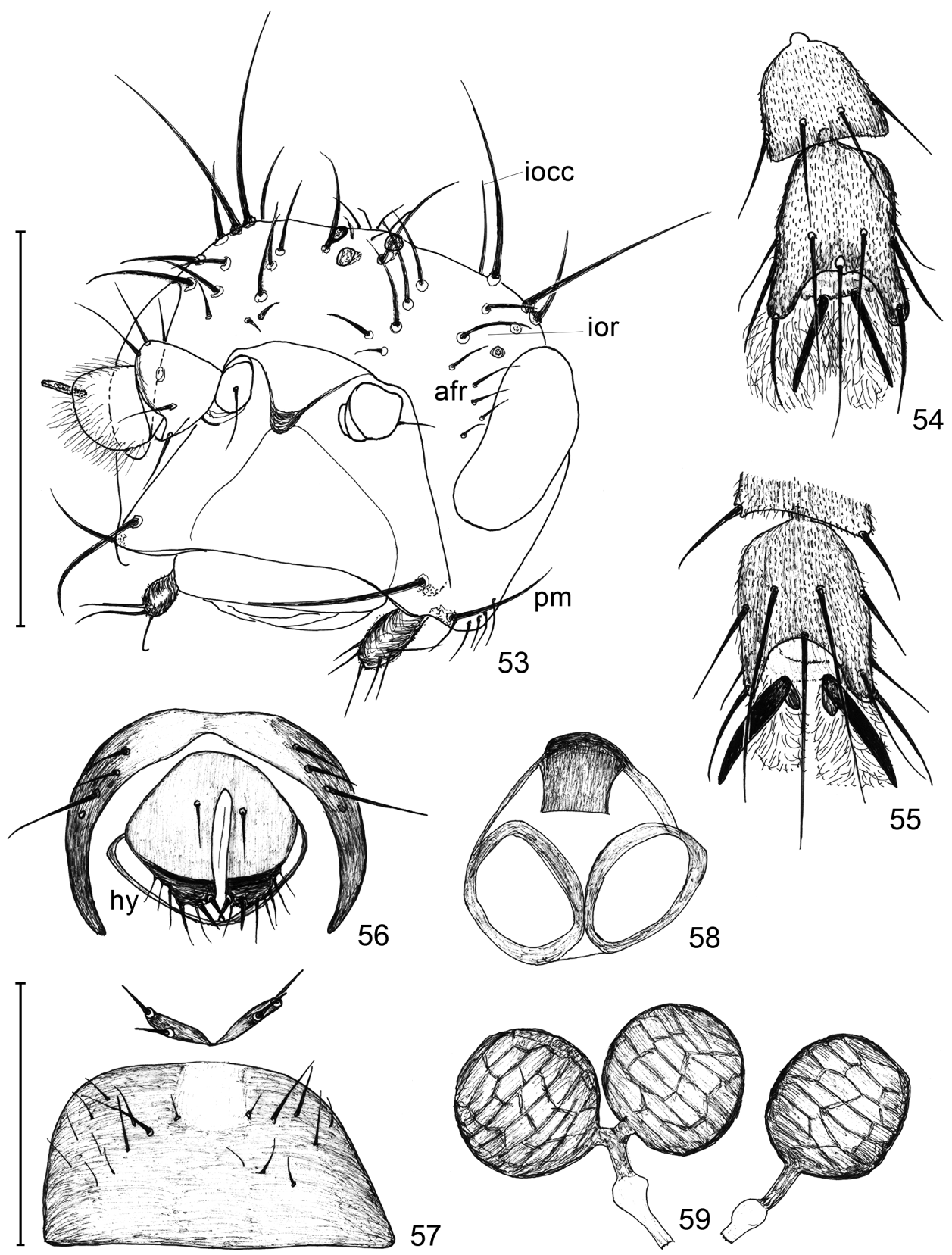




\section{Setiopacifrons subgen. nov.}

Type species: Leptocera (Opacifrons) dupliciseta Duda, 1925 (orig. des., designated here)

Gender: feminine.

Head (Fig. 53). 1 short anterior plus 3 strong posterior interfrontal pairs. Arista with very long, i.e. more than $0.12 \mathrm{~mm}$, cilia (in a majority of species). 1 strong katepisternal seta. Presutural (= anterior intra-alar) seta rather close to notopleural seta.

Last (5th) tarsomere of one or more legs may be modified both in males and female: fore last tarsomere (Fig. 54), or hind last tarsomere (Fig. 55).

Wing membrane brownish. Alula small and narrow, liguliform, i.e. not broad, apex rounded.

Male sternite 5: generally bipartite: a broad anterior part with a concave medio-caudal part, which embracing posterior smaller part; both with or without processes.

Sternite 6 part of the synsternite is usually with a sagittal modification (i.e. not a paired one).

Male cerci sometimes with a pair of thinly sclerotised ventral processes, otherwise cerci fused to epandrium, and so below we are speaking about "the cercal part of epandrium".

Hypandrium rather triangular, apodeme (medial rod) short or missing, arms strong.

Subepandrial sclerite without any modification. Subepandrial sclerite broad, joins to posterior surstyli (to their mid dorsal edge) by a pair of thin very short extensions.

Two pairs of surstyli, anterior pair is usually simple, posterior one usually with stronger setae and in almost every species with a blunt curved apical process.

Phallus short, phallapodeme long, epiphallus present.

Postgonites (parameres) bilobed in majority of the species, or actually in 2 parts: the "normal" posterior part plus an anterior, large, ventrally directed part, which is +/- separated from the posterior part. Between caudal edges of postgonites there is another lamella (very thin) which seems double more caudally and both turn dorsally.

Female sternite 7 various, sternite 8 (Fig. 57) very small in 2 parts, cerci strongly fused to epiproct (Fig. 56), hypoproct rather thin. Spectacles-shaped sclerite (Fig. 58) short but rather well-sclerotised; it is similar to that of the Opacifrons spp. (PAPp 2012: figs 49, 52) but medial part much shorter. Spermathecae (Fig. 59) globular with net-like surface.

Biology - Although we may expect numerous other undescribed species in the new subgenus in the future, their habitat seems to have been well-defined. All specimens with some more detailed label data are from "over/along a brooklet/creek" in the tropical-subtropical areas up to Korea and Japan. We do not know enough as for the closer circumstances. It is sure that they do

Figs 53-59. Pseudocollinella (Setiopacifrons subgen. n.), general features. $53=P$ (S.) dupliciseta (Duda, 1925), head (left fronto-orbital setae omitted); $54=P$. (S.) prima sp. n., male fore tarsomere 5 , dorsal view; $55=P$. (S.) vulnerata sp. n., female hind tarsomere 5 , dorsal view; 56-59 $=P$. (Setiopacifrons) sp. (?paradupliciseta sp. n.) female genitalia: $56=$ tergite 8 , epiproct, cerci and hypoproct, subdorsal view, $57=$ sternites 7 and 8 in situ (sides of sternite 7 much upcurved), ventral view, $58=$ spectacles-shaped sclerite, $59=$ spermathecae (afr: anterior fronto-orbital setulae, hy: hypoproct, ior: inner orbital setae, iocc: inner occipital seta, p: first peristomal seta). Scales: $0.2 \mathrm{~mm}$ for Figs 53, 56-57, $0.1 \mathrm{~mm}$ for $54-55,58-59$. 
not occur at all the small brooklets, e.g. we did not capture any of them in the course of our two collection trips to Vietnam in 2009 and 2011, contrarily to concentrated efforts.

Taxonomy - Description of 16 new species in a new subgenus is not a common finding even in such a large dipterous family as Sphaeroceridae. However, a closer analysis of the features of the male genitalia shows that body characteristics, some genital characteristics in comparison to other genital characteristics are not in harmony. The relationships in the subgenus are good examples for a mosaic, or network evolution. For instance, one of the most conspicuous features of a number of species, i.e. the modification of 5th tarsomeres seems to be a convergent feature, i.e. must not be used for finer phylogenetic analysis. I am afraid that many more species are to be described until the phase when a phylogenetic analysis will result in the establishment of satisfactory phyletic relations.

Etymology. The name of the new subgenus is a combination of the Latin 'seta' and the generic name Opacifrons, referring to its resemblance to that genus.

\section{SETIOPACIFRONS SPECIES FROM THE AFROTROPICAL REGION}

\section{P. (Setiopacifrons) congoana sp. $\mathrm{n}$.}

(Figs 60-70)

Holotype male (IRSNB): Malaise traps, lowland evergreen primary forest (disturbed) - D. R. Congo: Oriental Prov., Likombo forest, 2 km SW Bomona, 1.28349, 23.72319, 20-22. v. 2010, A. H. Kirk-Spriggs. - [yellow] Bayekoli Ebale Congo Expedition 2010 - BECE 00822.

Paratypes: 1 male (HNHM): same as for the holotype, BECE 00803. 1 male (BMSA): Malaise traps, lowland evergreen swamp forest - D. R. Congo: Oriental Prov., Eydo forest, ca. 2 km E Lieki, 0.69642, 24.186, 2. vi. 2010, A. H. Kirk-Spriggs. - [yellow] Bayekoli Ebale Congo Expedition 2010 - BECE 02623. 1 male (BMSA, gen. prep.): Burundi: Bururi Prov., Réserve Naturelle Forestière Kigwena $04^{\circ} 09.949^{\prime} S 2^{\circ}$ 30.455' E, 17-20. xi. 2010, 810 m, - [blue] Entomology Dept., National Museum, Bloemfontein, South Africa - BMSA (D) 24469.

Measurements in mm: body length 1.91 (holotype), 1.84-1.94 (paratypes), wing length 1.73 (holotype), $1.70-1.88$ (paratypes), wing width 0.78 (holotype), $0.75-0.81$ (paratypes).

Inner orbital setae comparatively short, and also 1-2 short setulae beside them. 3 pair of long ifr (longest $0.14 \mathrm{~mm}$ ), plus a short anterior pair.

Figs 60-66. Pseudocollinella (Setiopacifrons) congoana sp. n., male sternite 5 and genitalia. $60=$ sternite 5, ventral view, 61 = synsternite, subventral view, 62 = cercal part of epandrium, broadest (subcaudal) view, 63 = subepandrial sclerite (slightly flattened), anterior view, $64=$ postgonite in broadest (sublateral) view , 65 = phallus and phallapodeme, lateral view, $66=$ basiphallus and basal part of distiphallus in higher magnification, lateral view. Scale: $0.2 \mathrm{~mm}$ for Figs 60-61, 65, $0.1 \mathrm{~mm}$ for Figs 62-64, 66. 
None of the last tarsomeres modified. Subbasal seta on costal vein short, setae on first costal section nearly as long subbasal one. Costal section $20.57 \mathrm{~mm}$, 3rd section $0.54 \mathrm{~mm}$. Inter-crossvein section of M1+2 $0.225 \mathrm{~mm}, \mathrm{M}-\mathrm{M}$ crossvein $0.12 \mathrm{~mm}$.

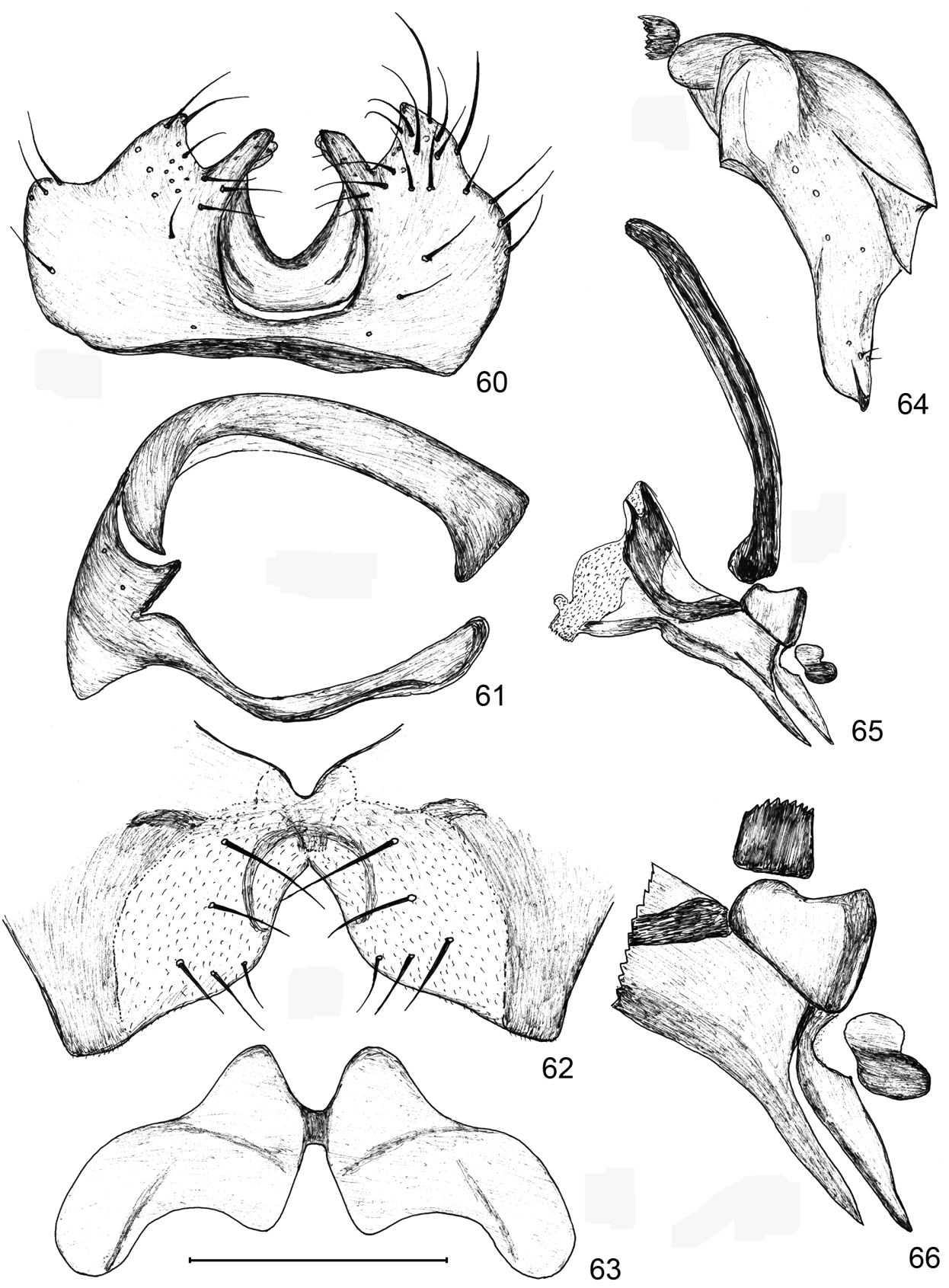


Male sternite 5 (Fig. 60) peculiar: larger anterior part rather long, with 2 pairs of caudal processes: one pair of triangular lateral and one pair of digitiform, medially curved processes. Medial (in other species, caudal) part of sternite 5 simple, without setae. Synsternite (Fig. 61) is also characteristic: sternite 6 part broad and thin, no medial sclerite or modification. Sternite 7 part rather small and not broad, sternite 8 part is not long but comparatively longer on the right side. Cercal part of epandrium (Fig. 62) forms two subtriangular extension with rather few and short setae. Subepandrial sclerite (Fig. 63) rather broad and comparatively short. Hypandrium (Fig. 67) without hypandrial apodeme but with an extremely broad medial part. Anterior surstylus (Fig. 70) with long base and medially even longer, and very characteristic as it is divided longitudinally: its medial part is much

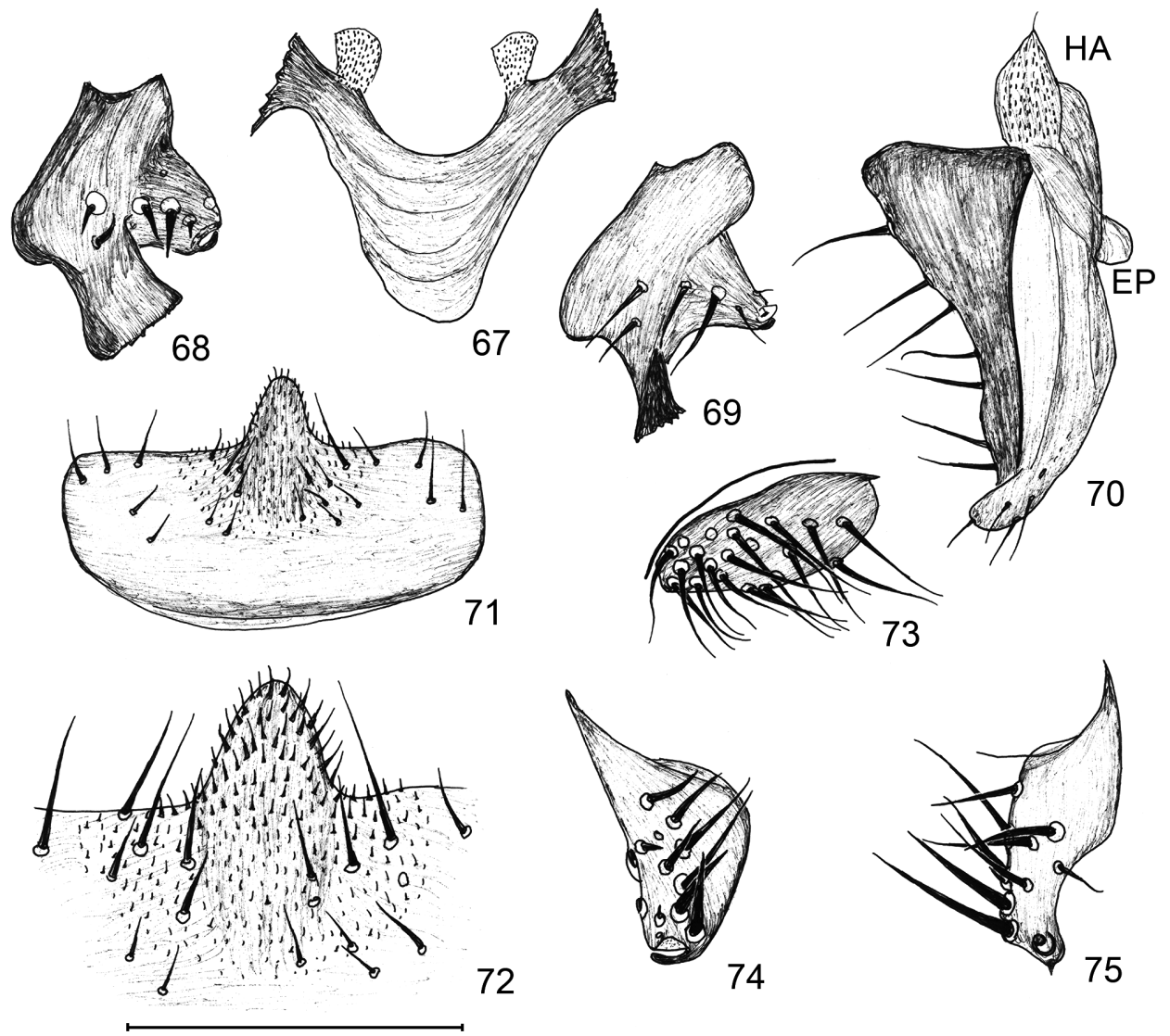

Figs 67-75. 67-70 = Pseudocollinella (Setiopacifrons) congoana sp. n., male genitalia. $67=$ hypandrium, ventral view, $68=$ posterior surstylus, ventral view, $69=$ posterior surstylus, sublateral view, $70=$ anterior surstylus with small parts of epandrium and hypandrium. $71-75=P$. (S.) normalis sp. $n$., male sternite 5 and genitalia: $71=$ sternite 5 , ventral view, $72=$ medio-caudal part of sternite 5 in higher magnification, 73 = anterior surstylus in broadest extension (subventral view), $74=$ posterior surstylus, broadest (submedial) view, $75=$ posterior surstylus, ventral view (EP: epandrium, HA: hypandrium). Scale: $0.2 \mathrm{~mm}$ for Figs 67, 71, $0.1 \mathrm{~mm}$ for Figs 68-70, 72-75. 
darker than basal part. Posterior surstylus (Figs 68-69) short and broad with mediumlong but thick setae, caudally with two apices. Postgonite (Fig. 64) not divided, apical part narrowed with a short true apex. Basiphallus (Fig. 66) small with a long thin and pointed epiphallus and with an extra sclerite caudally to epiphallus; distiphallus (Fig. 66) with a long thin and pointed appendix in lateral view, rather similar to epiphallus.

Female unknown.

Etymology. The specific epithet of the new species refers to its type locality (Congo).

\section{P. (Setiopacifrons) normalis sp. n.}

(Figs 71-75)

Holotype male (HNHM): Tanzania, Muyuni, Morogoro reg., 1-18. II. 1987, leg. Mahunka, Zicsi.

Paratypes (HNHM): 1 male (gen. prep.), 1 female: same as for the holotype.

Measurements in mm: body length 1.81 (holotype), 1.80-1.94 (paratypes), wing length 1.98 (holotype), 1.92-1.98 (paratypes), wing width 0.82 (holotype), $0.80-0.84$.

Anterior inner orbital setae $2 / 3$ as long as posterior one, with $1-2$ additional short setulae. None of the last tarsomeres modified. Subbasal seta on costal vein strong $(0.12 \mathrm{~mm})$, other setae on first costal section short $(0.04-0.05 \mathrm{~mm})$. Second costal section $0.64 \mathrm{~mm}$, 3rd section $0.62 \mathrm{~mm}$. Inter-crossvein section of M1+2 $0.25 \mathrm{~mm}, \mathrm{M}-\mathrm{M}$ crossvein $0.13 \mathrm{~mm}$.

Male sternite 5 (Figs 71-72) rather simple: sternite 5 rather long with a limited number of medium-long setae. Its medio-caudal part extended into a caudal broad and narrowly rounded process, which bears only short setae (Fig. 72). Hypandrium without medial apodeme (rod). Anterior surstylus (Fig. 73) rather long medially broadly rounded and covered almost evenly with long and rather thick setae. Posterior surstylus (Figs 74-75) is very characteristic: broadly rounded in broadest view with long thin apical process, otherwise with long and very thick setae, best seen in ventral view.

Female. Not separable from those of the related species; the female paratype was designated just based on the same locality label.

Etymology. The specific epithet of the new species (Latin: normal) refers to the simple (not modified) last tarsomeres ot this species.

\section{P. (Setiopacifrons) prima sp. $\mathrm{n}$.}

(Figs 54, 76-85)

Holotype male (HNHM): Ghana, Banda Nkwanta - 1965 VIII. 19., Endrődy-Y.[ounga], Nr. 58.

Paratypes: 1 female (HNHM): ibid., VIII. 1-5., Nr. 48.; 1 female (HNHM): ibid., VIII. 6-9., Nr. 49. 1 male 1 female: Ghana, Kumasi, 25.6. 1969. No. 376, S. Endrődy-Y.[ounga].

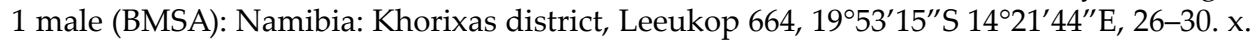
2001, A.H. Kirk-Spriggs \& E. Marais, Malaise trap sample riverbed - [blue] Namibian Natural Insect Collection, National Museum P.O. Box 1203, Windhoek, Namibia. 1 male: ibid.,

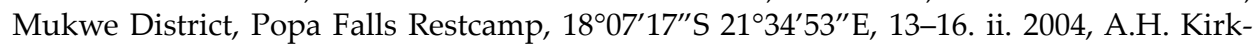




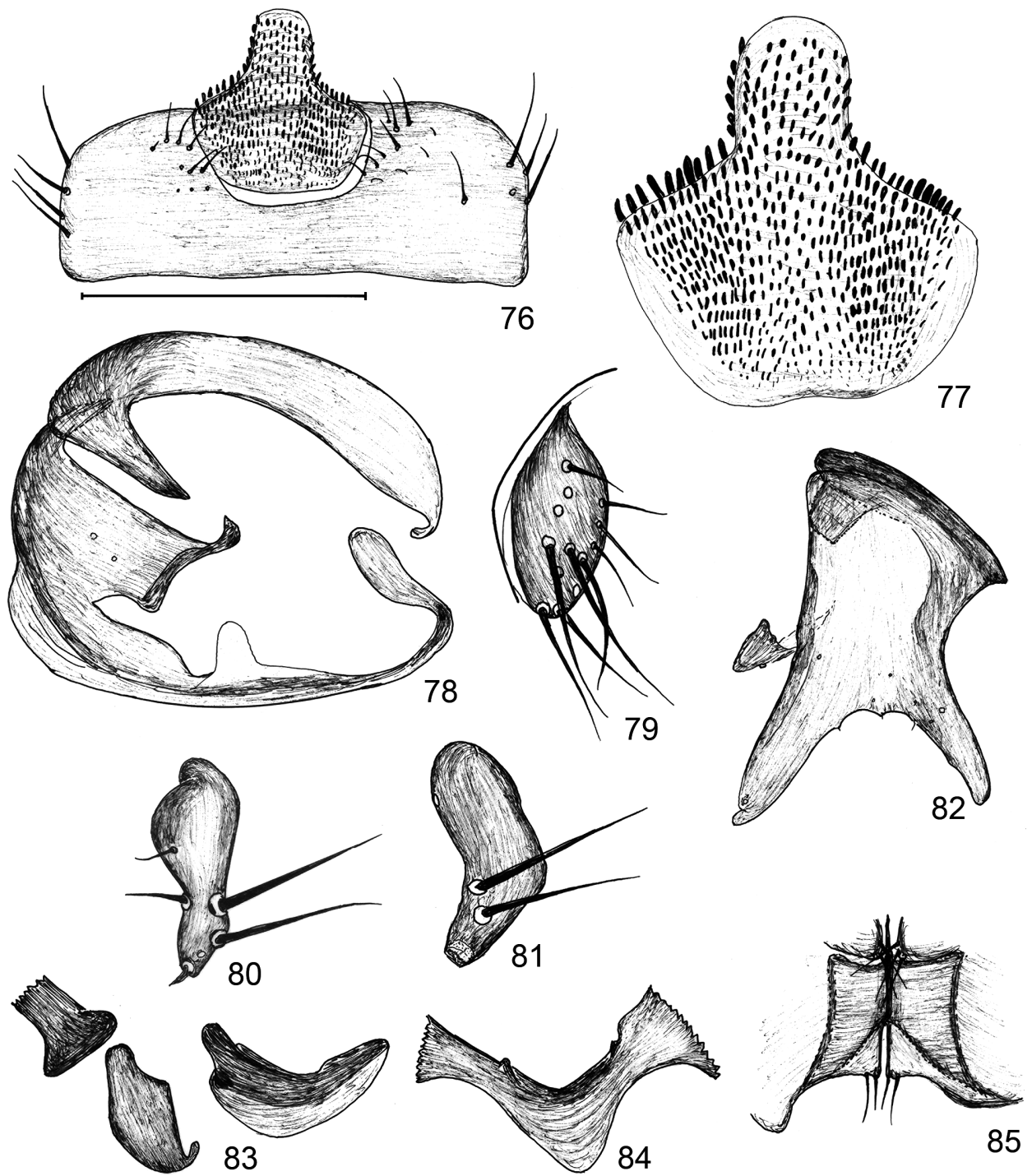

Figs 76-85. Pseudocollinella (Setiopacifrons) prima sp. n., male sternite 5 and genitalia. $76=$ sternite 5, ventral view, 77 = sternite 5, medio-caudal part in higher magnification, ventral view, $78=$ synsternite, subventral view, $79=$ anterior surstylus, broadest (subventral) view, $80=$ posterior surstylus, anterior-subventral view, cercal part of epandrium, broadest (subcaudal) view, 81 = posterior surstylus, broadest (subanterior) view, $82=$ postgonite in broadest (sublateral) view, 83 = basiphallus with base of phallapodeme, lateral view, $84=$ hypandrium, ventral view, 85 = cercal part of epandrium and subepandrial sclerite, caudal view. Scale: $0.2 \mathrm{~mm}$ for Figs 76, 78, 84-85, $0.1 \mathrm{~mm}$ for Figs 77, 79-83. 
Spriggs, Malaise trap, riverine forest; 1 male: Sweeping lowland evergreen swamp forest - D.R. CONGO, Oriental Prov., Eyolo forest, ca. 2 km E Lieki, 0.69642, 24.24186, 25-29. v. 2010, A. H. Kirk-Spriggs. - [yellow] Bayekoli Ebale Congo Expedition 2010 - BECE 02622. 1 female: ibid., Likombo forest, $2 \mathrm{~km}$ SW Bomane, 1.28349, 23.72358, 20-22. v. 2010 - Malaise traps, lowland evergreen primary forest (disturbed) - BECE 00539.

Measurements in mm: body length 1.98 (holotype), 1.87-2.12 (paratypes, wing length 1.97 (holotype), 1.94-2.08 (paratypes), wing width 0.88 (holotype), 0.85-0.90 (paratypes).

Two long inner orbital pairs. Gena behind vibrissa $0.07 \mathrm{~mm} .1+1$ anterior peristomal setae plus a strong ventrally placed genal seta. 3 strong palpal setae. Legs ochre, ventral row of thorns on male mid tibia weaker. Mid and hind last tarsomeres usually normal, fore last tarsomere (Fig. 54) modified with dorsal thick black claws, almost as long as tarsomere. Costal section $20.65 \mathrm{~mm}$, 3rd section $0.62 \mathrm{~mm}$. Inter-crossvein section of M1+2 $0.27 \mathrm{~mm}$, $\mathrm{M}-\mathrm{M}$ crossvein $0.13 \mathrm{~mm}$.

Male sternite 5 (Figs 76-77) strongly species-specific: anterior part with longer setae only laterally, medio-caudal part without setae but covered by flat black pegs (Fig. 77). There is a broad rounded sagittal process on the medial part. Synsternite (Fig. 78) with strongly extended sternite 6 part on the right side and with a small sagittal caudal extension; sternite 7 part lengthened medially, sternite 8 part rather short. Subepandrial sclerite (Fig. 85) small with angular edges dorsally, cercal part of the epandrium angular with 2 pairs of short setae. Hypandrium (Fig. 84) with out medial appendage but medial part broad and broadly rounded apically. Anterior surstylus (Fig. 79) comparatively small, rounded with some very long setae. Posterior surstylus (Figs 80-81) rather simple with 2 pairs of extremely long subapical setae; apical process rather short. Postgonite (Fig. 82) is unique: apical part divided into 2 narrow processes, its posterior part is not the posterior lobe of the Setiopacifrons species, but part of the anterior lobe. Basiphallus (Fig. 83) small with a small dorsally curved ventral process, epiphallus larger than basiphallus, more or less boat-shaped.

Female. Not separable from those of the related species; the female paratypes were designated just based on the same locality labels.

Etymology. The specific epithet of this new species refers to the modified fore last tarsomere (Latin 'prima' means first).

\section{P. (Setiopacifrons) setisternalis sp. n.}

(Figs 86-93)

Holotype male (BMSA, gen. prep.): Malaise traps, grassy floodplain - RSA: KZN, Ndumo Game R., pan at: $26^{\circ} 54.288^{\prime} \mathrm{S} 32^{\circ} 17.974^{\prime}$ E, 9-10. xii. 2009, A.H. Kirk-Spriggs [blue] Entomology Dept., National Museum P.O. BOX 266, Bloemfontein 9300, South Africa-BMSA (D) 19266.

Measurements in mm: body length 1.92 (holotype), wing length 1.76, wing width 0.79.

Three strong ifr pairs and 1 short anterior pair. Subbasal costal seta $0.15 \mathrm{~mm}$, other setae on first costal section $0.10 \mathrm{~mm}$. All last tarsomeres normal. 
Male sternite 5 (Fig. 86) is species-specific (although structurally similar to that of $P$. (S.) vulnerata): medio-caudally deeply hollowed as in a number of the related species but it has no medio-caudal extension. Instead, there is a V-shaped darker plate sagittally, sternite 5 bears extremely long thick setae latero-caudally and short but thick medially curved setae on sides of medial hollow. Synsternite (Fig. 87) form a closed ring, which is a very rare phenomenon in the species of the subfamily Limosininae. Sternite 6 part short with a small less sclerotised anterior process, sternite 8 part comparatively long. Subepandrial sclerite (Fig. 88) shortened but better sclerotised and melanised sagittally. Hypandrium simple,
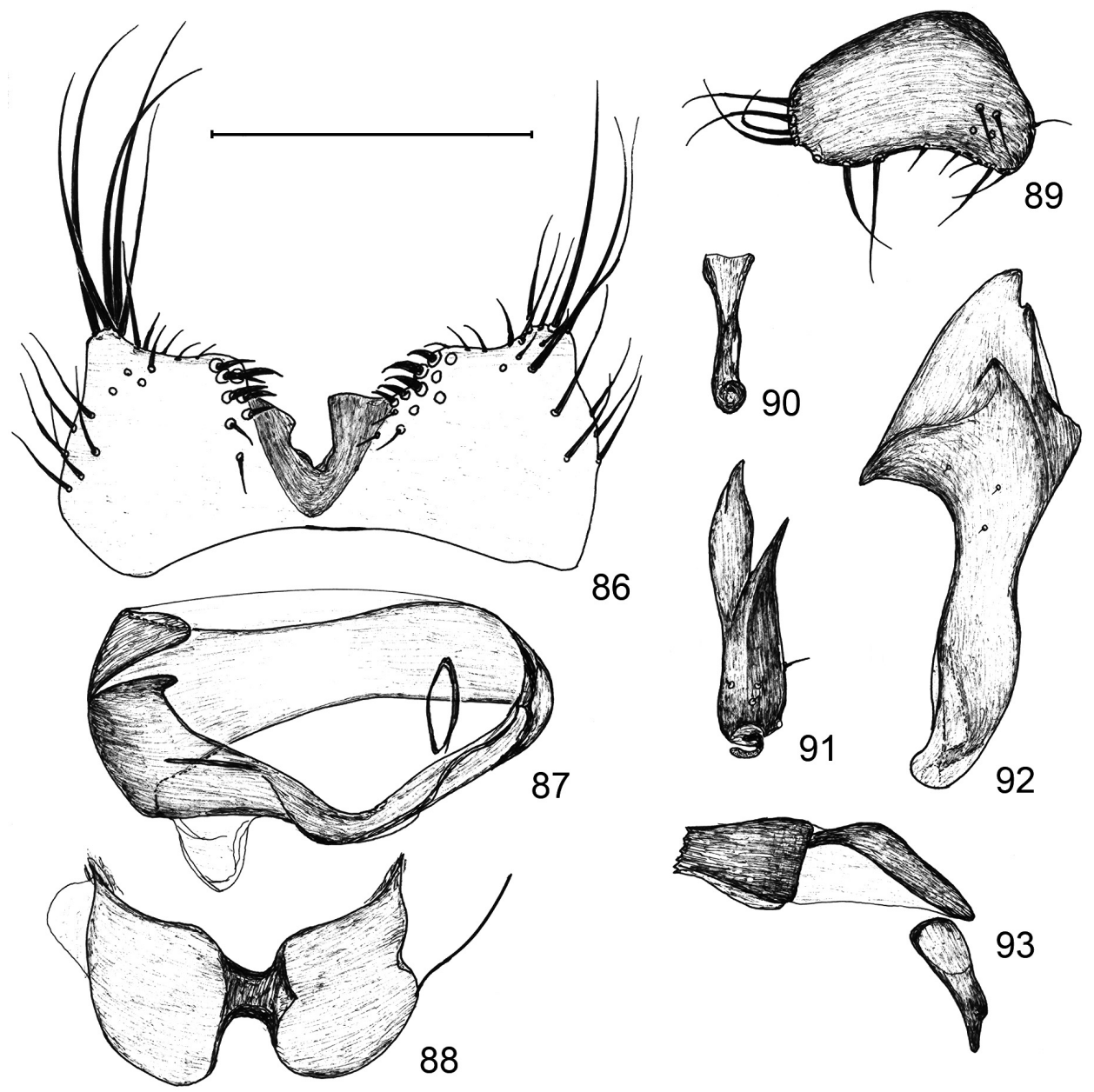

Figs 86-93. Pseudocollinella (Setiopacifrons) setisternalis sp. n., male sternite 5 and genitalia. $86=$ sternite 5 , ventral view, 87 = synsternite, ventral view, $88=$ subepandrial sclerite, anterior view, 89 = anterior surstylus, broadest (subventral) view, $90=$ ejaculatory apodeme, $91=$ posterior surstylus, anterior ventral view, 92 = postgonite, lateral view, $93=$ basiphallus and epiphallus with base of distiphallus. Scale: 0.2 mm for Figs 86-87, $0.1 \mathrm{~mm}$ for Figs 88-93. 
without medial appendage. Anterior surstylus (Fig. 89) comparatively broad, longer setae mostly on its margin. Posterior surstylus (Fig. 91) comparatively simple, long and narrow with thin curved apical process. Postgonite (Fig. 92) simple, i.e. anterior part not divided, posterior (basal) part short, not explicite. Ejaculatory apodeme (Fig. 90) bacilliform. Basiphallus (Fig. 93) long but not deep with a ventrally directed distinct epiphallus.

Etymology. The specific epithet of the new species refers to the extremely long setae on the male sternite 5 .

\section{P. (Setiopacifrons) vulnerata sp. n.}

$$
\text { (Figs 94-102) }
$$

Holotype male (IRSNB): Malaise traps, lowland evergreen swamp forest - D.R. CONGO: Oriental Prov., Eyolo forest, ca. 2 km E Lieki, 0.69642, 24.186, 25-29. v. 2010, A. H. Kirk-Spriggs. - [yellow] Bayekoli Ebale Congo Expedition 2010 - BECE 02350.

Paratypes: 3 males 4 females (BMSA): same as for the holotype $(02369,02330,03418$, 02371, 02352, 0.2353, 02363). 1 male 2 females (BMSA): ibid., 2.vi. (02626, 02620, 02621). 1 male 1 female (BMSA): ibid., Bosega, nr. Djabir village, 00.51814, 24.16574, BECE 03744, 03747, sweeping leaves primary lowland swamp forest. 1 male (BMSA): Burundi: Bururi Prov., Réserve Naturelle Forestière Kigwena $04^{\circ} 09.949^{\prime} S 2^{\circ} 30.455^{\prime}$ E, 17-20. xi. 2010, 810 m, - [blue] Entomology Dept., National Museum, Bloemfontein, South Africa. 2 males 1 female (HNHM): Tanzania, Kwasambia, Tanga region -1-18. II. 1987., leg. Mahunka, Zicsi.

Measurements in mm: body length 1.78 (holotype), 1.70-1.92 (paratypes), wing length 1.73 (holotype), 1.67-1.92 (paratypes), wing width 0.77 (holotype), 0.75-0.79 (paratypes).

Anterior part of frons reddish. Both inner orbital setae are weak, anterior pair c. 2/3 in length of the posterior pair. 6 rows of acrostichal microchaetae. Male mid tibia with a strong antero-ventral row of thorns, larger specimens also with a short apical postero-ventral row. All last tarsomeres modified (Fig. 55), fore claws are definitely shorter than tarsomere. Subbasal costal seta comparatively short, $0.12 \mathrm{~mm}$ only, other setae on first costal section $0.09-0.10 \mathrm{~mm}$. Second costal section $0.56 \mathrm{~mm}$, 3rd section $0.54 \mathrm{~mm}$. Inter-crossvein section of M1+2 0.225 , M-M crossvein $0.10 \mathrm{~mm}$.

Male sternite 5 (Fig. 94) strongly asymmetrical: medial part deeply hollowed without medial extension but with an asymmetrical bare dark plate, which seems to be fused to the rest of the sternite. Sternite 5 bears long setae latero-caudally but they are shorter than in $P$. (S.) setisternalis sp. n. Synsternite (Fig. 95) structurally same as in P. setisternalis, since it forms a ring (sides of sternite 6 and sternite 8 parts are almost completely fused), sternite 6 part short, sternite 8 part rather long, sternite 6 part with a broader, less sclerotised anterior lobe. Subepandrial sclerite (Fig. 98) is different from that of $P$. (S.) setisternalis, but sagittal part is shortened and better sclerotised, as in its related species. Anterior surstylus (Fig. 100) short and broad with short setae only. Posterior surstylus (Fig. 101) subdivided medially with rounded apex and with a long thin apical process. Ejaculatory apodeme (Fig. 99) definitely shorter than that of $P$. (S.) setisternalis sp. n. Postgonite (Fig. 97) with very small basal part and characteristic anterior part: a longer digitiform process which bears 2 apices and with a caudal shorter but pointed process. Phallus (Fig. 102) is unique with normal basiphallus but epiphallus is doubled: the body of the epiphallus bears an extra dorsal process, which joins to the body of epiphallus dorsally through a thin sclerite. 
Etymology. The specific epithet of the new species 'vulnerata' (Latin: wounded) refers to the modified last tarsomeres.

Four males and seven females from the D. R. Congo (Likombo, Eyolo, Bosega) must be related but not designated as paratypes: not all the 3 last tarsomeres modified but male genitalia seems to be identical or very similar to the male types of $P$. (S.) vulnerata.
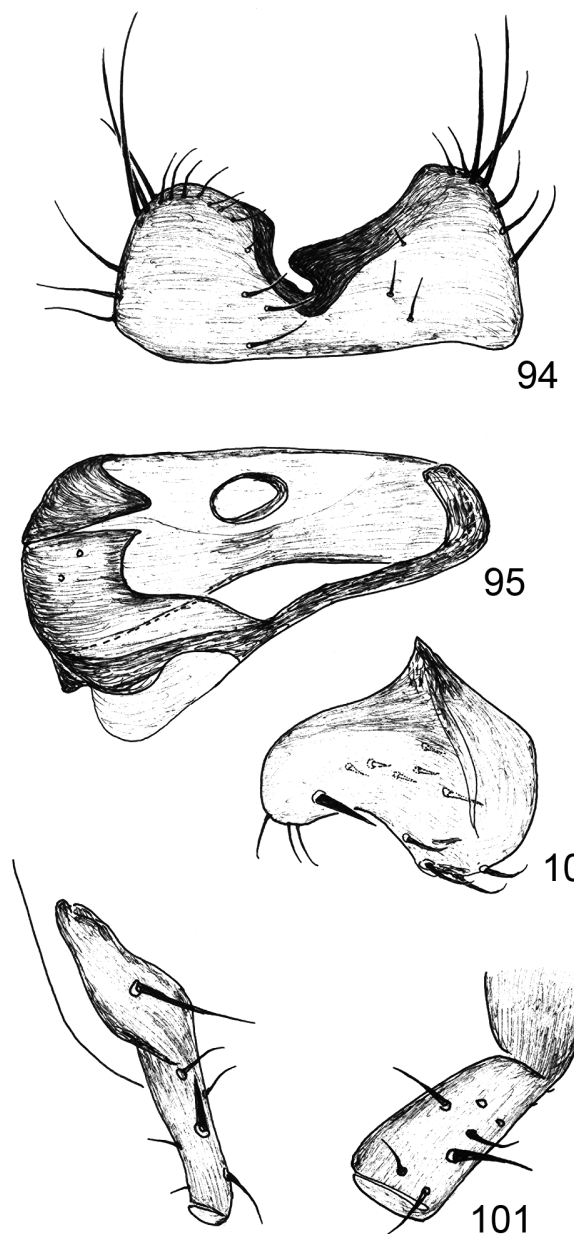

\section{4}
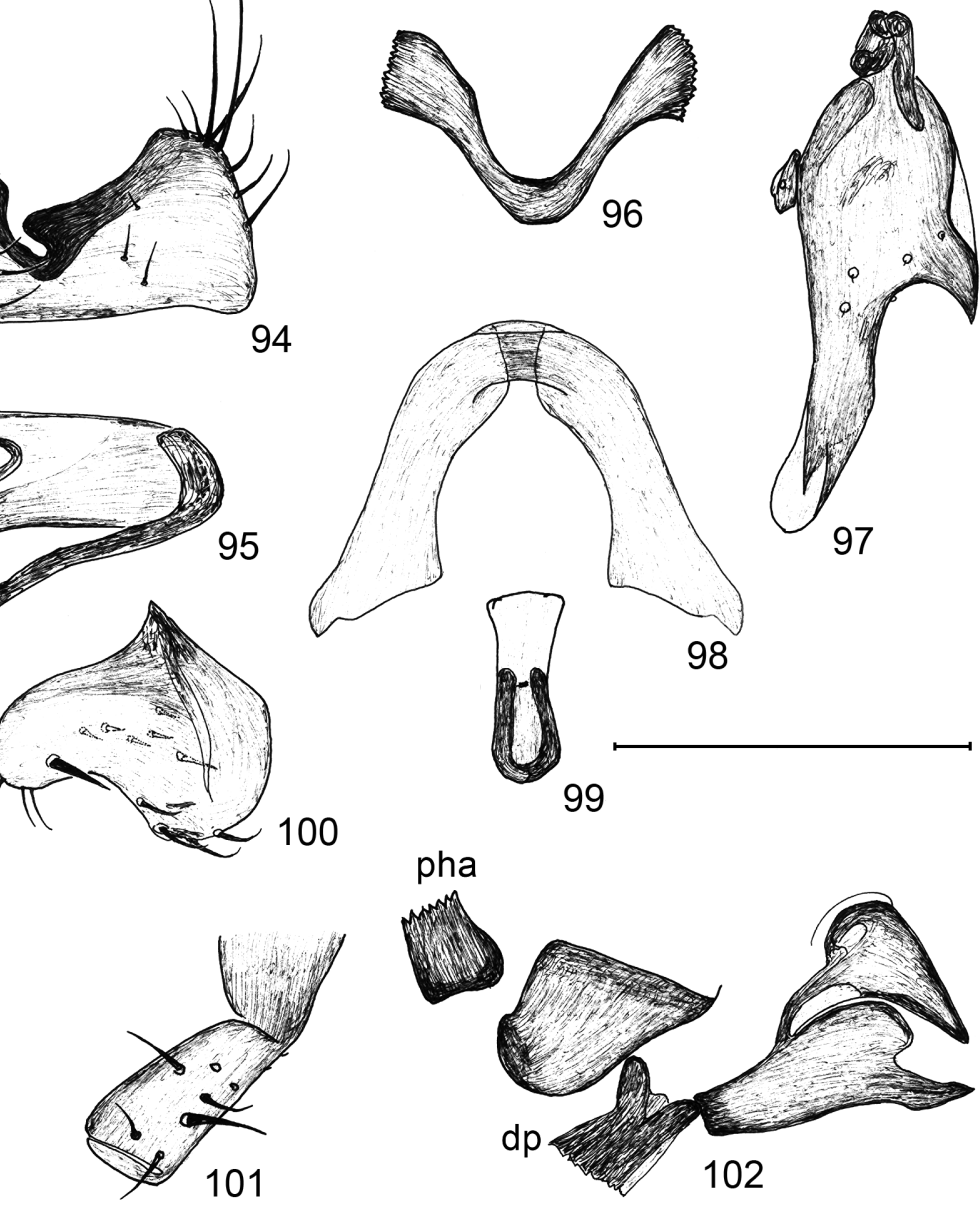

99

Figs 94-102. Pseudocollinella (Setiopacifrons) vulnerata sp. n., male sternite 5 and genitalia. 94 = sternite 5, ventral view, 95 = synsternite, subventral view, 96 = hypandrium, ventral view, $97=$ postgonite in broadest (lateral) view, $98=$ subepandrial sclerite, anterior view, 99 = ejaculatory apodeme, 100 = anterior surstylus, broadest (sublateral) view, 101 $=$ posterior surstylus in ventral view and in its broadest (sublateral) view, 102 = basiphallus, epiphallus with bases of distiphallus and phallapodeme (dp: distiphalus, pha: phallapodeme). Scale: $0.2 \mathrm{~mm}$ for Figs 94-96, $0.1 \mathrm{~mm}$ for Figs 97-102. 
Key to the Afrotropical species

of the subgenus Setiopacifrons (males)

(all species have short and apically rounded hypandrium without apodeme)

1. Male synsternite fused into a ring (Figs 87, 95). Medio-caudal part of male sternite 5 concave with an asymmetrical dark bare plate (Figs 86, 94). Postgonite not divided with short base (e.g. Fig. 92). 2

- Male synsternite not fused into a ring.

2. Last tarsomeres not modified. Male sternite 5 with extermely long setae on postero-lateral corner (Fig. 86). Medial part with a broad V-shaped bare plate. Anterior (large) part of the sternite with short thick medially directed setae on the medial margins. $\quad$ P. (S.) setisternalis sp. n.

- $\quad$ Last tarsomeres modified (Fig. 55). Postero-lateral corner of male sternite 5 (Fig. 94) more rounded with shorter setae. Medial part with strongly asymmetrical, extractor shaped bare plate (Fig. 94), medial margins of the anterior (large) part without medially directed setae.

\section{P. (S.) vulnerata sp. n.}

3. Medio-caudal part of male sternite 5 (Fig. 60) concave but its large anterior part rather long, with 2 pairs of caudal processes: one pair of triangular lateral and one pair of digitiform, medially curved processes. Anterior surstylus (Fig. 70) large, long based and medially even longer.

P. (S.) congoana sp. $n$.

- Medio-caudal part of male sternite 5 convex with a caudal sagittal lobe (Figs 71-72, 76-77). Anterior surstylus small, round (Figs 73, 79).

4. Last tarsomeres not modified. Caudal sagittal lobe of sternite 5 subtriangular with short hairs (Figs 71-72). Posterior surstylus only with c. 7 thick strong setae (Figs 74-75).

P. (S.) normalis sp. n.

- $\quad$ Last tarsomeres modified (Fig. 54), i.e. claws on fore tarsomere 5 are not V-shaped or curved but rod-like and placed on the dorsal edge of tarsus on one of more legs. Caudal sagittal lobe of sternite 5 (Figs 76-77) broad and broadly rounded with black flat pegs. Posterior surstylus (Figs 8082 ) with 2 thick strong setae only.

P. (S.) prima sp. n. 


\title{
SETIOPACIFRONS SPECIES \\ FROM THE ORIENTAL AND OTHER REGIONS
}

\author{
P. (Setiopacifrons) cederholmi (L. Papp, 1991), comb. $\mathbf{n}$. \\ (Figs 103-110)
}

Material studied: 1 male paratype (HNHM): [Sri Lanka] Centr. Prov., Kandy, 12. I. [1962], Loc. 9, swept on scrubs in jungle. This specimen is in a poor state of preservation as given by Papp 1991: 242) but its genitalia (in a plastic microvial) are still suitable.

All the Papp's figures (PAPP 1991: figs 46-49) in the original description are correct but the peculiarities of the genital parts were not interpreted correctly and so the distinctness of this species and of $P$. (S.) dupliciseta (Duda) were not realised that time.

Male sternite 5 (Fig. 103) in 2 distinct part, which are not connected sclerotically: anterior part with a pair of dark bare processes laterally to the medial (caudal) part; this medial (caudal) part continued into a sagittal caudal narrowly rounded hairy lobe; caudal edge of the medial part with black pegs, body of the medial part covered by minute dark thick hairs in groups of (2)-3-(4). Sternite 6 part of the synsternite with a larger triangular lobe perpendicularly directed into the genital vault. Anterior surstylus (Figs 109-110) very broad with numerous long setae. Posterior surstylus (Figs 104-106) rather broad but this is discernible only in sublateral-subventral view; posterior surstylus with and extra small lobe and with 2 pairs of very long thick setae; apical part narrow in ventral view, apical process comparatively small. Postgonite (Figs 107-108) is not properly shown in a given view: anterior lobe (Fig. 107) narrowed apically with a narrowly rounded apex; posterior part of postgonite's broadest view (Fig. 108) is a sublateral-subdorsal one, it is without peculiarities.

This species has been known only from its type specimens from Sri Lanka (Ceylon).

\section{P. (Setiopacifrons) communis sp. n.}

(Figs 111-122)

Holotype male (HNHM): Thailand: Mae Fang N.P., over \& along a forest brook Nov 1, 2004, No. 14, L. Papp \& M. Földvári.

Paratypes (all but 1 in HNHM): Thailand, L. Papp \& M. Földvári 2004: 22 males (1 in BMSA): same as for the holotype; 5 males: Nan Prov., Ban Na Lae nr Pua, 05. 11., over a rocky forest brook, No. 19; 1 male: Trang Prov., Ban Liphang, 16. 11., No. 34, over a shadowed, slow brook; 4 males: 8 km E Doi Anh Kang, over a rocky brook, Nov 2, No. 17. 

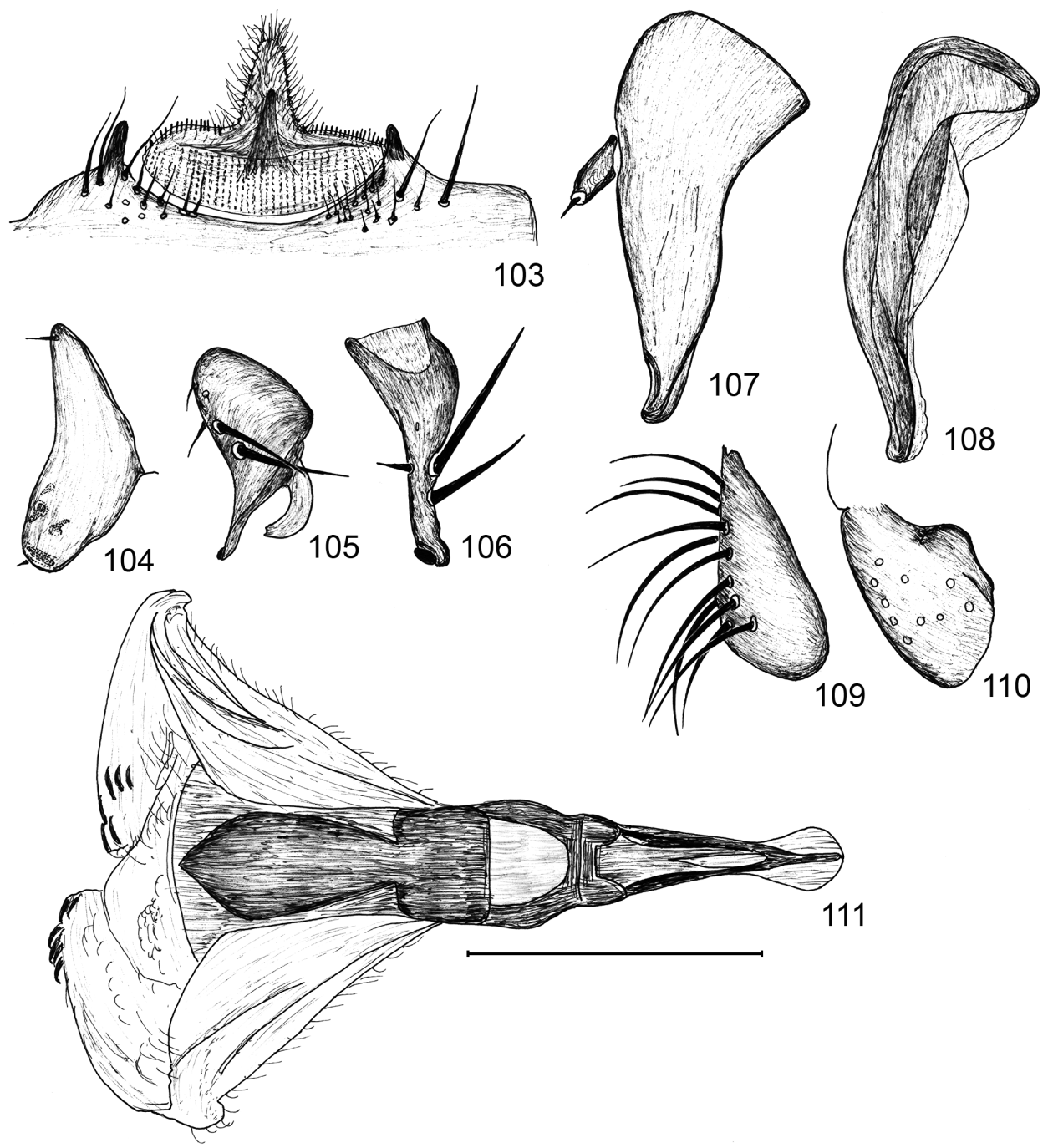

\section{3}


Measurements in mm: body length 1.60 (holotype), 1.45-1.92 (paratypes), wing length 1.65 (holotype), 1.48-1.76 (paratypes), wing width 0.71 (holotype), $0.69-0.76$ (paratypes).

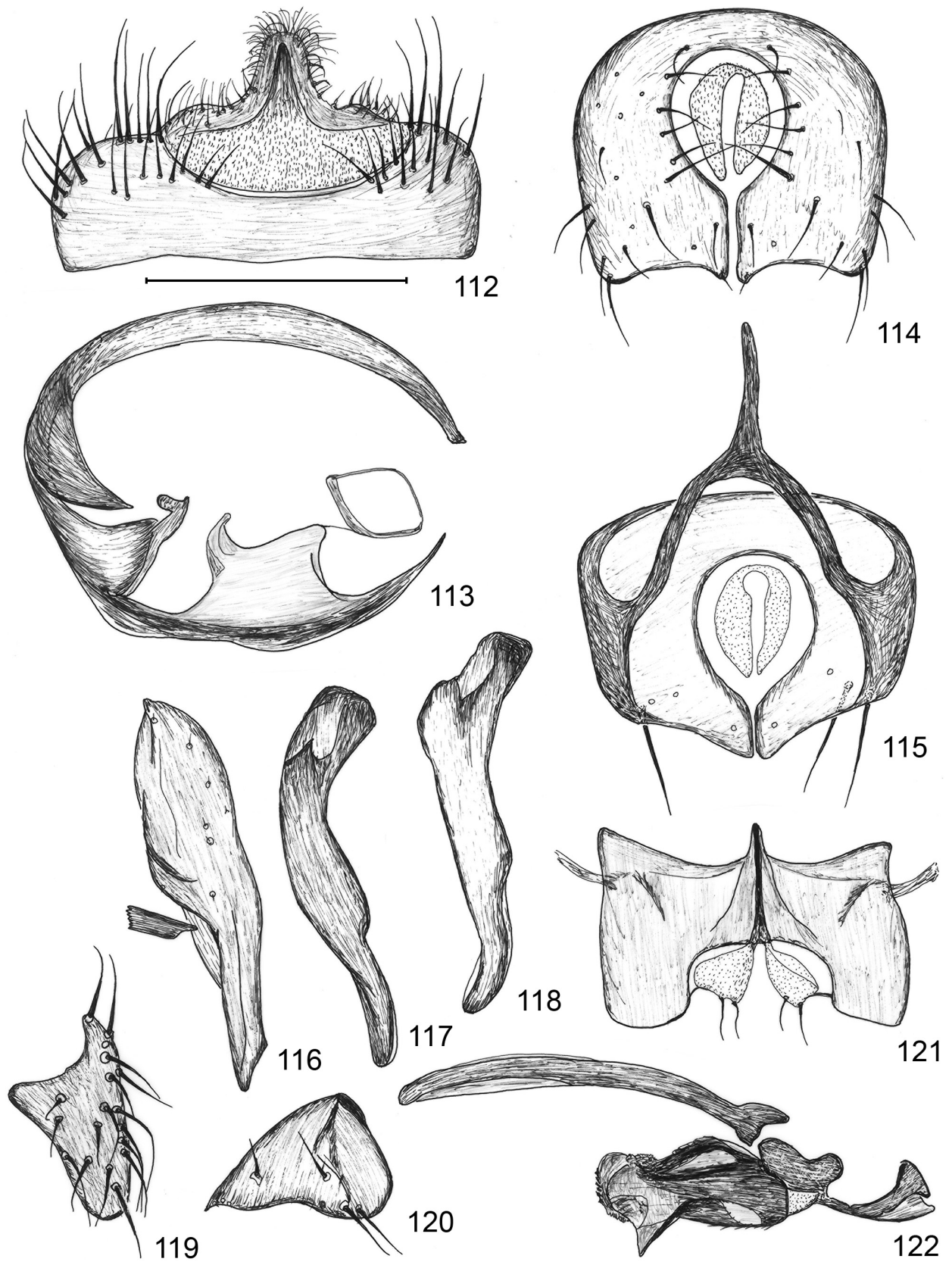


Three strong ifr pairs plus a short anterior pair. 2 comparatively strong inner orbital pairs, anterior pair 4/7 to 2/3 as long as posterior one. Most anterior part of frons reddish (reddish yellow). Gena narrower $(0.08 \mathrm{~mm})$ and yellowish. 2 strong palpal seta. Male mid tibia with ventral row of thorns particularly strong. None of the tarsomeres modified.

Male with medium long setae on epandrium. Male sternite 5 (Fig. 112) in 2 distinct part: anterior part with long setae laterally and caudally and with a deep and broad hollow to embrace medial (caudal) part. Caudal part of sternite 5 extended into a sagittal caudal, broadly rounded process, which bears hairs caudally (i.e. no black pegs on caudal margin as in cederholmi); body of the medial part without any setae. Synsternite (Fig. 113) is characteristic with a large medial caudal lobe on sternite 6 part, which is nearly perpendicular to the synsternite, and forms a part of the genital vault (! its concave part). Sternite 8 part of synsternite is short. Ventral (incl. cercal) part of epandrium (Fig. 114) not fused sagittally and without numerous setae. Hypandrium (Fig. 115) Y shaped: long thin with long hypandrial apodeme. Subepandrial sclerite (Fig. 121) shows the characteristic of the speciesgroup, membranously connected to the less sclerotised cercal process, the later bears 1 thin seta. Anterior surstylus (Fig. 118) with numerous but not very long setae. Posterior lobe (Fig. 120) subtriangular in its broadest view, apical process not separated. Postgonite (Figs 116-118) broadly bipartite: anterior lobe narrow with a narrow apical half and with a subtriangular apex; posterior part (lobe) of postgonite is given in two slightly different sublateral views (Figs 117-118). Apical (mostly membranous) part of distiphallus (Fig. 111) seems to be slightly asymmetrical but it is probably a consequence of its soft state. Phallic complex (Fig. 122) is similar to that of P. (S.) dupliciseta and P. cederholmi: basiphallus is small with a long, caudally directed epiphallus.

Etymology. The specific epithet of this new species 'communis' (Latin, meaning common) refers to the fact that this species was found in the highest number in the course of this study.

\section{P. (Setiopacifrons) dupliciseta (Duda, 1925), comb. n.} (Figs 123-132)

Material studied (all in the HNHM): Holotype male: Formosa Sauter - Taihorin, 1911. VII. - “Opacifrons dupliciseta $\widehat{\jmath}$ n. sp." Det. Dr. O. Duda. A female identified by O. Duda: Formosa Sauter - Takao, 1907. XII. 13. - “O. dupliciseta ㅇ" Det. Dr. O. Duda; Duda did not sign this female as a type specimen.

Newly collected material: 4 males 3 females: Taiwan: Pingtung Hsien, Kenting NP, Oct 5, 2000 - along a small brooklet, No. 18., leg. L. Papp; 1 female: ibid., Heng-Chun Trop.

Figs 112-122. Pseudocollinella (Setiopacifrons) communis sp. n., male sternite 5 and genitalia. $112=$ sternite 5 , ventral view, $113=$ synsternite, subventral-subcaudal view, $114=$ epandrium, caudal view, 115 = epandrium and hypandrium, ventral view (epandrial setae omitted), 116 = anterior part (lobe) of postgonite, sublateral-subcaudal view, 117-118= posterior part (lobe) of postgonite, in two slightly different sublateral views, $119=$ anterior surstylus, broadest (subventral) view, $120=$ posterior surstylus, broadest extension (subanterior view), 121 = cercal part of epandrium and subepandrial sclerite, caudal view, 122 = phallus and phallapodeme, lateral view. Scale: $0.2 \mathrm{~mm}$ for Figs 112-115, 122, $0.1 \mathrm{~mm}$ for Figs 116-121. 
Garden, light traps, Oct. 4-6, 2000, L. Peregovits \& L. Papp, No. 15; 1 male: Ilan Hsien, FuShan LTER Site, light traps, Sep. 26-27, 2000, leg. L. Ronkay, L. Peregovits \& L. Papp, No. 9.

Fortunately the male genitalia of the holotype is satisfactorily discernible without preparation of the genitalia.

The male and female which were reported from Vietnam by PAPP (1991) are misidentified; they have become the types of $P$. (S.) vietnamensis sp. $\mathrm{n}$.

Male sternite 5 (Fig. 124) almost completely separated into 2 parts: anterior part broad but short $0.11 \mathrm{~mm}$ long laterally (longest), medially deeply concave, embracing its medial (caudal) part, the two parts connected very narrowly on the left side distally. Medial part lengthened into a sagittal blunt process (Fig. 124), which is bicolorous, since medial part more sclerotised and melanised; distal part on all its margins with hair-like setae (i.e. without black pegs); anteriorly covered by very fine setulae ordered into lines; anterior part with long setae mostly on its caudal margin. Synsternite (Fig. 125) with its sternite 6 part in its medial 1/3 with a perpendicular very large lamella reaching more than $1 / 3$ depth of genital pouch i.e. concave part of the genital vault). Sternite 6 and 7 parts broadly fused laterally, free part of sternite 7 rather short, reaching only $3 / 8$ of the width of genital pouch. Sternite 8 part slightly longer than sternite 5 . Sternite 7 and 8 parts are not fused. Hypandrium with very thin rod (apodeme) (as in Fig. 115). Ventral (incl. cercal) part of the epandrium not fused sagittally, ventral half of the epandrium with numerous strong setae. Subepandrial sclerite (Fig. 126) different from that of P. (S.) communis but structurally they are very similar. Anterior surstylus (Fig. 127) broad and broadly rounded apically. Posterior surstylus (Fig. 128) subtriangular is its broadest view, apical process not separated. Postgonite broadly bipartite: anterior lobe (Fig. 129) almost evenly medium-broad with a small blunt apex, posterior lobe (Fig. 130) similar to that of $P$. (S). communis. Apical part of distiphallus (Fig. 131) more divided than that of communis. Phallic complex (Fig. 132) with small and less melanised basiphallus, and long caudally directed epiphallus.

Figs 123-132. Pseudocollinella (Setiopacifrons) dupliciseta (Duda, 1925), male sternite 5 and genitalia. 123 = right half of epandrium, caudal view, 124 = sternite 5, ventral view, $125=$ synsternite, subcaudal view, 126 = cercal part of epandrium and subepandrial sclerite, caudal view, $127=$ anterior surstylus, broadest (subventral) view, $128=$ posterior surstylus, broadest extension (subanterior-subcaudal view), $129=$ anterior part (lobe) of postgonite, sublateral-subcaudal view, $130=$ posterior part (lobe) of postgonite, sublateral view, $131=$ phallus, ventral view, 132 = phallus and phallapodeme, lateral view. Scale: $0.2 \mathrm{~mm}$ for Figs 123-125, 131-132, $0.1 \mathrm{~mm}$ for Figs 126-130. 


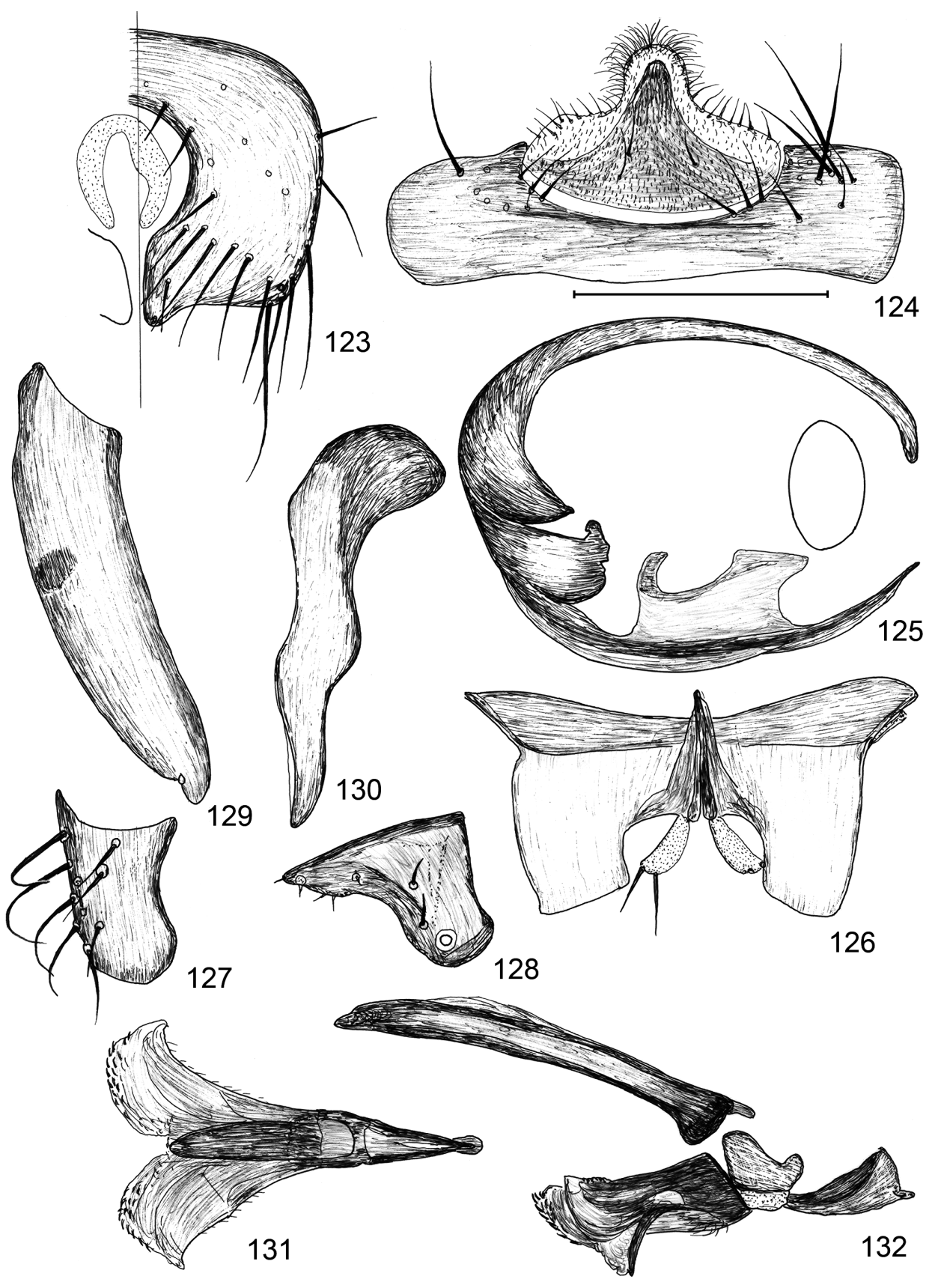




\section{P. (Setiopacifrons) formosensis sp. n.}

(Figs 133-142)

Holotype male (HNHM): Taiwan: Kaohsiung Hsien, Liukuei, Shan Ping LTER Site creek valley, No. 13, Mar 31-April 1, 2003, leg. L. Papp \& M. Földvári.

Paratype male (HNHM, gen. prep.): ibid., over/along a creek, April 2-3, 2003, leg. L. Papp, No. 15.

Measurements in mm: body length 2.06 (holotype), not measurable (paratype), wing length 1.74 (holotype), 1.70 (paratype), wing width 0.88 (holotype), 0.85 (paratypes).

Anterior inner orbital only $1 / 2$ of the posterior one. Subbasal seta on first costal section $0.13 \mathrm{~mm}$, some of the setae of first section are lengthened up to $0.10 \mathrm{~mm}$. Second costal section $0.69 \mathrm{~mm}$, 3rd section $0.61 \mathrm{~mm}$. Inter-crossvein section of M1+2 $0.26 \mathrm{~mm}, \mathrm{M}-\mathrm{M}$ crossvein $0.11 \mathrm{~mm}$. Only the right hind last tarsomere of the holotype is modified.

Male sternite 5 (Fig. 133) is spectacular, in 2 parts: medial (caudal part) much smaller than in P. dupliciseta. Anterior part with a semicircular convex "hole", lateral margins lengthened caudally with some small black pegs. Medial borders of the anterior part with medially directed long setae. Medial part with a sagittal dark process, whose apex is membranous and hairy; body of the medial part is covered by small black pegs (modified hairs). Synsternite (Figs 134-135): sternite 6 part with a peculiar quadrate process sagittally (this is seen as 2 dark ribs in caudal view, Fig. 135); sternite 7 part with a medially directed thin process; sternite 8 part with narrow and short ventral part on the left side. Cercal part of the epandrium with short dense thick setae. Subepandrial sclerite (Fig. 136) slightly reduced, comparatively short with more sclerotised and melanised sagittal part. Hypandrial apodeme very short. Anterior surstylus (Fig. 137) rather long with numerous long setae on its ventral part. Posterior surstylus (Figs 139-140) rather broad with 1 long setae and 2 peg-like processes: one subapical and one apical. Postgonite (Figs 141-142) peculiar with a broad anterior lobe, its apical third curved with a sharp apex; posterior (dorsal) part simple (Fig. 141). Basiphallus (Fig. 138) rather large with a large ventral apically rounded and caudally directed. Distiphallus narrower with 2 lateral dark laths.

Etymology. The specific epithet of this new species refers to Ilha Formosa, the original Portuguese name of the Taiwan island (its type locality).

\section{P. (Setiopacifrons) japonica sp. n.}

(Figs 143-152)

Holotype male (HNHM, gen. prep.): Japan, Kyushu Is., Oike, No. 6, 29.09.2006, 865 m, Mt. Kurodake area - N 33 07' 29.8' E $131^{\circ} 17^{\prime} 34.0^{\prime \prime}$, along stream, leg. M. Földvári.

Measurements in mm: body length 2.11 (holotype), wing length 2.05, wing width 0.84 .

Two strong palpal seta, anterior pm short $(0.07 \mathrm{~mm})$, aristal cilia $0.03 \mathrm{~mm}$. Also anterior interfrontal seta strong. Anterior interocellar seta definitely shorter than posterior one, 2 additional setulae there. Gena dark and broad $(0.08 \mathrm{~mm})$. Prescutellar acrostichal seta comparatively strong, $0.07 \mathrm{~mm}$ long. Legs brown. Fore coxa definitely more than $1.0 \mathrm{~mm}$. 
Mid tibia with strong ventral row of thorns. All last tarsomeres and claws normal. Costal setae as in koreana. Second costal section $0.64 \mathrm{~mm}$, 3rd section $0.64 \mathrm{~mm}$. Inter-crossvein section of M1+2 $0.26 \mathrm{~mm}, \mathrm{M}-\mathrm{M}$ crossvein $0.13 \mathrm{~mm}$.

Male sternite 5 (Figs 143-144) bipartite and similar to that of $P$. (S.) dupliciseta. Larger anterior part (Fig. 144) with numerous long setae and a pair of black blunt processes caudally on the edge of its central groove. Posterior part (Fig. 143) without long setae but with a large caudally directed, not pointed sagittal lobe; caudal margin of posterior part with longer flat black pegs, less caudally with similar small pegs; sagittal lobe bears fine setulae.

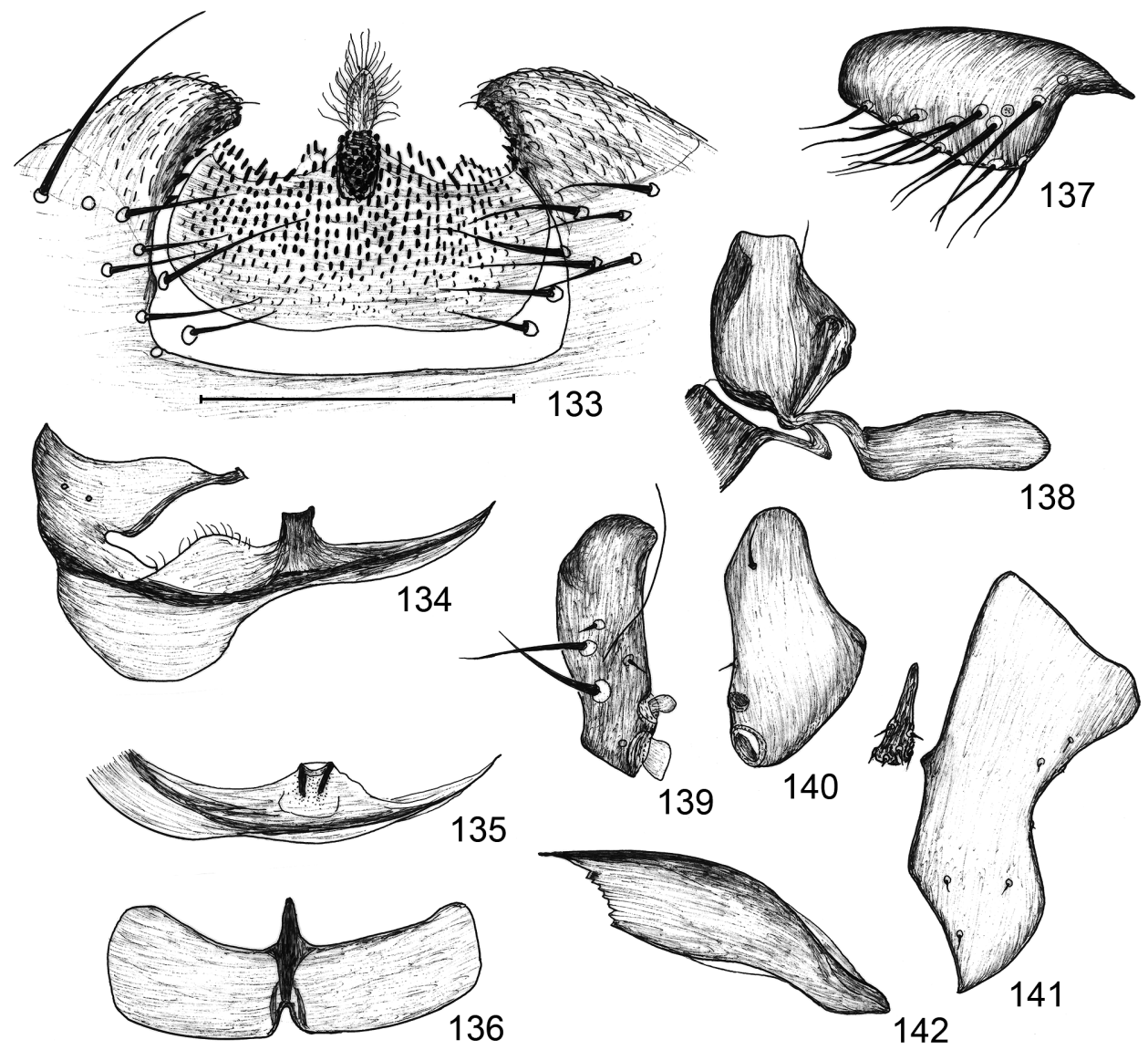

Figs 133-142. Pseudocollinella (Setiopacifrons) formosensis sp. n., male sternite 5 and genitalia. $133=$ medio-caudal part of sternite 5 in high magnification, $134=$ sternite 6 and 7 parts of synsternite, ventral view, $135=$ sternite 6 part of synsternite, caudal view, $136=$ subepandrial sclerite, anterior view, 137 = anterior surstylus, broadest (subventral) view, $138=$ basiphallus and epiphallus with base of distiphallus, 139 = posterior surstylus, subcaudal view, $140=$ posterior surstylus, broadest (sublateral) view, $141=$ anterior part of postgonite, broadest (sublateral) view, $142=$ posterior part of postgonite, broadest (sublateralsubdorsal) view. Scale: 0.2 mm for Figs 134-135, $0.1 \mathrm{~mm}$ for Figs 133, 136-142. 


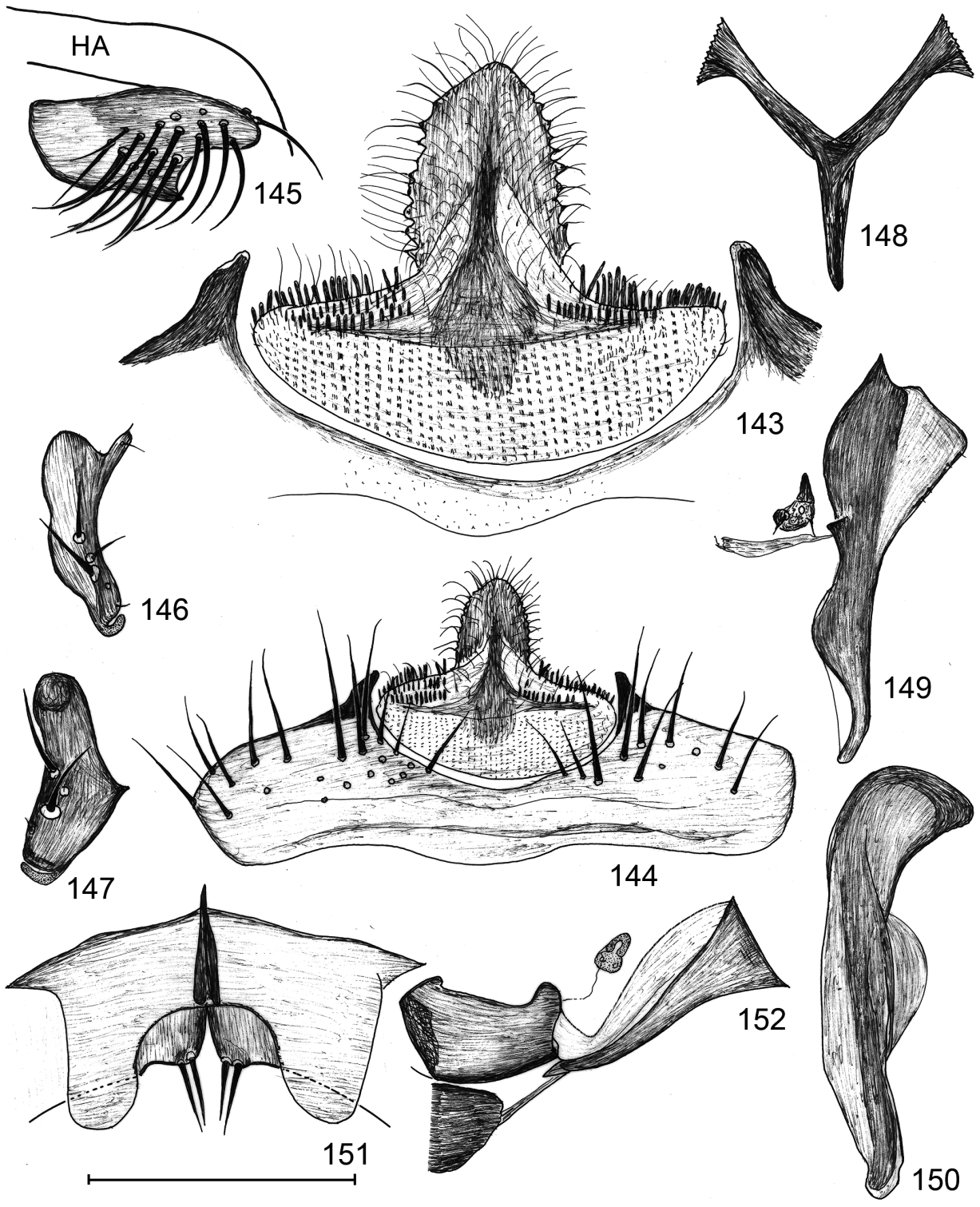

Figs 143-152. Pseudocollinella (Setiopacifrons) japonica sp. n., male sternite 5 and genitalia. $143=$ medio-caudal part of sternite 5 in high magnification, $144=$ sternite 5 , ventral view, 145 = anterior surstylus, broadest (subventral-submedial) view, $146=$ posterior surstylus, anterior ventral view, 147 = posterior surstylus, broadest (anterior subventral) view, $148=$ hypandrium, ventral view, 149= anterior part of postgonite, broadest (sublateral) view, 150 = posterior part of postgonite, broadest (lateral-subcaudal) view, 151 = cercal part of epandrium and subepandrial sclerite, anterior view, $152=$ basiphallus and epiphallus with base of distiphallus, lateral view (HA: hypandrium). Scale: $0.2 \mathrm{~mm}$ for Figs 144, 148, 0.1 mm for Figs 143, 145-147, 149-152. 
Cercal part of epandrium (Fig. 151) with 2 pairs of thick ventral setae. Subepandrial sclerite (Fig. 151) broad and overruns ventral edge of epandrium. Hypandrium (Fig. 148) with distinct but thin apodeme (rod) and with thin arms. Anterior surstylus (Fig. 145) in medial

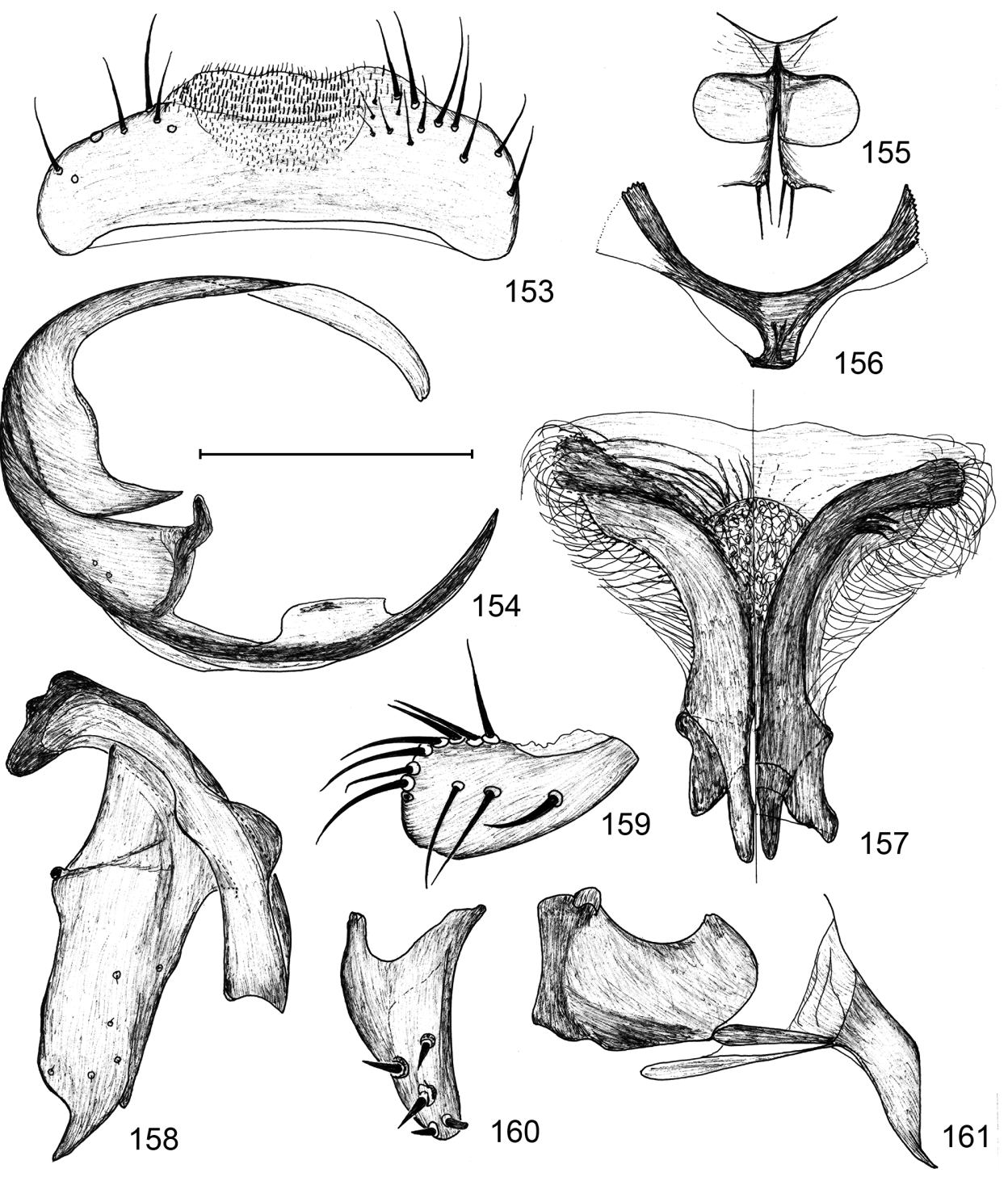

Figs 153-161. Pseudocollinella (Setiopacifrons) koreana sp. n., male sternite 5 and genitalia. $153=$ sternite 5 , ventral view, $154=$ synsternite, subanterior view, $155=$ cercal part of epandrium and subepandrial sclerite, anterior view, 156 = hypandrium, ventral view, $157=$ phallus, ventral view, $158=$ postgonite, broadest (sublateral) view, $159=$ anterior surstylus, broadest (subventral) view, $160=$ posterior surstylus, broadest (subanterior-subventral) view, 161 = basiphallus and epiphallus, lateral view. Scale: $0.2 \mathrm{~mm}$ for Figs 153-154, 156, 
margin with a distinct edge, covered with long setae, except for its apical part. Posterior surstylus (Figs 146-147) broadest in lateral-subcaudal view, with 2 long setae and strongly curved comparatively thin apical process. Postgonite (Figs 149-150) broadly bifid, anterior lobe rather narrow with thin but not sharp apical part, posterior lobe comparatively broad and positioned rather dorsally. Basiphallus (Fig. 152) normal with a caudal extension and with large long, caudally directed epiphallus. Ejaculatory apodeme short.

Etymology. The specific epithet of the new species refers to its type locality (Japan).

\section{P. (Setiopacifrons) koreana sp. n. (Figs 153-161)}

Holotype male (HNHM, gen. prep.): Korea, Prov. Ryang-gang, Plateau Chann-pay, San-zi-yan, 1600 m, 27. Aug. 1971. - No. 205, leg. S. H. Horvatovich et J. Papp.

Measurements in mm: body length 1.95 (holotype), wing length 2.14, wing width 0.84 .

Two pairs of strong inner orbital setae but no additional setulae beside them. 3 pair of strong ifr, plus a short anterior seta. None of the tarsomeres modified. Subbasal setal pair of costa $0.15 \mathrm{~mm}$, other seta on first section $0.10 \mathrm{~mm}$. Second costal section $0.69 \mathrm{~mm}$, 3rd section $0.64 \mathrm{~mm}$. Inter-crossvein section of M1+2 $0.31 \mathrm{~mm}, \mathrm{M}-\mathrm{M}$ crossvein $0.12 \mathrm{~mm}$.

Male sternite 5 (Fig. 153) rather simple: anterior part comparatively long with long setae sublaterally and caudally. Posterior (medial) part without a sagittal process or long setae, and covered by short thick hairs. Synsternite (Fig. 154) with its sternite 6 part in its medial $1 / 3$ with an almost perpendicular short but broad lobe, better seen in subanterior view. Cercal part of epandrium (Fig. 155) fused on a shorter sagittal line, edge with 2 pairs of thin setae. Subepandrial sclerite small and rounded. Hypandrium (Fig. 156) without apodeme, anterior part broad and rounded apically. Anterior surstylus (Fig. 159) rounded apically, its medium-long setae are mainly on its medial edge. Posterior surstylus (Fig. 160) with 5 short thick and pointed setae. Postgonite (Fig. 158) with a robust anterior lobe which is broad except its apical 1/10; there much narrowed with a proclinate sharp edge; posterior lobe characteristic in shape, oblique, over the anterior lobe. Phallus (Figs 157, 161) with large blunt basiphallus and long epiphallus, epiphallic apex is very narrow and slightly dorsally curved. Distiphallus (Fig. 156) with very long and dense hairs laterally, and with a long thin connecting sclerite to epiphallus (Fig. 161).

Female unknown.

Etymology. The specific epithet of the new species refers to its type locality (N Korea).

\section{Pseudocollinella (Setiopacifrons) nasalis (Richards, 1973)}

(Figs 162-167)

Material studied (HNHM): 1 male (gen. prep.): Australia, Qld, Boonah Bunjurgen, farm, 25. 08. 2005, No. 5 - 28 $02^{\prime} 52^{\prime \prime S} 152^{\circ} 37^{\prime} 61^{\prime \prime E}$, waterhole, leg Földvári \& Kun. 1 male (gen. prep.) Australia, NSW, Round Hill, No 80.15 km N from camping. 11. Jan. 1981, Hangay. 
Male sternite 5 (Fig. 162) is of an intricated form: anterior part simple, its central groove not deep, with numerous medium-long setae. Posterior (central) part with a blunt caudal process sagittally and all surface (except for a small anterior medial part) covered

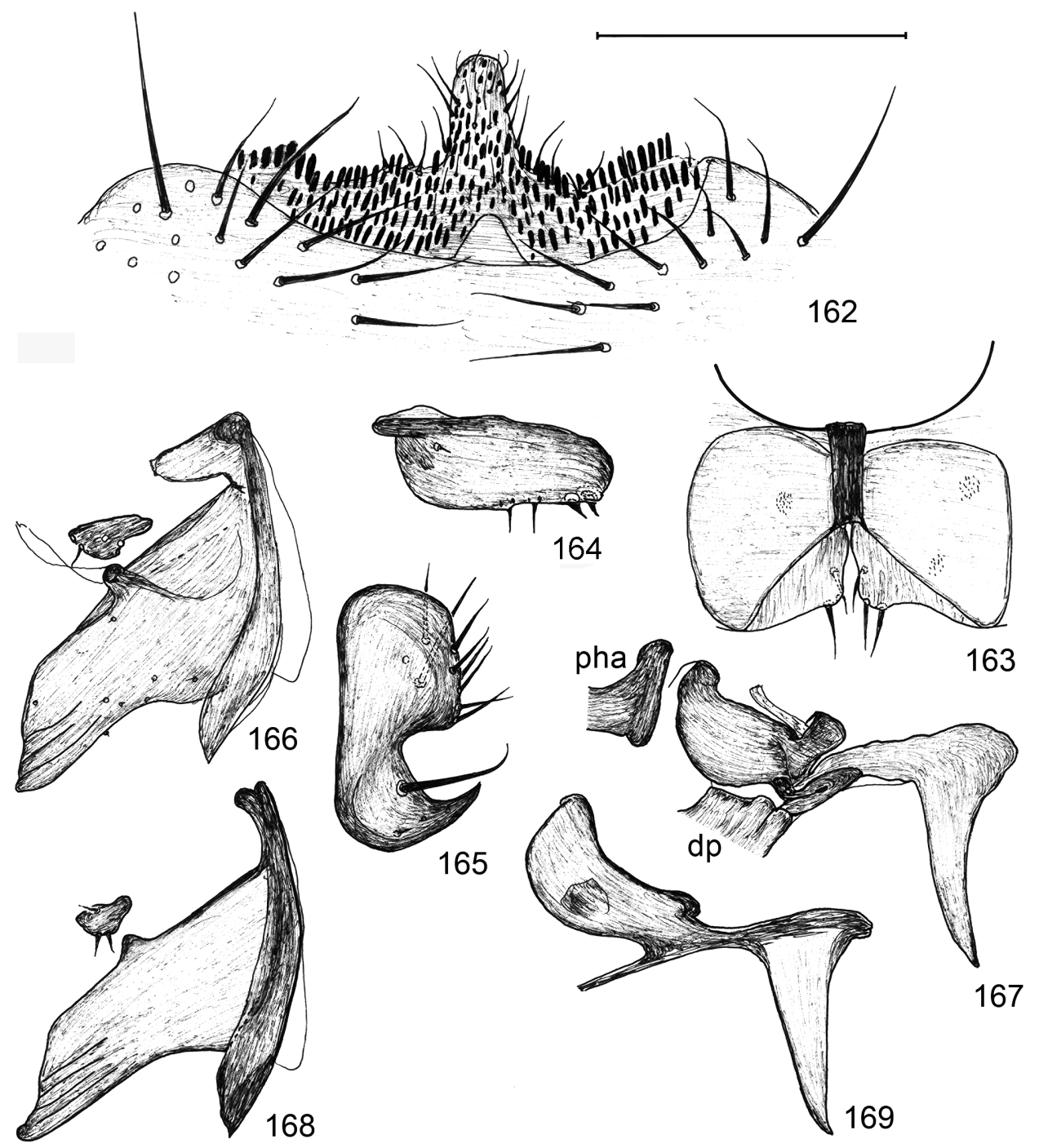

Figs 162-169. 162-167 = Pseudocollinella (Setiopacifrons) nasalis (Richards, 1973), male sternite genitalia. 162 = sternite 5 , ventral view, 163 = cercal part of epandrium and subepandrial sclerite, anterior (inner) view, $164=$ anterior surstylus, broadest (submedial-subventral) view, 165 = posterior surstylus, broadest (sublateral-subcaudal) view, $166=$ postgonite, lateral view, 167 = basiphallus and epiphallus with bases of distiphallus and phallapodeme. $168-169=P$. (Setiopacifrons) sp. from Australia: $168=$ postgonite, lateral view, $169=$ basiphallus and epiphallus, lateral view (dp: distiphallus, pha: phallapodeme). Scale: $0.1 \mathrm{~mm}$ for all. 
by broad black flat pegs. Cercal part of epandrium (Fig. 163) with $2+1$ pairs of setae apicoventrally and on the medial edge. Subepandrial sclerite comparatively long (high), much shorter sagittally. Anterior surstylus (Fig. 164) comparatively long, apex rounded, with short setae only. Posterior surstylus (Fig. 165) with a large curved, apically pointed apical part, with medium-long setae only. Postgonite (Fig. 166) with robust anterior lobe and narrow, subdorsally placed posterior lobe. Anterior lobe of postgonite broad, narrowed in its apical $1 / 5$ with a posterior apex; apical 1/5-1/4 with 3 notches. Basiphallus (Fig. 167) with a dorsal dark sclerite and a long curved epiphallus: basal part directed caudally, its narrow apical part ventrally, with a thin apex.

In the collection of the HNHM there is a male from Australia (Queensland, Townsville, 16-22. III. 1965, Exp. J. Balogh), which although is close to P. (S.) nasalis, is a new species (Figs 168-169). The whole body of the specimen except for its right wing is in a microvial.

\section{P. (Setiopacifrons) paradupliciseta sp. $n$.}

(Figs 170-178)

Holotype male (HNHM): Taiwan: Kaohsiung Hsien, Liukuei, Shan Ping LTER Site over/along a creek, April 2-3, 2003, leg. L. Papp, No. 15.

Paratype male (HNHM, gen. prep.): ibid., Nantou Hsien, Chi Chi TESRI Bot. Garden, 250 m, light traps - Sep 30 - Oct 1, 2000, L. Ronkay, L. Peregovits \& L. Papp, No. 13.

Females with identical or very similar label data: 1 : same as for holotype; 1 : : ibid., along a creek, April 3, No. 19., L. Papp \& M. Földvári; 19: ibid., UV light traps, No. 14, 31.03.-04.04., M. Földvári \& L. Papp; 8 O : same as for paratype.

Measurements in mm: body length 1.94 (holotype), 1.92 (paratype), wing length 1.75 (holotype), 1.72 (paratypes), wing width 0.76 (holotype), 0.76 (paratypes).

Anterior inner orbital seta $0.06 \mathrm{~mm}$, posterior one $0.12 \mathrm{~mm}$. Anterior ifr seta only 0.04 $\mathrm{mm}$. Fore coxa and ventral half of fore femur ochre. None of the last tarsomeres modified. Subbasal costal seta $0.13 \mathrm{~mm}$, other setae on first section $0.09 \mathrm{~mm}$. Second costal section $0.56 \mathrm{~mm}$, 3rd section $0.55 \mathrm{~mm}$. Inter-crossvein section of M1+2 $0.23 \mathrm{~mm}, \mathrm{M}-\mathrm{M}$ crossvein $0.12 \mathrm{~mm}$.

Male sternite 5 is similar to that of $P$. (S.) cederholmi (Fig. 103, see also PAPP 1991: fig. $46)$, i.e. anterior part with a broad central groove and with a pair of blunt black appendage on the sides of the groove; posterior (medial) part with a large caudal sagittal process, without long setae but with hairs on process; caudal edge of the medial part with thick hairs (rather than flat pegs). Cercal part of epandrium (Fig. 171) with a pair of ventral appendages, which bear 2 pairs of setae. Subepandrial sclerite much longer laterally than medially. Hypandrium (Fig. 172) long asymmetrical with long apodeme and long arms. Anterior surstylus (Fig. 173) broad, comparatively short, with several long setae. Posterior surstylus (Figs 174-175) broad-based, with 2 pairs of long setae and a medium-long apical process. Anterior lobe of postgonite (Fig. 176) almost evenly narrow with a small posteriorly directed apex. Posterior lobe of postgonite (Fig. 177) rather broad medially (best seen in a subcaudal-sublateral view). Basiphallus with a caudal swelling (Fig. 178), epiphallus long caudally directed with a thin long basal part joining to basiphallus. 
Female. Since it cannot be distinguished from those of the related species, no female paratype was designated.

Etymology. The specific epithet of this new species refers to its relationship to the species $P$. (S.) dupliciseta (Duda).

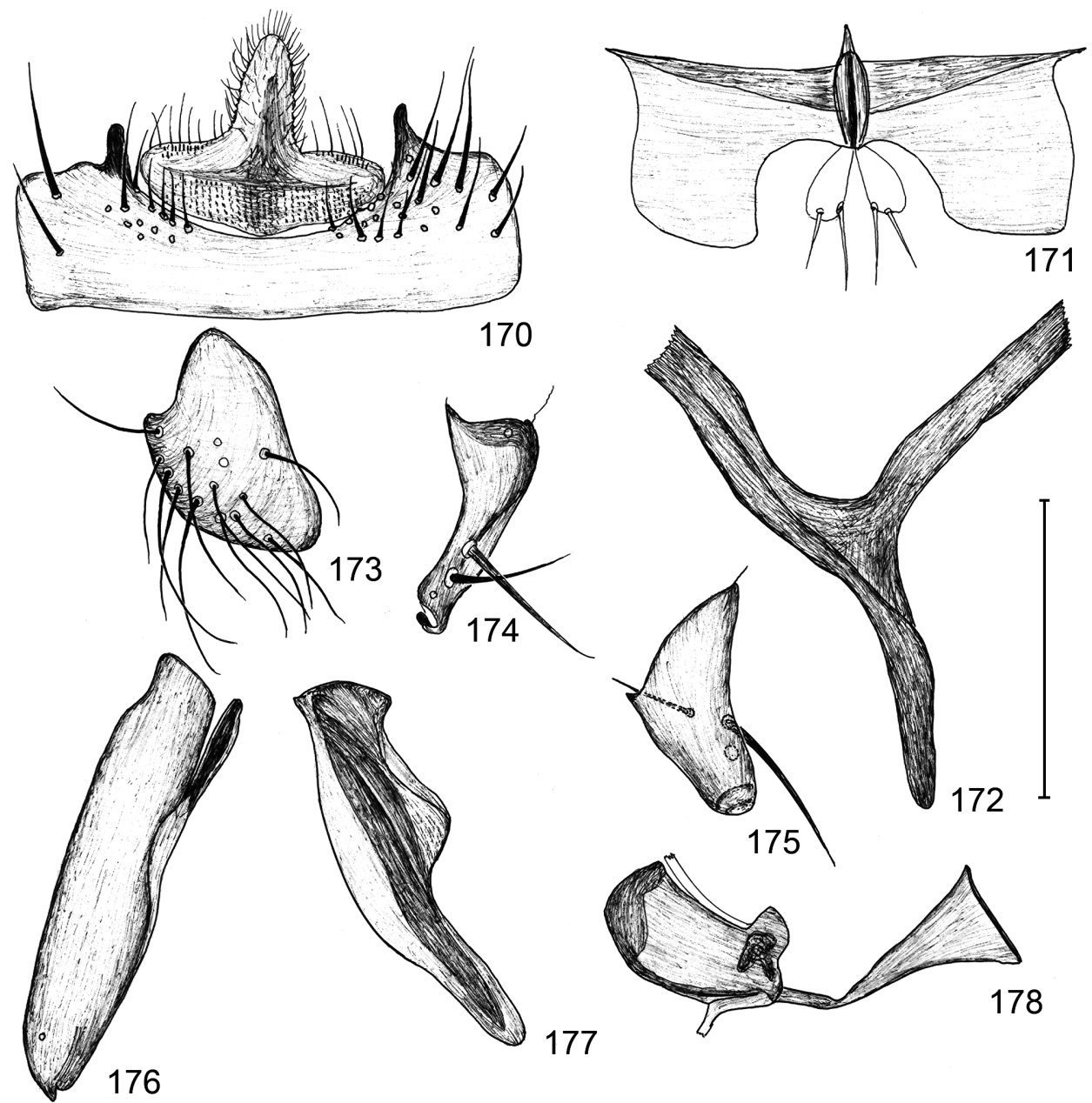

Figs 170-178. Pseudocollinella (Setiopacifrons) paradupliciseta sp. n., male sternite 5 and genitalia. $170=$ sternite 5 , ventral view, $171=$ cercal part of epandrium and subepandrial sclerite, anterior view, 172 = hypandrium, ventral view, 173 = anterior surstylus, broadest view, $174=$ posterior surstylus, subanterior-subventral view, $175=$ posterior surstylus, broadest view, $176=$ anterior part (lobe) of postgonite, broadest (sublateral) view, $177=$ posterior part (lobe) of postgonite, broadest (subcaudal-sublateral) view, $178=$ basiphallus and epiphallus, lateral view. Scale: $0.2 \mathrm{~mm}$ for Figs 170, 172, $0.1 \mathrm{~mm}$ for Figs 171, 173-178. 


\section{P. (Setiopacifrons) pilitibia sp. $\mathrm{n}$. \\ (Figs 179-186)}

Holotype male (HNHM): Thailand: Nan Prov., Ban Na Lae nr Pua, 05. 11. 2004 - over a rocky forest brook, No. 19, L. Papp \& M. Földvári.

Paratype male (HNHM, abdomen and genitalia prepared and preserved in a plastic microvial with glycerol): same as for the holotype.

Measurements in mm: body length 2.30 (holotype), 2.03 (paratype), wing length 2.36 (holotype), 2.14 (paratype), wing width 0.93 (holotype), 0.90 (paratype).

Anterior inner orbital seta c. $1 / 2$ as long as posterior one with 2 additional short setulae. Mid tibia ventrally with long dense thin setae (longest $0.22 \mathrm{~mm}$ ), also fore femur and trochanter are with similar hair-like setae. None of the tarsomeres modified. Subbasal costal seta $0.16 \mathrm{~mm}$, other setae on first section $0.10 \mathrm{~mm}$. Second costal section $0.89 \mathrm{~mm}$, 3rd section at least $0.64 \mathrm{~mm}$ (slightly curved up), inter-crossvein section of M1+2 $0.22 \mathrm{~mm}$, M-M crossvein $0.115 \mathrm{~mm}$.

Male sternite 5 (Fig. 179) comparatively long, without paired caudal processes but anterior part with a deep concave hollow which embraces a thinner plate; however this plate is with a central darker and stronger sclerotised part. Posterior part with minute setulae, anterior part with long setae, mostly in the lateral thirds. Sternite 6 part of synsternite (Fig. 180) with a large dorsally directed plate within the genital vault. Sternite 7 part very large. Hypandrium without apodeme but anterior medial part forms a triangle (Fig. 184). Anterior surstylus (Fig. 181) large with broad base and with short setae only. Posterior surstylus (Figs 182-183) long and narrow with a submedial triangular process and a digitiform apical process in ventral view. In its broadest extension (a sublateral-subventral view) shows two apical lobes: a "cut" one and a digitiform process (the latter with 2 curved setae). Postgonite (Fig. 186) in 2 broadly bifid parts: anterior part narrowed apically with a narrowly rounded apex; posterior part rather broad and less melanised with broadly rounded apex. Phallus peculiar, with a large basiphallus (Fig. 185) whose caudal half is divided into a broader dorsal and a narrow curved ventral part. Epiphallus ventral to basiphallus with a small ventral process. Ejaculatory apodeme small.

Female unknown.

Etymology. The specific epithet of this new species 'pilitibia' (noun) refers to the long dense thin setae on its mid tibia ventrally.

\section{P. (Setiopacifrons) setipuga sp. n.}

(Figs 187-193)

Holotype male (HNHM): Thailand: Trang Prov., Ban Liphang, 16. 11. 2004 - No. 34, over a shadowed, slow brook, leg. L. Papp \& M. Földvári. holotype.

Paratypes (HNHM, 1 male in BMSA): 6 males (2 with gen. prep.): same as for the

Measurements in mm: body length 1.63 (holotype), 1.60-1.71 (paratypes), wing length c. 1.38 (holotype, not precisely measurable), 1.38-1.47 (paratypes), wing width 0.60 (holotype) $0.60-0.68$ (paratypes). 
Frons all dark. Gena broader and dark $(0.09 \mathrm{~mm})$. Only 1 strong inner orbital pair $(0.12 \mathrm{~mm})$, anterior pair only $0.055 \mathrm{~mm}$. Mesonotum with 8 rows of acrostichal microchaetae. None of the tarsomeres is modified. Male mid tibia with black thornlets in the ventral row shorter than in $P$. (S.) communis. Subbasal costal seta $0.11 \mathrm{~mm}$, other seta on first section $0.06 \mathrm{~mm}$. Second costal section $0.46 \mathrm{~mm}$, 3rd section $0.48 \mathrm{~mm}$. Inter-crossvein section
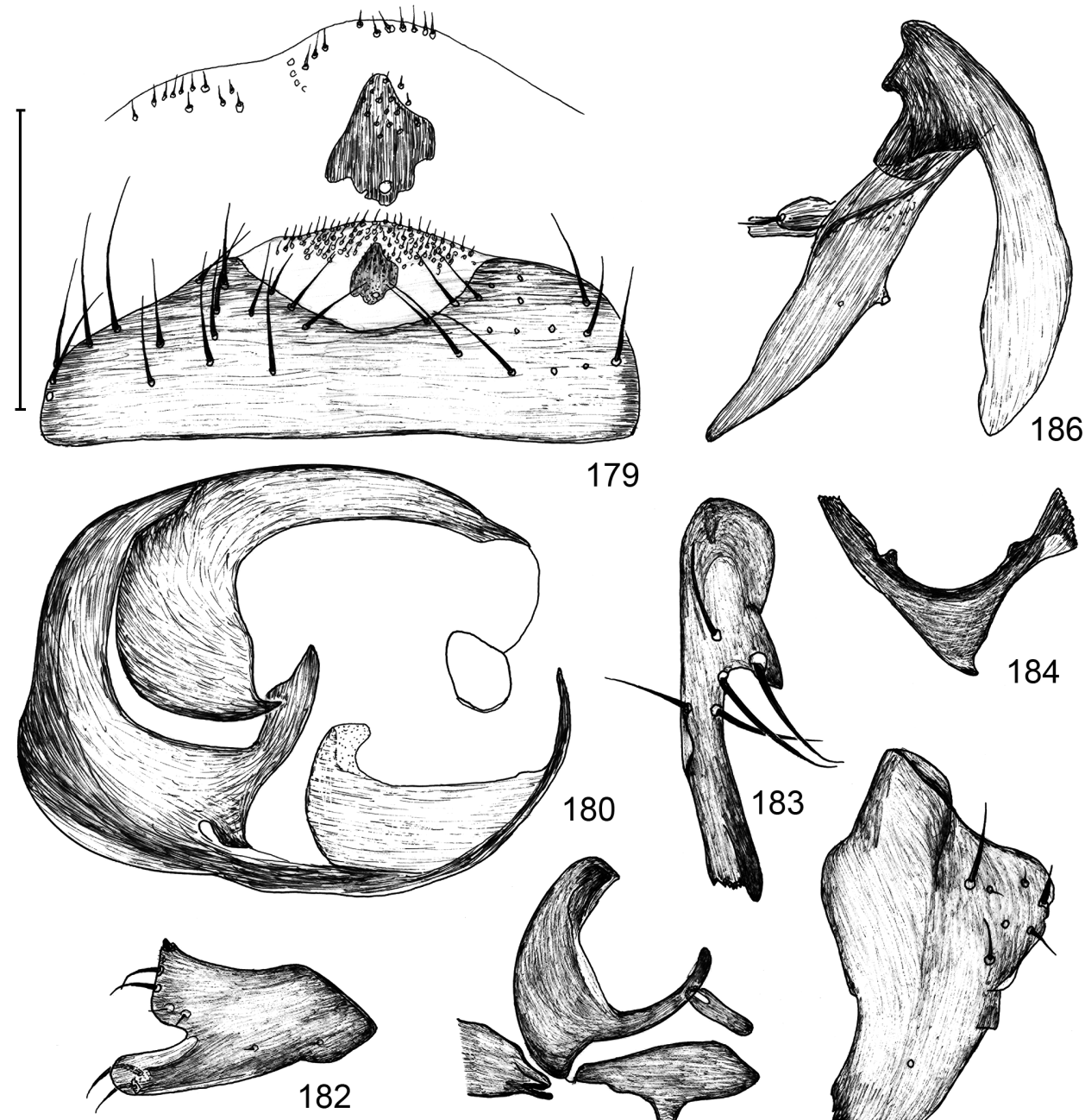

179
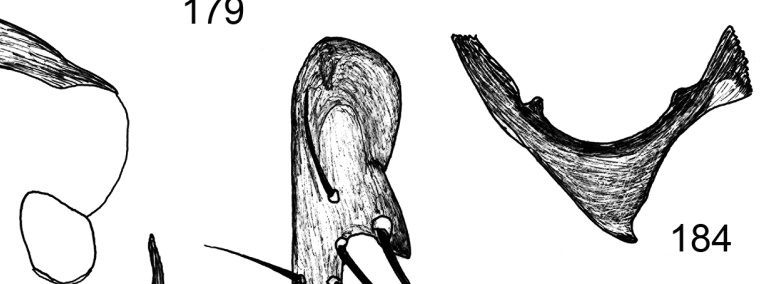

Figs 179-186. Pseudocollinella (Setiopacifrons) pilitibia sp. n., male sternite 5 and genitalia. 179 $=$ sternite 5 , ventral view (outset: its medio-caudal part in higher magnification), $180=$ synsternite, subcaudal view, $181=$ anterior surstylus, broadest (subventral) view, $182=$ posterior surstylus, sublateral-subventral view, 183 = posterior surstylus, ventral view, 184 = hypandrium, ventral view, 185 = basiphallus and epiphallus with base of distiphallus, lateral view, 186 = postgonite in broadest (sublateral) view. Scale: $0.2 \mathrm{~mm}$ for Figs 179-180, 184, 0.1 mm for Figs 181-183, 185-186 and outset of Fig. 179. 
of M1+2 $0.21 \mathrm{~mm}, \mathrm{M}-\mathrm{M}$ crossvein $0.08 \mathrm{~mm}$. Male with numerous very long ventral setae on epandrium.

Male sternite 5 (Fig. 187) is unique: anterior part with a deep medial grove with rounded edges, caudally with numerous long setae. Medial part of sternite 5 (Fig. 188) modified into a curved - rather thin - asymmetrical sclerite, whose caudal margin bears dark thick blunt digitiform processes. Sternite 6 part of synsternite (Fig. 189) extended on the right side with a dorsally curved subtriangular process medially, which forms a

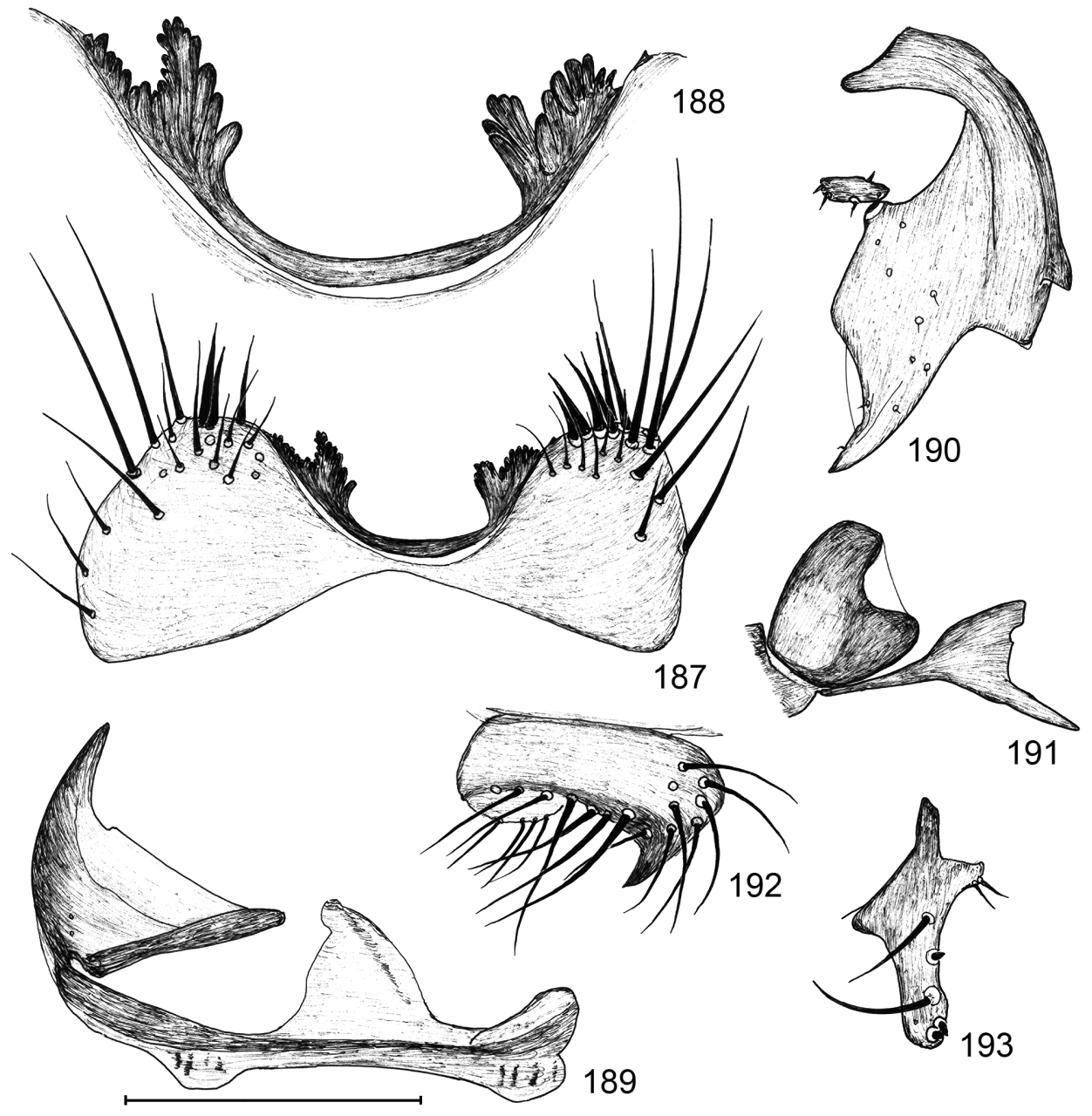

Figs 187-193. Pseudocollinella (Setiopacifrons) setipuga sp. n., male sternite 5 and genitalia. 187 = sternite 5 , ventral view, 188 = sternite 5 , medio-caudal part in higher magnification, ventral view, 189 = sternites 6 and 7 part of synsternite, subventral-subcaudal view, $190=$ postgonite, broadest (sublateral) view, 191 = basiphallus and epiphallus, lateral view, $192=$ anterior surstylus, broadest (subventral-sublateral) view, $193=$ posterior surstylus, broadest (anterior subventral) view. Scale: 0.2 mm for Figs 187, 189, 0.1 mm for Figs 188, 190-193. 
part of the concave genital vault (pouch); sternite 7 comparatively small with thickened anterior margin towards the process of sternite 6 part. Anterior surstylus (Fig. 192) with a sharp medial process, long setae concentrated on its caudal and medial part. Posterior surstylus (Fig. 193) broad basally narrow in its apical half with 2 longer setae and 3 short
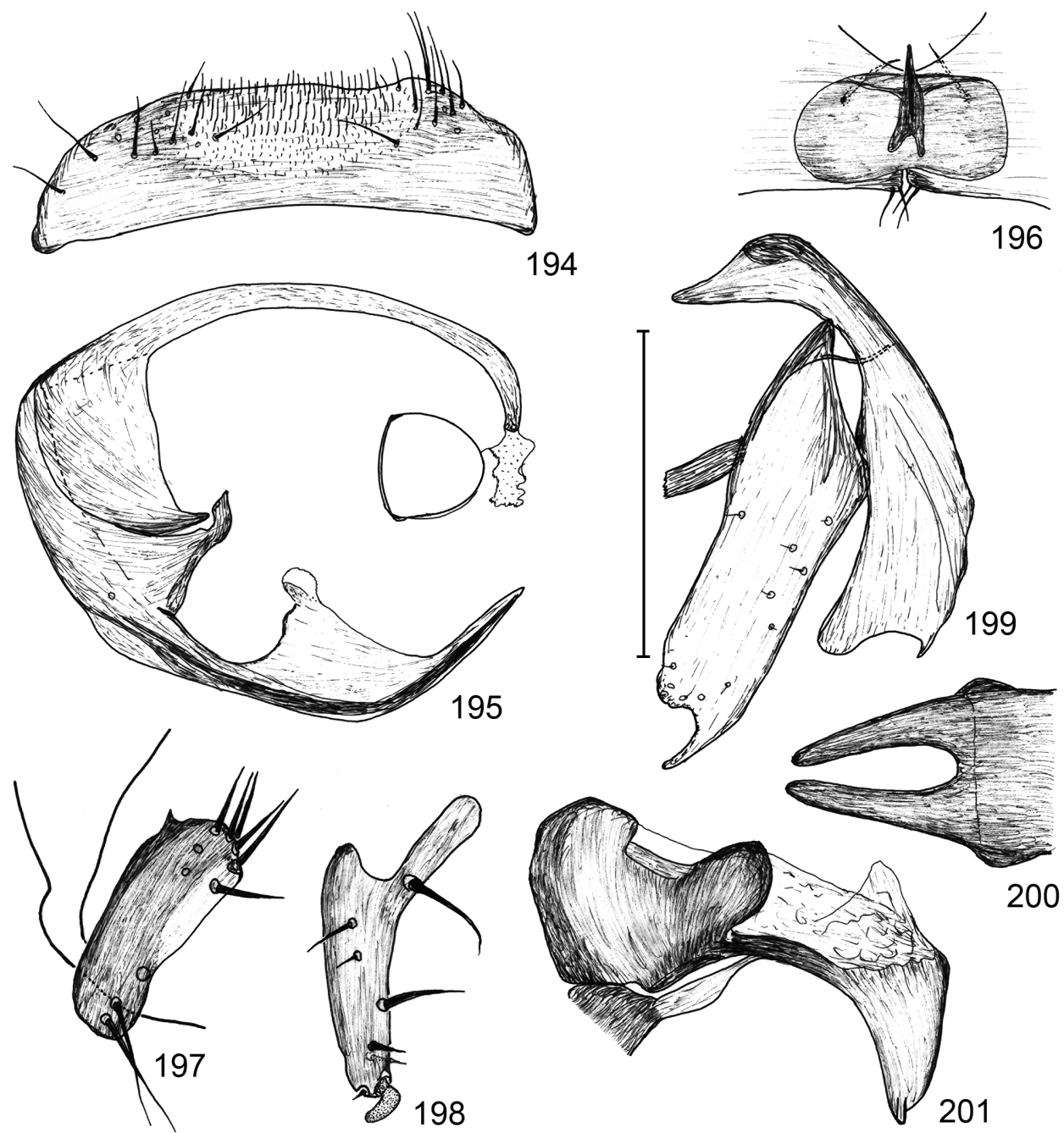

Figs 194-201. Pseudocollinella (Setiopacifrons) simplicisternum sp. n., male sternite 5 and genitalia. 194 = sternite 5, ventral view, $195=$ synsternite, subcaudal view, $196=$ cercal part of epandrium and subepandrial sclerite, ventral (!) view, 197 = anterior surstylus, broadest (subventral-submedial) view with borders of epandrium and hypandrium, $198=$ posterior surstylus, broadest (subanterior) view, $199=$ postgonite in broadest (sublateral) view, $200=$ base of distiphallus, ventral-caudal view, 201 = basiphallus and epiphallus with basal part of distiphallus, lateral view. Scale: $0.2 \mathrm{~mm}$ for Figs 194-196, $0.1 \mathrm{~mm}$ for Figs 197-201. 
thorns. Postgonite (Fig. 190) is very characteristic: anterior lobe and posterior (more basal) lobe seem to be fused most caudally (very broad there), apical third of anterior lobe much narrowed with an anteriorly directed sharp apex. Basiphallus (Fig. 191) dorsally extended both anteriorly and posteriorly, epiphallus long, caudally directed with a thin sharp caudal process and a thin anterior process, which reaches distiphallus.

Female. Since it cannot be distinguished from those of the related species, no female paratype was designated.

Etymology. The specific epithet of this new species is from the Latin seti- (setose) and puga (buttocks), referring the strongly setose ventral part of epandrium of the species.

\section{P. (Setiopacifrons) simplicisternum sp. n. (Figs 194-201)}

Holotype male (HNHM, gen. prep.): Taiwan: Kaohsiung Hsien, Liukuei, Shan Ping LTER Site- creek valley, No. 13, Mar 31-April 1, 2003, leg. L. Papp \& M. Földvári.

Measurements in mm: body length 1.70 (holotype), wing length 1.53, wing width 0.69 . First interfrontal seta anterior to the 3 stronger ones is only $0.04 \mathrm{~mm}$ long. Mesonotum with 8 rows of acrostichal microchaetae. None of the last tarsomeres modified. Subbasal costal seta $0.15 \mathrm{~mm}$, other setae of the section $0.09 \mathrm{~mm}$. Second costal section $0.52 \mathrm{~mm}, 3 \mathrm{rd}$ section $0.51 \mathrm{~mm}$. Inter-crossvein section of M1+2 $0.20 \mathrm{~mm}, \mathrm{M}-\mathrm{M}$ crossvein $0.11 \mathrm{~mm}$.

Male sternite 5 (Fig. 194) rather short and simple: medial part slightly less sclerotised (melanised), there with numeous short setulae, its longer setae are rather sparse. Sternite 6 part of synsternite (Fig. 195) with rather large - apically narrowed - medial lobe, which emerges dorsally into the genital vault. Sternite 7 part comparatively small with a short curved process on its right side. Sternite 8 part broad but short. Cercal part of epandrium (Fig. 196) with 2 pair of small setae in the sagittal corner. Subepandrial sclerite small and laterally rounded. Anterior surstylus (Fig. 197) comparatively small with several mediumlong setae on its margin. Posterior surstylus (Fig. 198) long with a long narrow basal part, with several medium-long setae and a broad rounded apical process. Postgonite (Fig. 199) in 2 super-positioned parts: anterior part long and broad with a subapical emargination and with a slightly curved narrow apex. Posterior part of postgonite broadened subapically, with 2 apices: a blunt anterior and a short sharp posterior apex. Basiphallus (Fig. 201) rather large, epiphallus joins to basiphallus through a long narrow process, whose basal part joins to base of distiphallus by an even thinner process. Dorsal part of epiphallus membranous. Distiphallus (Fig. 200) terminates in a long fork, when seen subventrally.

Figs 202-211. Pseudocollinella (Setiopacifrons) tercia sp. n., male sternite 5 and genitalia. $202=$ sternite 5, ventral view, 203 = sternite 5, medio-caudal part in higher magnification, ventral view, $204=$ synsternite, caudal view, $205=$ ventral part of synsternite, ventral view, $206=$ anterior surstylus, broadest (subventral) view, 207 = hypandrium, ventral view, $208=$ posterior surstylus, anterior ventral view, $209=$ basiphallus and epiphallus with basal part of distiphallus, lateral view, $210=$ anterior part (lobe) of postgonite in broadest (sublateral) view, 211 = basal part of postgonite, dorsal view. Scale: $0.2 \mathrm{~mm}$ for Figs 202, 204-205, 207,

$0.1 \mathrm{~mm}$ for Figs 203, 206, 208-211. 
Female unknown.

Etymology. The specific epithet (noun) of the new species refers to the very simple and shortly setose medial part of male sternite 5 .
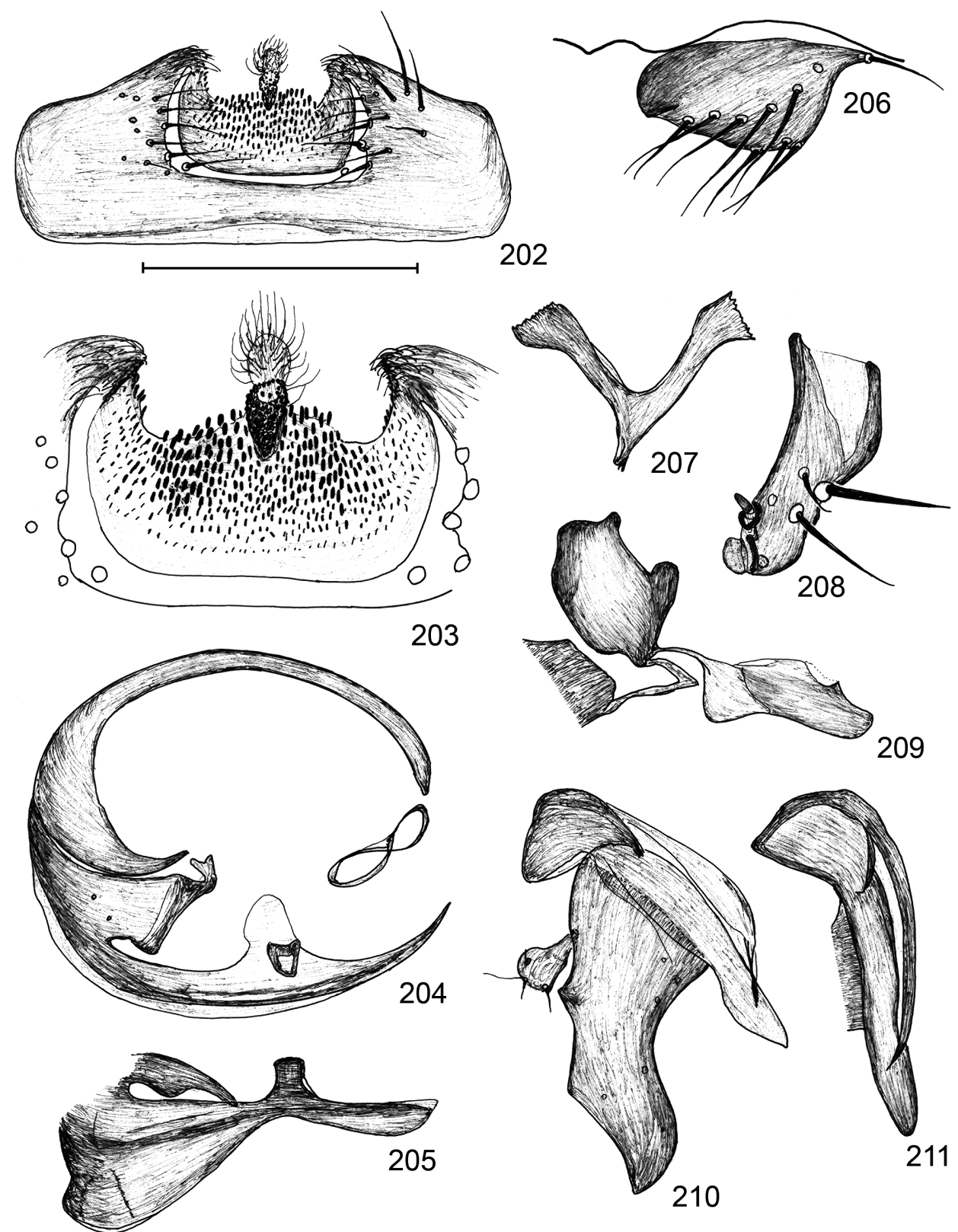


\section{P. (Setiopacifrons) tercia sp. $n$.}

(Figs 202-211)

Holotype male (HNHM, gen. prep.): Thailand: Mae Fang N.P., over \& along a forest brook - Nov 1, 2004, No. 14, L. Papp \& M. Földvári.

Paratype male (HNHM, gen. prep): Thailand: Nan Prov., Ban Na Lae nr. Pua, 05. 11.'04 - over a rocky forest brook, No. 19, L. Papp \& Földvári.

Measurements in mm: body length 2.31 (holotype), 2.25 (paratype), wing length 2.30 (holotype), 2.26 (paratype), wing width 0.98 (holotype), 0.96 (paratype).

Anterior inner orbital seta $0.05 \mathrm{~mm}$, the posterior one $0.09-0.10 \mathrm{~mm}$. In addition of the 2 long dorsocental setae, 1 presutural and 1 postsutural short dc setae discernible. Hind last tarsomere modified, claws straight and dorsally placed. Subbasal costal seta $0.19 \mathrm{~mm}$, other setae on first section $0.11 \mathrm{~mm}$. Second costal section $0.79 \mathrm{~mm}$, 3rd section $0.69 \mathrm{~mm}$. Inter-crossvein section of M1+20.32, M-M crossvein $0.14 \mathrm{~mm}$.

Male sternite 5 (Fig. 202) is spectacular: anterior (lateral) part with a broad medial groove, medial borders of this groove lengthened apical part of this extension with some small thorns. Longer setae of anterior part emerge caudally and on sides of groove, the latter ones are medio-clinate. Posterior (medial) part of sternite 5 (Fig. 203) with rounded caudal sagittal hairy process, caudal margin and almost all surface of medial part are covered by flat blunt black pegs. Synsternite (Figs 204-205) with short sternite 6 and 8 parts, sternite 6 part with a medial process, better seen in ventral view (Fig. 205); medial margin of sternite 7 part thickened. Hypandrium (Fig. 207) with short apodeme, or rather, apical (medial) part of hypandrium forms a triangle. Anterior surstylus (Fig. 206) broad but sharply narrowed caudally with c. 10 long setae. Posterior surstylus (Fig. 208) with 2 subapical thorn and comparatively broad apical process: among its setae with a submedial very thick seta. Postgonite (Figs 210-211) robust, anterior lobe edged in its apical third, posterior lobe (Fig. 211) with a broadened anterior part; dorsally with a less sclerotised stripe. Basiphallus (Fig. 209) compact with anterior and posterior swellings dorsally, epiphallus caudally directed, apically rounded, and connected to the basiphallus through a very narrow sclerite.

Female unknown.

Etymology. The specific epithet of this new species 'tercia' (Latin: third) refers to the modified hind last tarsomere of the species.

\section{P. (Setiopacifrons) trifida sp. $\mathrm{n}$.}

(Figs 212-220)

Holotype male (HNHM, gen. prep.): Taiwan: Taipei, Nanshih Chiao, Ha Lo-Da, 450, L. Papp \& L. Ronkay - rocky forest undergrowth, Oct 10, 2000, No. 21.

Measurements in mm: body length 1.56 (holotype), wing length 1.44 (not precisely measureable, strongly downcurved), wing width 0.65 .

Anterior inner orbital seta $0.06 \mathrm{~mm}$, posterior one $0.09 \mathrm{~mm}$, anterior interfrontal seta $0.04 \mathrm{~mm}$ only. Acrostichal microchaetae in 8 rows. None of the last tarsomeres modified. 
Subbasal costal seta $0.12 \mathrm{~mm}$, other setae on first section $0.06 \mathrm{~mm}$. Third costal section definitely longer than second section: second section c. $0.38 \mathrm{~mm}$, third section $0.44 \mathrm{~mm}$. Inter-crossvein section $0.16 \mathrm{~mm}, \mathrm{M}-\mathrm{M}$ crossvein $0.08 \mathrm{~mm}$.
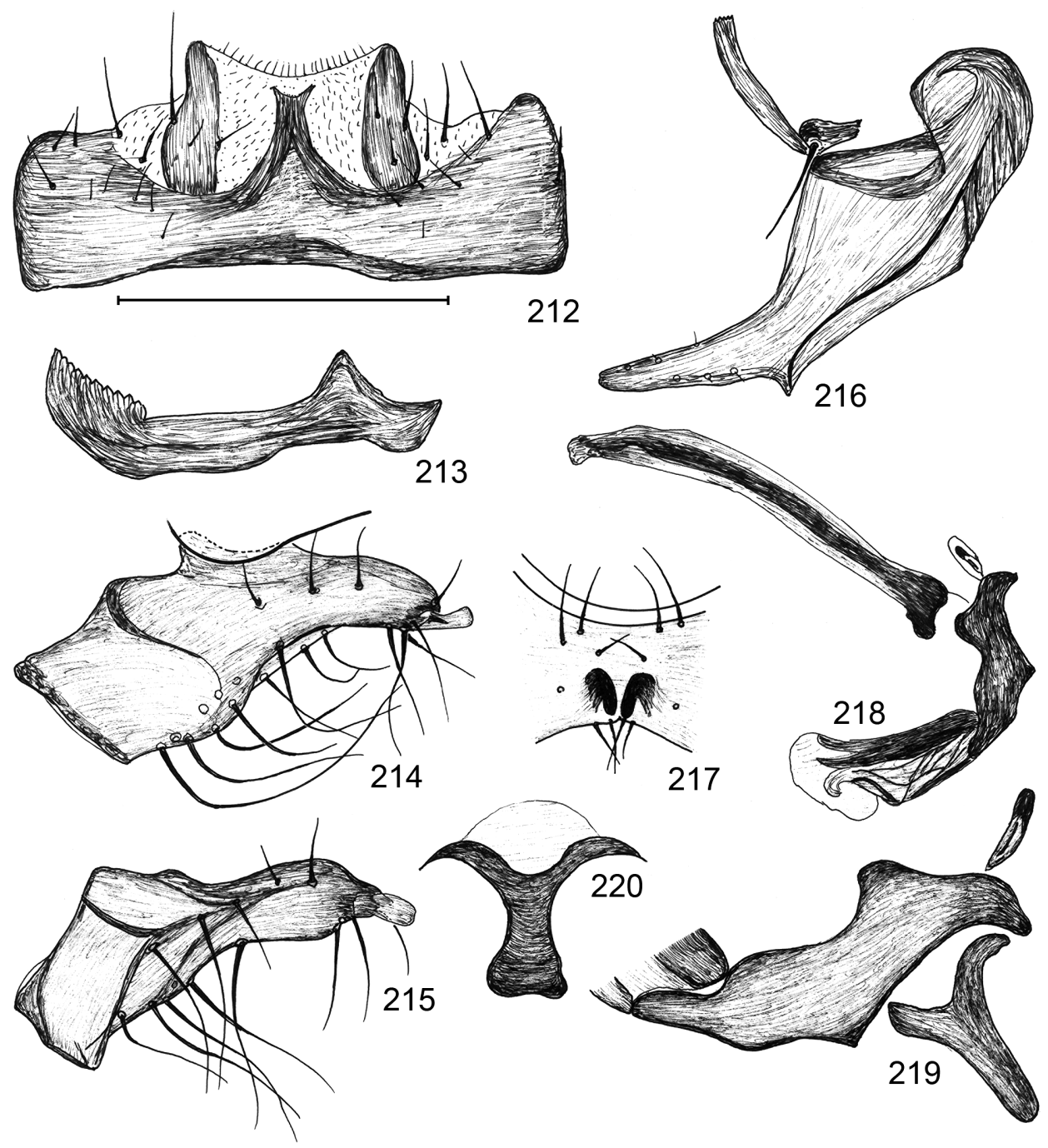

Figs 212-220. Pseudocollinella (Setiopacifrons) trifida sp. n., male sternite 5 and genitalia. 212 = sternite 5 , ventral view, 213 = ventral part of synsternite, true ventral view, $214=$ surstylus, broadest (subventral-sublateral) view, 215 = surstylus, ventral view, 216 = postgonite, broadest (sublateral) view, $217=$ cercal part of epandrium, caudal view, posterior surstylus, broadest (subanterior) view, 218 = basiphallus (without epiphallus), distiphallus and phallapodeme with ejaculatory apodeme, 219 = basiphallus and epiphallus with base of distiphallus and ejaculatory apodeme, $220=$ epiphallus, ventral view. Scale: $0.2 \mathrm{~mm}$ for

Figs 212-213, 217-218, 0.1 mm for Figs 214-216, 219-220. 
Male sternite 5 (Fig. 212) on its medial (caudal) part with 3 dark stripes: 1 sagittal and 2 lateral ones. Anterior broader part of sternite 5 with a pair of "hollows" (i.e. not a single groove) and with a sagittal, better sclerotised, apically slightly concave process, which is actually fused to the medial edges of the posterior part. Posterior part of sternite 5 with a pair of darker and stronger sclerotised longish plates; apex membranous with minute setulae. Long setae on sternite 5 sparse. Synsternite: sternite 6 part (Fig. 213) without dorsally directed lobes medially, but sternite 6 narrow and dark lengthened to the right side with a swelling on that right part. No hypandrial apodeme. Cercal part of epandrium (Fig. 217) subventrally with a pair of broad-based, ventrally curved blunt black processes. Subepandrial sclerite small. Surstylus not divided (Figs 214-215), or rather anterior and posterior parts fused; anterior part very broad, medial margin with very long hair-like setae, caudal apex with the "usual" broad flat caudal process (of the posterior surstylus in other species). Postgonite (Fig. 216) not divided, i.e. a single postgonite present, in lateral view with a long narrow, narrowly rounded apical part; in its broadest extension one can detect its strong caudal process on the border of its apical and medial parts. Basiphallus (Figs 218-219) forms a long sclerite, which is as long as distiphallus (Fig. 218). Epiphallus (Figs 219-220) broad-based, ventrally lengthened and apically rounded. Ejaculatory apodeme thin with a broader coat.

Female unknown.

Etymology. The specific epithet of this new species (Latin 'trifida') means three branches, referring to the medial part of its male sternite 5 , where one can see 3 dark stripes.

\section{P. (Setiopacifrons) vietnamensis sp. $\mathrm{n}$.}

(Figs 221-229)

Holotype male (HNHM, gen. prep.): Vietnam: O-qui-ho, 26. IX. 1963, leg. T. Pócs. The holotype is slightly damaged, mesonotal and scutellar setae all broken off.

Paratypes (HNHM): 1 male (gen. prep.): Nay lam, NE of Hanoi - 14.IV. 1966., leg. Topál - "Pseudocollinella sp. n.? J" J. Roháček det. 2000; 1 female: Sa-pa, fényre repült [on light], 1963. IX. 22., leg. T. Pócs - “Opacifrons q dupliciseta Duda" det. L. Papp 1989. 1 female: Vietnam, Hông-gai - fénycsapda [light trap] - 1963. IX. 8., leg. T. Pócs.

Measurements in mm: body length 1.75 (holotype), 1.70-1.80 (paratypes), wing length 1.53 (holotype), 1.50-1.62 (paratypes), wing width 0.64 (holotype) $0.62-0.66$ (paratypes).

Anterior inner orbital seta $0.08 \mathrm{~mm}$, posterior one $0.10 \mathrm{~mm}$. None of the last tarsomeres modified. Subbasal costal seta $0.15 \mathrm{~mm}$, other setae on first section $0.08-0.09 \mathrm{~mm}$. Second costal section $0.49 \mathrm{~mm}$, 3rd section $0.51 \mathrm{~mm}$. Inter-crossvein section of M1+2 0.24 $\mathrm{mm}, \mathrm{M}-\mathrm{M}$ crossvein $0.08 \mathrm{~mm}$.

Male sternite 5 (Fig. 221) peculiar with 2 pairs of thick, apically curved thorns. Anterior part of sternite 5 subquadratic with comparatively small medio-caudal hollow. Thorns emerge on the edge of that area: anterior pair closer to each other on anterior edge, posterior pair farther from each other on the posterior edge. The posterior part of sternite 5 is somewhat less sclerotised and melanised with numerous short and blunt setulae and with a narrow sagittal lobe. Longer setae of sternite 5 part emerge on its lateral-caudal part. Sternite 6 part of synsternite (Fig. 222) thick and black, ventrally with a moderately large 
dorsally directed lobe (Fig. 222); sternite 7 part with broad rightwards directed apex, sternite 8 part comparatively short but broad. Hypandrium (Fig. 226) with thin apodeme and broad arms. Anterior surstylus (Fig. 223) membranous medio-basally, with extremely long setae. Posterior surstylus (Figs 224-225) comparatively small with rounded apex and with 2 extremely thick thorns; its apical process rather small. Postgonite in 2 broadly divided parts (Fig. 227): anterior part broad-based with much narrower medial part and with a blunt apex; posterior part comparatively broad, slightly curved with a small anterior apex.

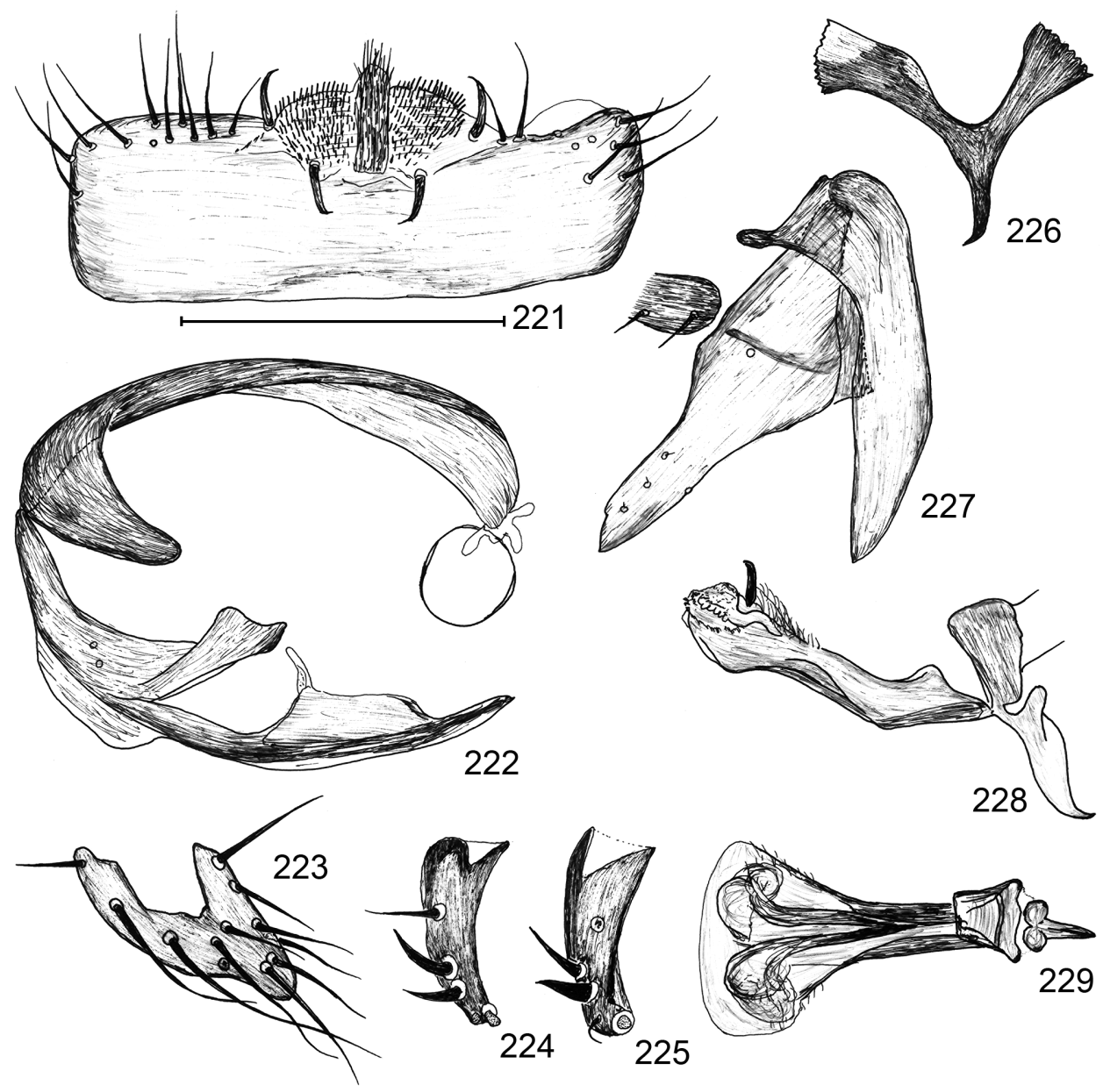

Figs 221-229. Pseudocollinella (Setiopacifrons) vietnamensis sp. n., male sternite 5 and genitalia. 221 = sternite 5, ventral view, 222 = synsternite, subcaudal view (perpendicular to sternite 7 part), 223 = anterior surstylus, broadest (subventral) view, $224=$ posterior surstylus, ventral view, $225=$ posterior surstylus, broadest (subanterior) view, $226=$ hypandrium, ventral view, 227 = postgonite, lateral view, 228 = phallus, lateral view, 229 = phallus ven-

tral view. Scale: 0.2 mm for Figs 221-222, 226, 228-229, $0.1 \mathrm{~mm}$ for Figs 223-225, 227. 
Basiphallus (Fig. 228) comparatively small and compact with a large epiphallus; epiphallus broad with backwards directed apex. Distiphallus (Fig. 229) strongly broadened in its apical half; distiphallus with a dorsal subapical process and with hairs on apical dorsal half (Fig. 228).

Female. It cannot be distinguished from the female of $P$. (S.) formosensis; the paratypes were designated as such based on the localities only. nam).

Etymology. The specific epithet of the new species refers to its type locality (Viet-

\section{P. (Setiopacifrons) sp.}

1 male (HNHM): Taiwan: Ilan Hsien, Fu-Shan LTER Site, Sep 26, 2000, leg. L. Papp, No. 7 - lake-shore vegetation and along a brook bed. This specimen belongs to all probability to a new species close to $P$. (S.) vietnamensis.

In the HNHM there are eight females from Taiwan and 61 females from Thailand, which have been left unidentified.

\section{Key to the extra-Afrotropical species of the subgenus Setiopacifrons (males)}

1. Male sternite 5 with a caudal sagittal lobe (e.g. Figs 103, 124).

- $\quad$ Male sternite 5 without a caudal sagittal lobe (e.g. Figs 153, 188, 194). 7

2. Anterior (lateral part) of sternite 5 without a pair of black processes in the caudal border of the central groove (Figs 112, 124, 162).

- Anterior (lateral part) of sternite 5 with a pair of blunt black processes in the caudal border of the central groove (Figs 103, 143, 170).

3. Medio-caudal part (incl. sagittal lobe) covered by dense flat black pegs (Fig. 162). Anterior surstylus with short setae only (Fig. 164). Posterior surstylus with a large sharp apical process (Fig. 165). Anterior lobe of postgonite very broad (Fig. 166). Epiphallus (Fig. 167) with a large dorsal hump medially and a ventrally directed apical part. Australia. P. (S.) nasalis (Richards)

- Medio-caudal part of sternite 5 with hair-like setae, only partly covered by minute black pegs (Figs 112, 124). Anterior surstylus with long setae (Figs $119,127)$. Posterior surstylus broadly rounded apically without a large sharp apical process (Figs 120,128). Anterior lobe of postgonite very broad (Fig. 129). Epiphallus broadening caudally in profile (e.g. Fig. 122). 
4. Ventral part of epandrium with numerous long setae (Fig. 123). Caudal sagittal process of sternite 5 broader (Fig. 124) incl. its darker sagittal part; medio-caudal part with thin hairs only. Posterior surstylus longer and otherwise shaped (Fig. 128). Anterior lobe of postgonite (Fig. 129) broader with a blunt apex. Taiwan. $\quad P$. (S.) dupliciseta (Duda)

- Ventral part of epandrium with much less long setae (Fig. 114). Caudal sagittal process of sternite 5 narrower (Fig. 112), particularly so for its darker sagittal part; medio-caudal part with thickened hairs. Posterior surstylus shorter (Fig. 120). Anterior lobe of postgonite (Fig. 116) narrowed in apical half with a distinct anterior apex. Thailand.

P. (S.) communis sp. $\mathrm{n}$.

5. Caudal sagittal lobe of sternite 5 broader, caudal process of its anterior part broader, not digitiform (Fig. 143). Anterior sustylus with a medial edge (Fig. 145). Medio-caudal edge of cercal part of the epandrium with thick setae (Fig. 151). Anterior lobe of postgonite narrow (Fig. 149). Hypandrial apodeme less long (Fig. 148). Japan (Kyushu).

P. (S.) japonica sp. n.

- Caudal sagittal lobe of sternite 5 narrower (Figs 103, 170), caudal process of its anterior part narrower, digitiform. Anterior surstylus normal or broad but with out medial edge. Medio-caudal edge of cercal part of epandrium with thin setae. Anterior lobe of postgonite broad (Figs 107, 176). Hypandrial apodeme very long (Fig. 172).

6. Posterior margin of the medial part of sternite 5 (Fig. 170) with minute pegs only. Caudal sagittal lobe of sternite 5 less long. Anterior surstylus with thin setae (Fig. 173). Anterior lobe of postgonite evenly broad with a very small apex (Fig. 176). Taiwan.

P. (S.) paradupliciseta sp. n.

- Posterior margin of the medial part of sternite 5 (Fig. 103) with longer narrow pegs. Caudal sagittal lobe of sternite 5 longer (Fig. 103). Anterior surstylus with thick setae (Fig. 109). Anterior lobe of postgonite very broad in its basal half, apical 1/8-1/7 narrowed with a larger blunt apex (Fig. 107). Sri Lanka.

P. (S.) cederholmi (L. Papp)

7. Epandrium with long dense thick setae. Sternite 5 deeply concave (Fig. 187), medio-caudal part with a curved - rather thin - asymmetrical sclerite (Fig. 188), whose caudal margin bears dark thick blunt digitiform processes. Postero-lateral part of sternite 5 lengthened and rounded with numerous long setae (conspicuous also without preparation). Thailand.

P. (S.) setipuga sp. n. 
- $\quad$ Epandrium with normal setae, though they may be numerous. Sternite 5 simple or convex with specific structures.

8. Medio-caudal part of sternite 5 simple (Fig. 194) or convex with minute hairs (Figs 153, 179). Postgonite broadly bifid.

- Medio-caudal part of sternite 5 convex with specific structures (e.g. Fig. 221).

9. Sternite 5 simple, not divided, medio-caudal part only with short hairs (Fig. 194). Anterior surstylus (Fig. 197) normal. Mid tibia ventrally without long hair-like setae. Taiwan.

P. (S.) simplicisternum sp. n.

- $\quad$ Sternite 5 strongly convex medio-caudally (Fig. 179). Anterior surstylus modified.

10. Medio-caudal part of sternite 5 with minute hairs and a dark plate (Fig. 179). Anterior surstylus large and broad-based (Fig. 181). Basiphallus with 2 long dorsal processes. Epiphallus shorter with a smaller ventral process (Fig. 185). Mid tibia ventrally with long hair-like setae. Thailand.

P. (S.) pilitibia sp. n.

- Medio-caudal part of sternite 5 with longer thick hairs but without a dark plate (Fig. 153). Anterior surstylus with strong setae on caudal margin (Fig. 159). Basiphallus in profile (Fig. 161) with 2 dorsal swellings only. Epiphallus (Fig. 161) caudally directed with a caudal apex. Mid tibia ventrally without long hair-like setae. N Korea.

P. (S.) koreana sp. n.

11. Anterior part of sternite 5 with 2 broad processes, each with stronger sclerotisation and melanisation (Fig. 212). Sagittal part separating grooves also darker and stronger sclerotised. Taiwan.

P. (S.) trifida sp. n.

- Anterior part of sternite 5 with a single groove, posterior central part is spectacular (Figs 133, 203, 221).

12. Medial part of sternite 5 with 2 pairs of curved thick thorns (Fig. 221), medio-caudal part with numerous hairs (no black flat pegs). Anterior lobe of postgonite narrow (Fig. 227).

P. (S.) vietnamensis sp. $n$.

- Medial part of sternite 5 with a caudal sagittal lobe, which has a thickened base and long hairs apicaly and laterally (e.g. Fig. 133). Body of the medial part covered by flat black pegs (Figs 133, 203). Anterior lobe of postgonite broad (Figs 141, 210). 
13. Caudal sagittal lobe of sternite 5 narrower (Fig. 133). Apical $1 / 3$ of anterior lobe of postgonite broader (Fig. 141). Medial seta of posterior surstylus not thick. Hind last tarsomere not modified. Taiwan.

P. (S.). formosensis sp. n.

- Caudal sagittal lobe of sternite 5 broader (Fig. 203). Apical 1/3 of anterior lobe of postgonite narrower (Fig. 210). Medial seta of posterior surstylus extremely thick. Hind last tarsomere modified but see page 37. Thailand.

P. (S.) tercia sp. n.

\section{CLOSING REMARKS}

In summary, two species of the formerly described ones, Opacifrons cederholmi L. Papp, 1991 and Leptocera (Opacifrons) dupliciseta Duda, 1925 are transferred to the subgenus P. (Setiopacifrons). Within Pseudocollinella Duda, 1924, Leptocera (Pseudocollinella) nasalis Richards, 1973 is relegated to the new subgenus. The relegation is still questionable but probable for Leptocera (Pseudocollinella) difficilis Richards, 1973. As for the obscure Bispinicercia liupanensis Su et Liu, 2009, the proposal of Marshall et al. (2011) is accepted, that name is a junior subjective synonym of Opacifrons Duda, 1918 and its type species is close to O. coxata (Stenhammar, 1855).

\section{REFERENCES}

Carles-Tolrá, M. (1990) New species and records of Sphaeroceridae (Diptera) from Spain. The Entomologist's Monthly Magazine 126: 33-46.

Duda, O. (1925) Die aussereuropäischen Arten der Gattung Leptocera Olivier - Limosina Macquart (Dipteren) mit Berücksichtigung der europäischen Arten. Archiv für Naturgeschichte 90A(1924): 5-215.

Marshall, S. A. \& Langstaff, R. (1998) Revision of the New World species of Opacifrons Duda (Diptera, Sphaeroceridae, Limosininae). Contributions in Science, Natural History Museum of Los Angeles County 474: 1-27.

Marshall, S. A. \& Sмith, I. P. (1993) A revision of the Nearctic Pseudocollinella Duda (Diptera; Sphaeroceridae). Canadian Journal of Zoology 71: 835-857. doi 10.1139/z93-109

Marshall, S. A., RoháčeK, J., Dong, H. \& Buck, M. (2011) The state of Sphaeroceridae (Diptera: Acalyptratae): a world catalog update covering the years 2000-2010, with new generic synonymy, new combinations, and new distributions. Acta Entomologica Musei Nationalis Pragae 51(1): 217-298.

PApp, L. (1974) New species and records of Sphaeroceridae from Central Asia (Diptera). Annales historico-naturales Musei nationalis hungarici 66: 251-268.

PAPP, L. (1977) Sphaeroceridae (Diptera) from Tunisia. Folia entomologica hungarica, S.N., 30(2): 119-123. 
PAPP, L. (1979) New species and records of Sphaeroceridae (Diptera) from the USSR. Annales historico-naturales Musei nationalis hungarici 71: 219-230.

PApP, L. (1991) Oriental Limosininae: new species and records (Diptera, Sphaeroceridae). Acta Zoologica Academiae Scientiarum Hungaricae 37(3-4): 225-251.

PApp, L. (2012) Five new Afrotropical species of Opacifrons (Diptera, Sphaeroceridae). Acta Zoologica Academiae Scientiarum Hungaricae 58(2): 121-143.

PApp, L. \& Roháček, J. (2016) 99. Sphaeroceridae (Lesser dung flies). In: Kirk-Spriggs, A. (ed.): Manual of the Afrotropical Diptera, Vol. 2. [in press]

Richards, O. W. (1929) Systematic notes on the Borboridae (Diptera) with description of new species of Leptocera (Limosina). The Entomologist's Monthly Magazine 65: 171-176.

Richards, O. W. (1973) The Sphaeroceridae (= Borboridae or Cypselidae; Diptera Cyclorrhapha) of the Australian Region. Australian Journal of Zoology, supplementary series, 22: 297-401. doi 10.1071/AJZS022

RонÁčEK, J. (1975) Leptocera (Opacifrons) moravica sp.n. from Czechoslovakia (Diptera, Sphaeroceridae). Acta Entomologica Bohemoslovaca 72: 262-265.

RoháčEK, J. (1998) 3.43. Family Sphaeroceridae. Pp. 463-496. In: PApp, L. \& Darvas, B. (eds): Contributions to a Manual of Palaearctic Diptera. Vol. 3, Higher Brachycera. 880 pp. Science Herald, Budapest.

Roháček, J., Marshall, S. A., Norrbom, A. L., Buck, M., Quiros, D. I., Smith, I. (2001) World Catalog of Sphaeroceridae (Diptera). Slezské zemské museum, Opava, 414 pp.

Su, L. X. \& Liv, G., C. (2009) A new genus and new species of Diptera (Sphaeroceridae, Limosininae) from China. Oriental Insects 43: 49-54. doi 10.1080/00305316.2009.10417575

Vanschuytвroeck, P. 1950. Diptères Sphaeroceridae du Musée du Congo belge. Annales du Musée du Congo Belge, Tervuren, Série in 8 (Sciences Zoologiques) 5: 5-46.

Vanschuтtвroeck, P. 1951. Contribution à l'étude des Sphaeroceridae africains (Diptera Acalyptratae) ( $3^{\text {me }}$ note). Bulletin Institut royal des Sciences naturelles de Belgique, Bruxelles 27(41): 1-20.

Received May 20, 2015, accepted August 27, 2015, published March 16, 2016 